University of Louisville

ThinkIR: The University of Louisville's Institutional Repository

Electronic Theses and Dissertations

$5-2017$

\title{
"Given time..." for soprano and orchestra.
}

Alex Isackson

University of Louisville

Follow this and additional works at: https://ir.library.louisville.edu/etd

Part of the Composition Commons

\section{Recommended Citation}

Isackson, Alex, "'Given time..." for soprano and orchestra." (2017). Electronic Theses and Dissertations. Paper 2645.

https://doi.org/10.18297/etd/2645

This Master's Thesis is brought to you for free and open access by ThinkIR: The University of Louisville's Institutional Repository. It has been accepted for inclusion in Electronic Theses and Dissertations by an authorized administrator of ThinkIR: The University of Louisville's Institutional Repository. This title appears here courtesy of the author, who has retained all other copyrights. For more information, please contact thinkir@louisville.edu. 


\title{
GIVEN TIME...
}

for soprano and orchestra

\author{
By \\ Alex Isackson \\ B.M.A., Brigham Young University Idaho, 2013
}

\begin{abstract}
A Thesis
Submitted to the Faculty of the

School of Music of the University of Louisville in Partial Fulfillment of the Requirements for the Degree of
\end{abstract}

Masters of Music in Music Composition

\author{
School of Music \\ University of Louisville \\ Louisville, Kentucky
}

May 2017 
Copyright ( 2017 by Alex Isackson.

All Rights Reserved. 


\title{
GIVEN TIME...
}

for Soprano and Orchestra

\author{
By \\ Alex Isackson \\ B.M.A., Brigham Young University Idaho, 2013
}

A Thesis Approved on

May 4, 2017

by the following Thesis Committee:

Steve Rouse

Thesis Director

Krzysztof Wołek

Kimcherie Lloyd 


\section{DEDICATION}

To my children,

and to their children, and their children,

and so on...

that their imagination and joy of learning might never

diminish, and that they may always love and appreciate

this beautiful world and wondrous universe

that God has made

for them. 


\section{ACKNOWLEDGEMENTS}

I would foremost like to thank Dr. Steve Rouse for all of his time and expertise that helped me through this project and all the other projects that lead up to it. I'm so grateful for the many helpful hours he spent with me in his office and also at home checking every square inch of my scores. I've learned so much from him and hope to be able to keep learning from him in the future.

Krzysztof Wolek also deserves many thanks for serving on my thesis committee and for his willingness to share his many talents and experiences as a teacher and friend. He has inspired me to do more and be better.

I also want to thank Kimcherie Lloyd for serving on my thesis committee. She has also been very helpful and generous in allowing the university's orchestra to prepare and premier an earlier piece of mine last year. I'm so grateful for all the experiences I've had here at the University of Louisville, and that was certainly one of the highlights.

There have been many other faculty members that have been especially helpful, friendly, inspiring, generous, and knowledgeable and have made an invaluable impact on me here at this university-John Ritz, Jennifer Potochnic, Frederick Speck, Mark Yeary, Mark Dickson, Matthew Ertz, Krista Wallace-Boaz, Caroline Ehman, and Allison Ogden. I enjoyed learning and associating with them.

I need to thank my family and especially my wife, Lindsy, for all the love and support and prayers that helped and encouraged me. They make everything in life better and I'm so grateful for them.

I would also like to thank my poet, Kenny A. Chaffin. Although we have never met, he was so willing to let me use his inspiring and thought-provoking poems as the text for this work. I set out looking for literature about the cosmos and the endlessness of time and eternity. Of the countless poems I read, Chaffin's were exactly what I hoped to find and I was blessed that he was kind enough to let me use them. It was my goal to bring out the wonderment I felt as I read these beautiful poems. 


\section{TABLE OF CONTENTS}

Page

(n)......ii

Acknowledgments

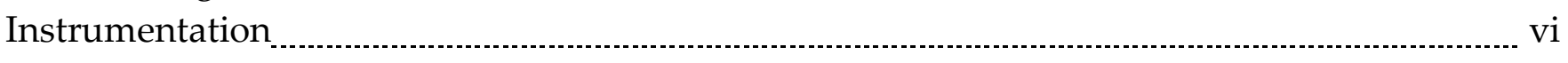

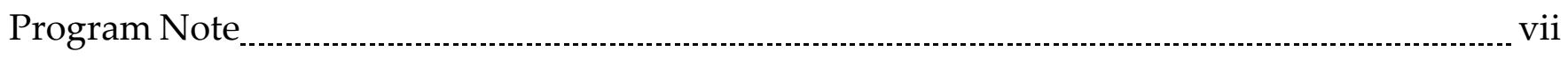

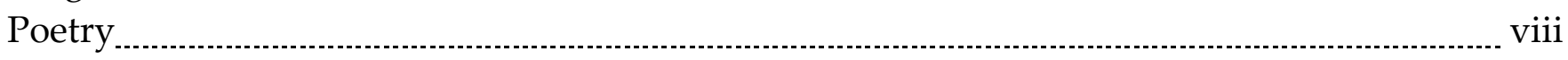

Given time..

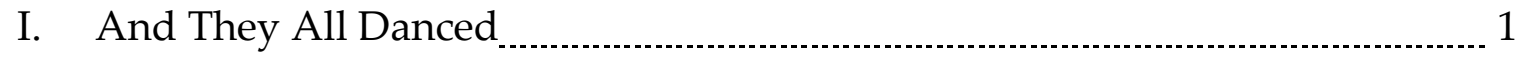

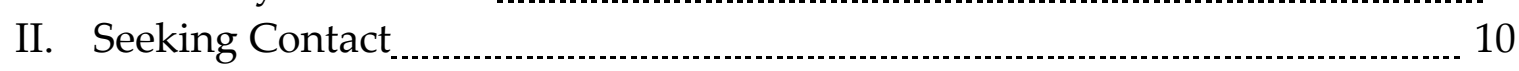

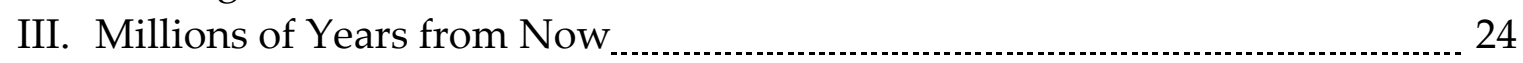

Curriculum Vitae 


\section{INSTRUMENTATION}

Flute 1-3 (2nd doubling Alto and Bass Flute, 3rd doubling Bass Flute and Piccolo) Oboe 1-3 (3rd doubling English Horn)

Bb Clarinet 1-2

Bb Bass Clarinet

Bassoon 1-2

Contrabassoon

F Horn 1-4

C Trumpet 1-3

Trombone 1-2

Bass Trombone

Tuba

Timpani

Percussion 1: Tam-tam, Suspended Cymbal, Bass Drum, Tubular Bells (shared), Marimba (shared), Temple Blocks, Sizzle Cymbal

Percussion 2: Glockenspiel, Tubular Bells

Percussion 3: Vibraphone, Marimba

Harp

Piano

Solo Soprano

Strings (Minimum: 8, 7, 6, 5, 4) 


\section{PROGRAM NOTE}

The title of this work, Given time... is meant to be less of a title and more like the beginning of a deep thought. Time is one of those things that I think humans just don't understand and don't give very much thought to, especially considering how much time has already passed and how much time left there is in the universe. Where does space and time end? And where do we (or I) fit in all of it? Does everything I do have any lasting impact on the eternal scheme of things? The list of questions goes on seemingly forever, but pondering them has always been stirring to me. Setting some of these thoughts and feelings to music was a new challenge that I loved every step of the way.

This work sets three poems by Kenny A. Chaffin. Several more of his poems were selected to be included in the work at a later date. Each poem introduces a new way of looking at or thinking about the vastness of the universe, the endlessness of time, and in some cases, our potential impact or fate as a human race.

"And They All Danced" is about an incomprehensibly slow dance that has gone on for billions of years and will continue to go on forever. It is the dance of the galaxies as they move, twirl around, and sometimes collide with each other. The music is slow but filled with anxious anticipation. It is meant to give the impression of something small and docile, yet enormous and powerful at the same time-just like the drifting galaxies through space. There are a couple moments in the music where I imagined an extremely slow "downbeat" as if we were listening to the dance music of the universe slowed down by a thousand times - or is it sped up?

"Seeking Contact" comes from mankind's current perspective and relationship with the universe. For decades now we have been probing the galaxy and listening for any kind of a response. It reflects our innate desire to answer the questionare we alone in the universe? As Arthur C. Clarke famously said, "both [answers] are equally terrifying." The music reflects our persistent effort to reach out in search of life beyond this planet and possibly a hint of apprehension at finding out the answer.

"Millions of Years from Now" is an attempt to reflect the thoughts of someone living millions of years from now as they remember their solar system of origin. After having populated the rest of the Milky Way and beyond, would we look back with fondness or disdain? I imagine the speaker of this poem as a spoiled and distracted twenty-something living millions of years in the future who's decided that the new, cool, retro thing is the Oort Cloud-a spherical shell of icy objects that exist in the outermost reaches of our solar system. 


\section{POETRY}

by Kenny A. Chaffin

\section{And all the Galaxies Danced}

It was a slow dance

slower than anything

comprehensible by

mere mortal humans.

M81 and 82 twirl

like lovers enthralled.

Dancing, merging, twirling about

under billions of brilliant stars.

The Milky Way dips

Andromeda and swings

her out and away, before

pulling her tight again.

The dance goes on above our heads though we never see,

galaxies dancing for billions of years to a lilting cosmic beat.

\section{Seeking Contact}

Like gossamer threads reaching out we seek the stars

Driven to know, to grow, to search we push ever outward

No matter the quantum odds

no matter the impossible distances

We seek others of our kind, in our image reflected in the stars

\section{Billions of Years from Now}

The Oort Cloud now is cool again not in the sense of temperature though surely it's cooled a bit

It's cool because after billions of years of spreading out seed over distant galaxies and times

we've turned our attention with a sense of nostalgia to the place where we were born. 


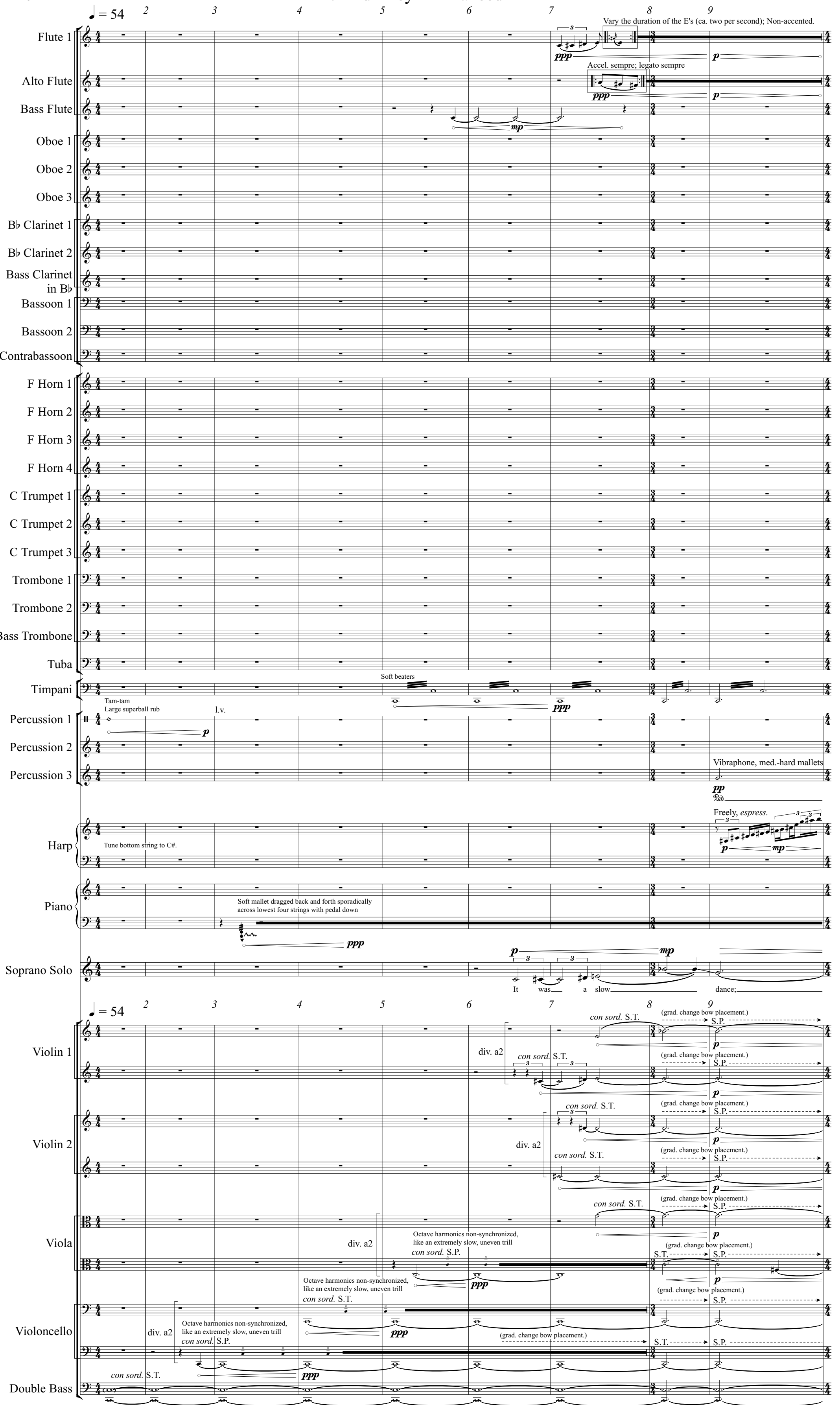



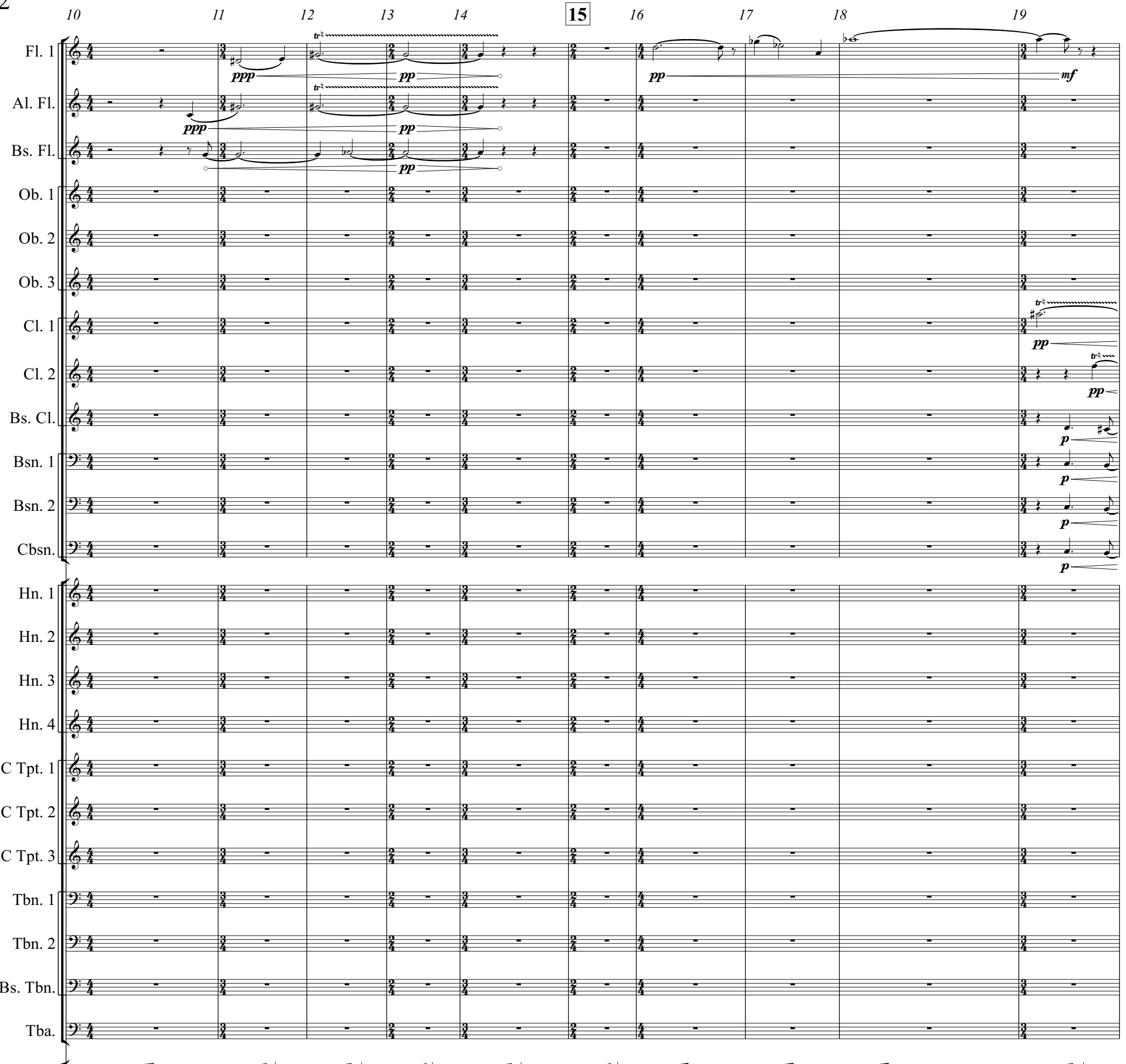

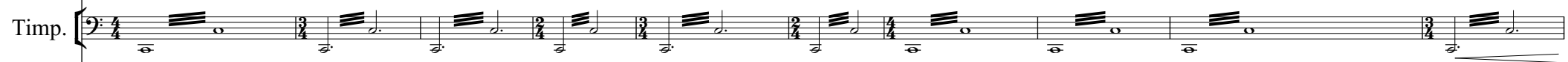

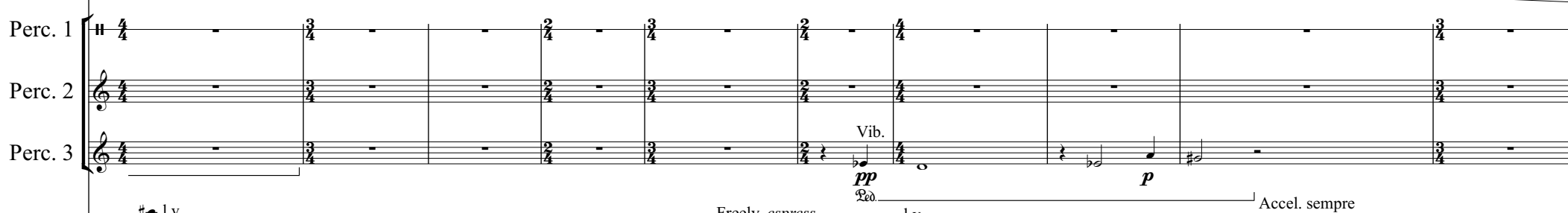

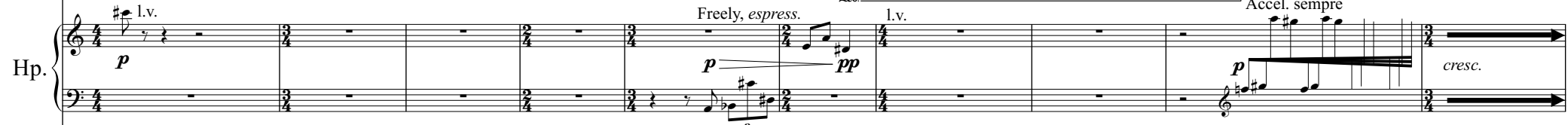

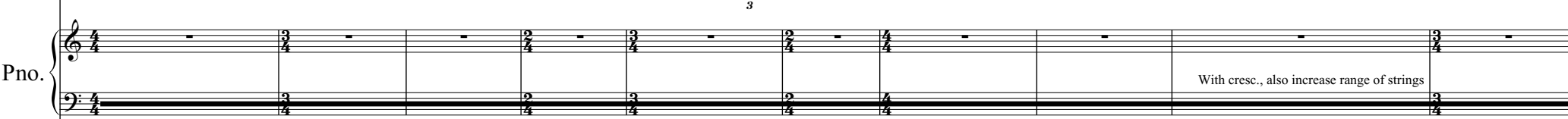

S. Solo $\mathrm{b}_{4}$

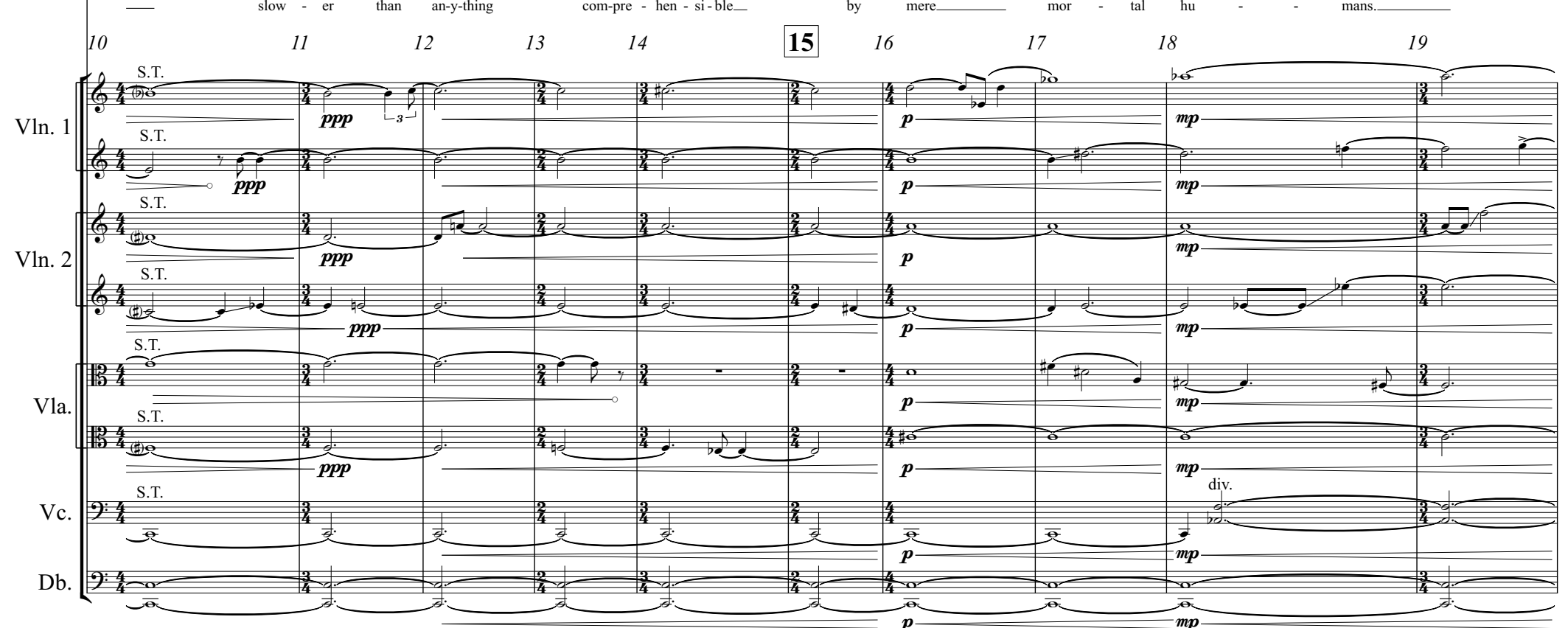




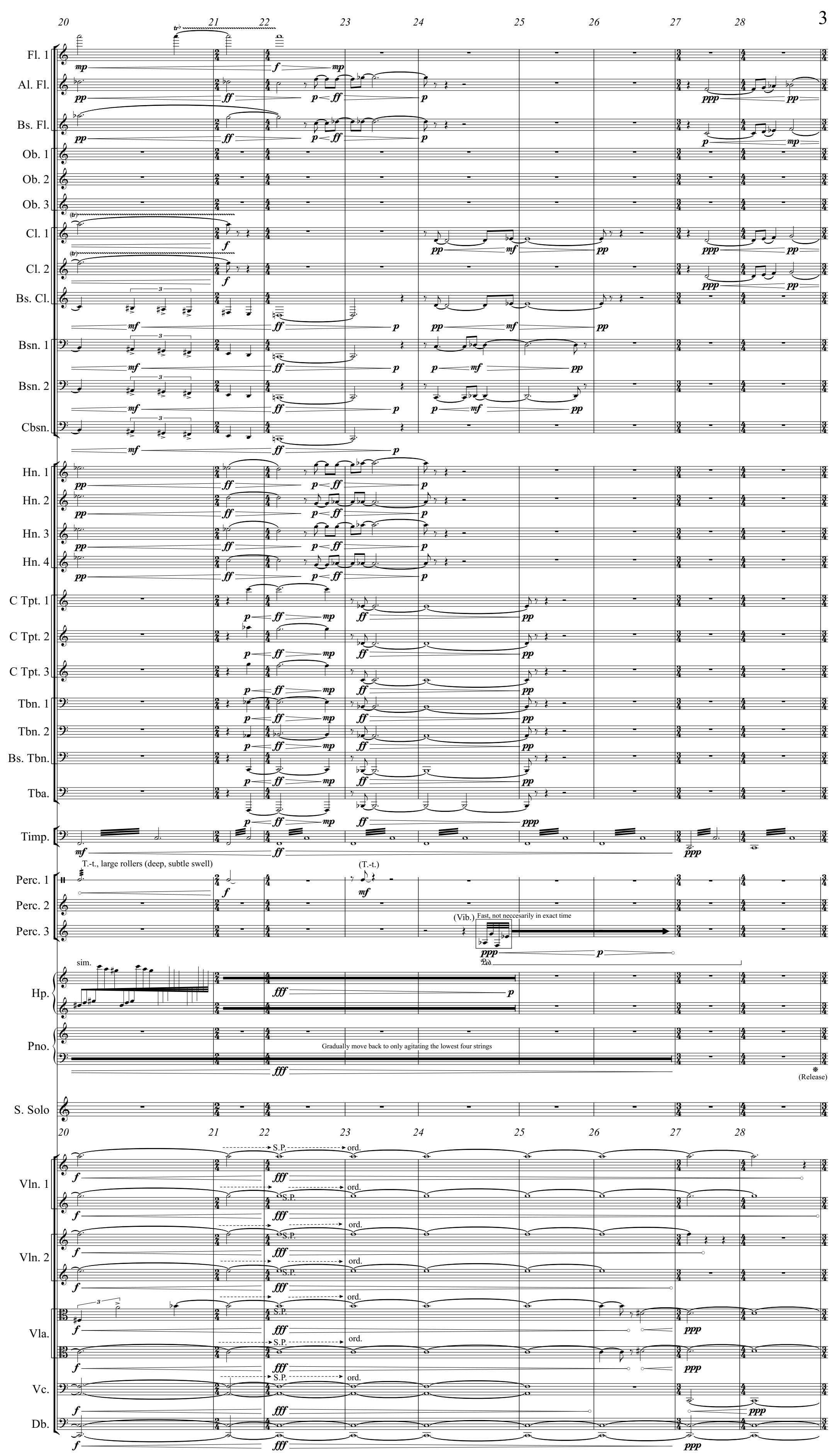


$29 \quad 30$

31

32
Vary the duration of

33
33
jer second); Non-accented.

$34 \quad 35$

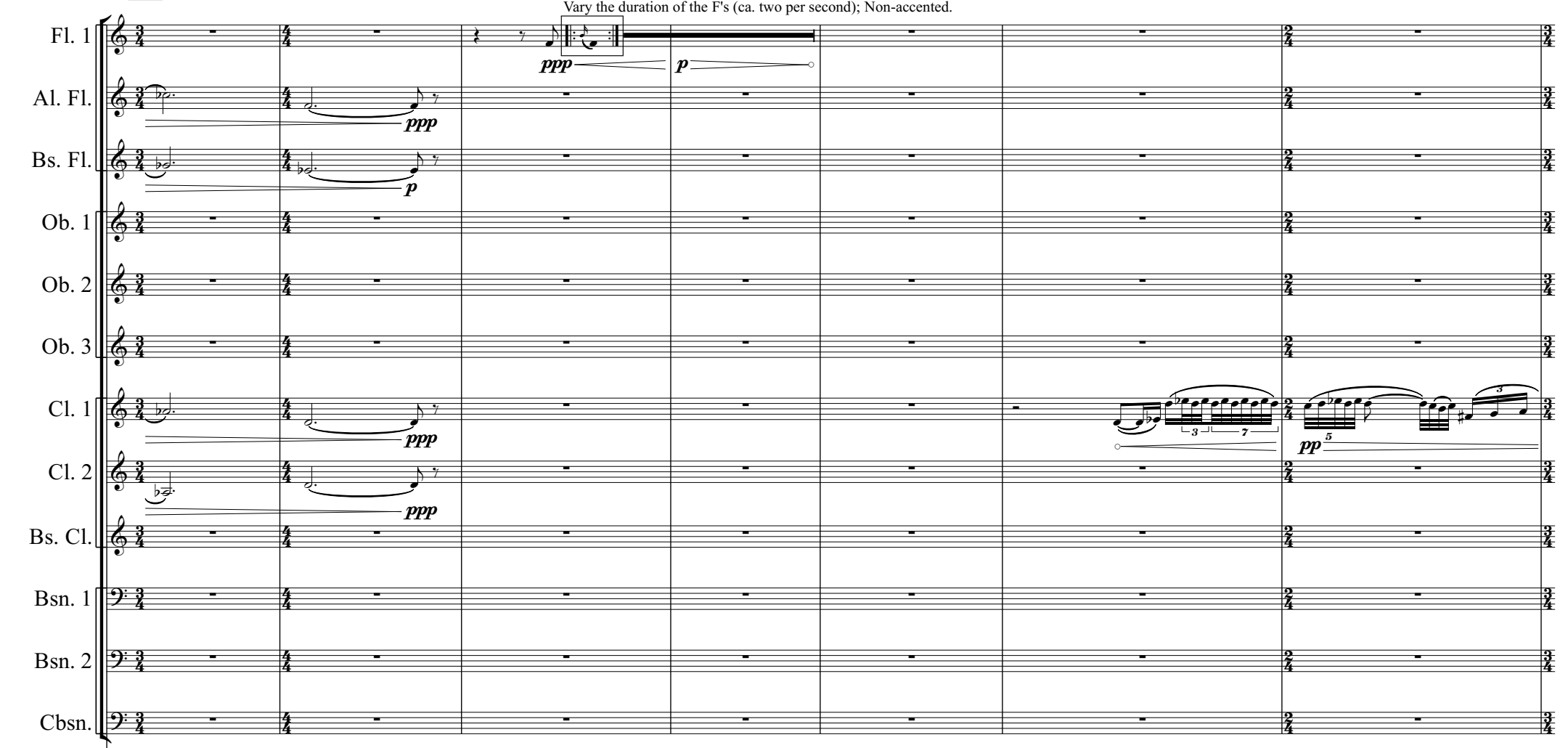

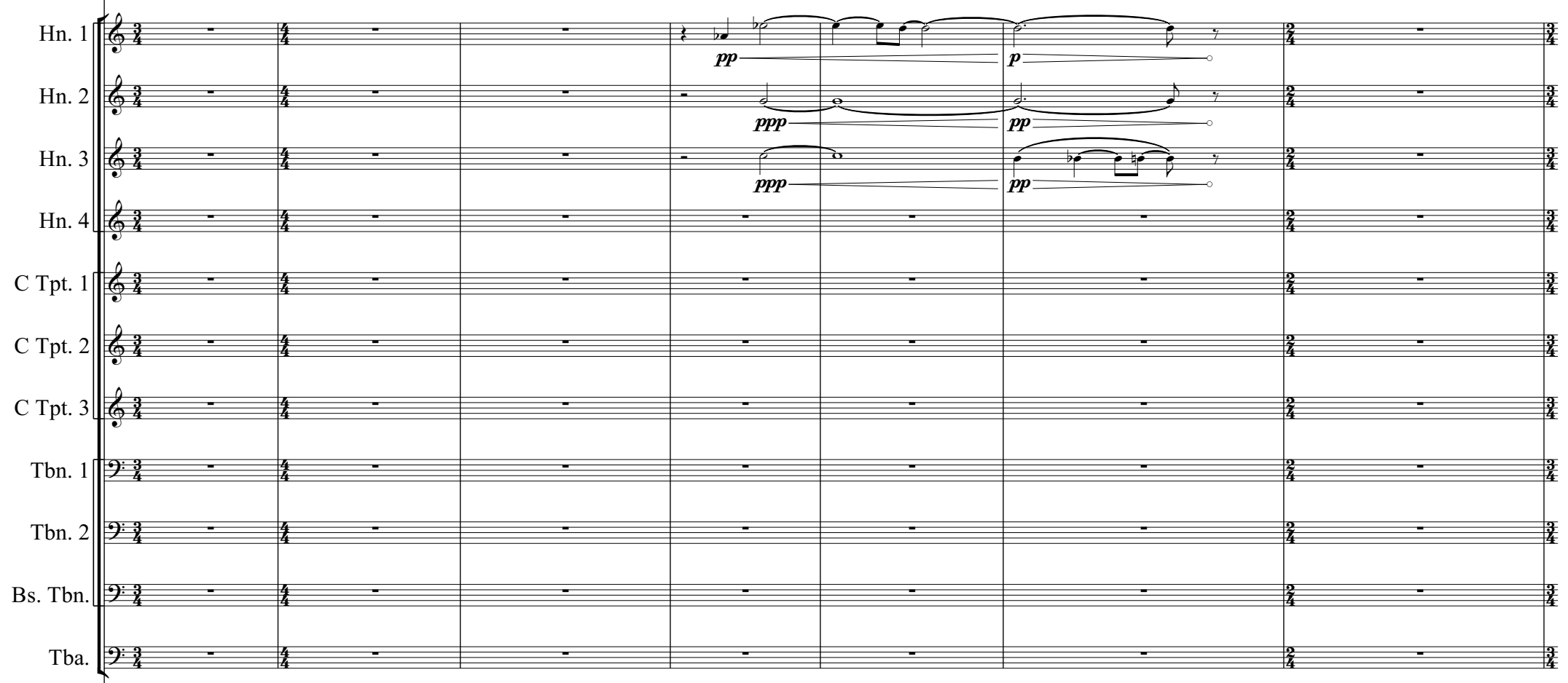

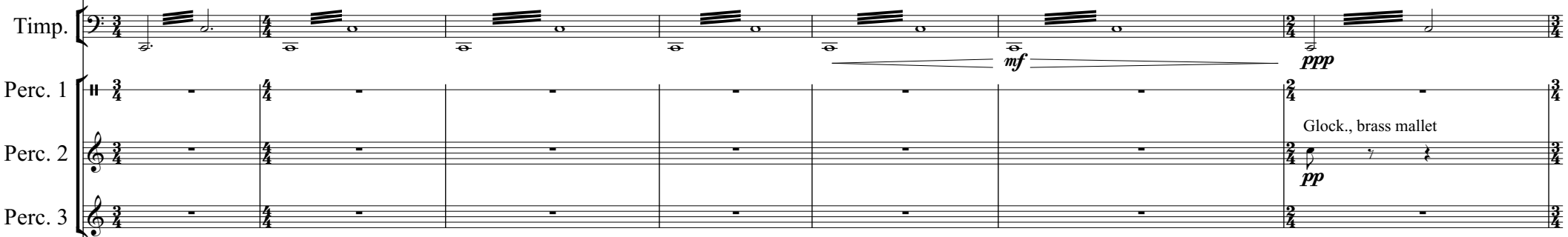

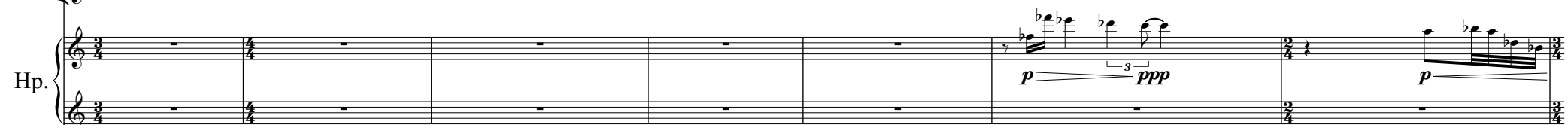

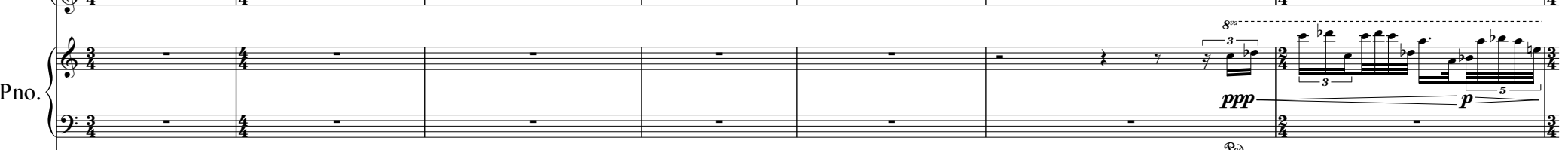

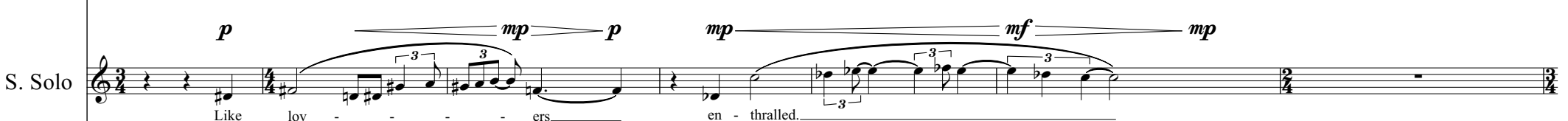

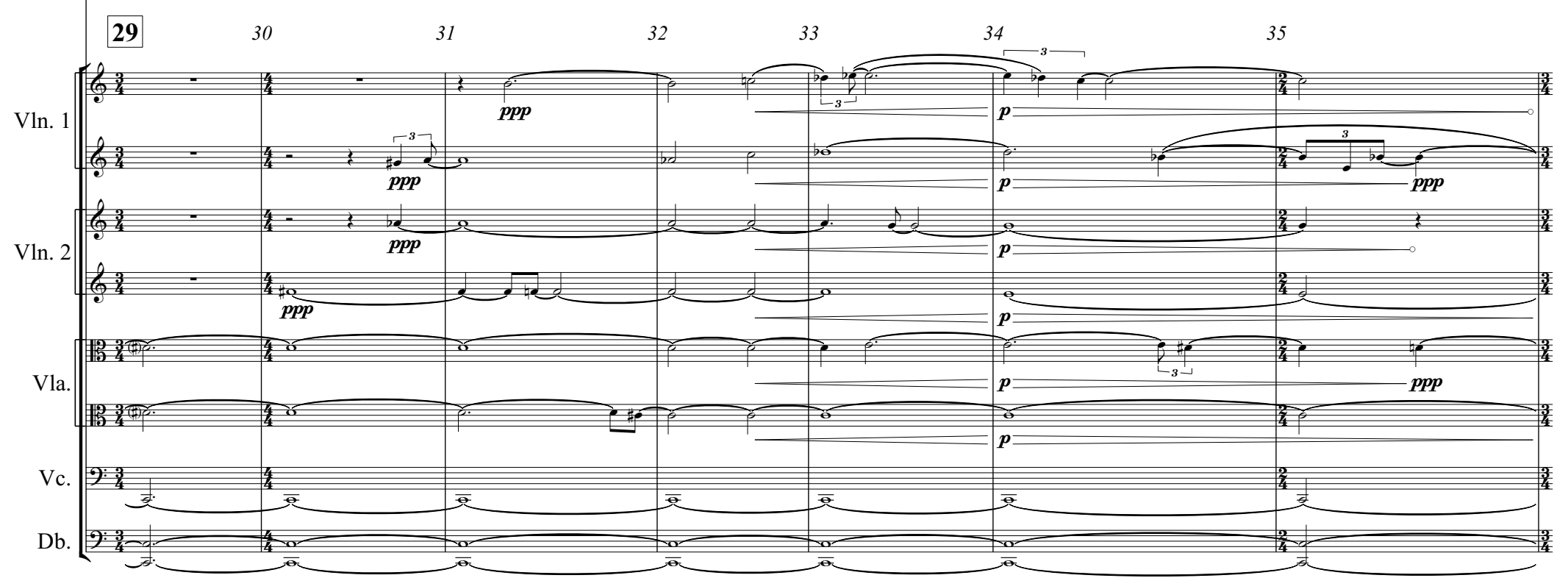




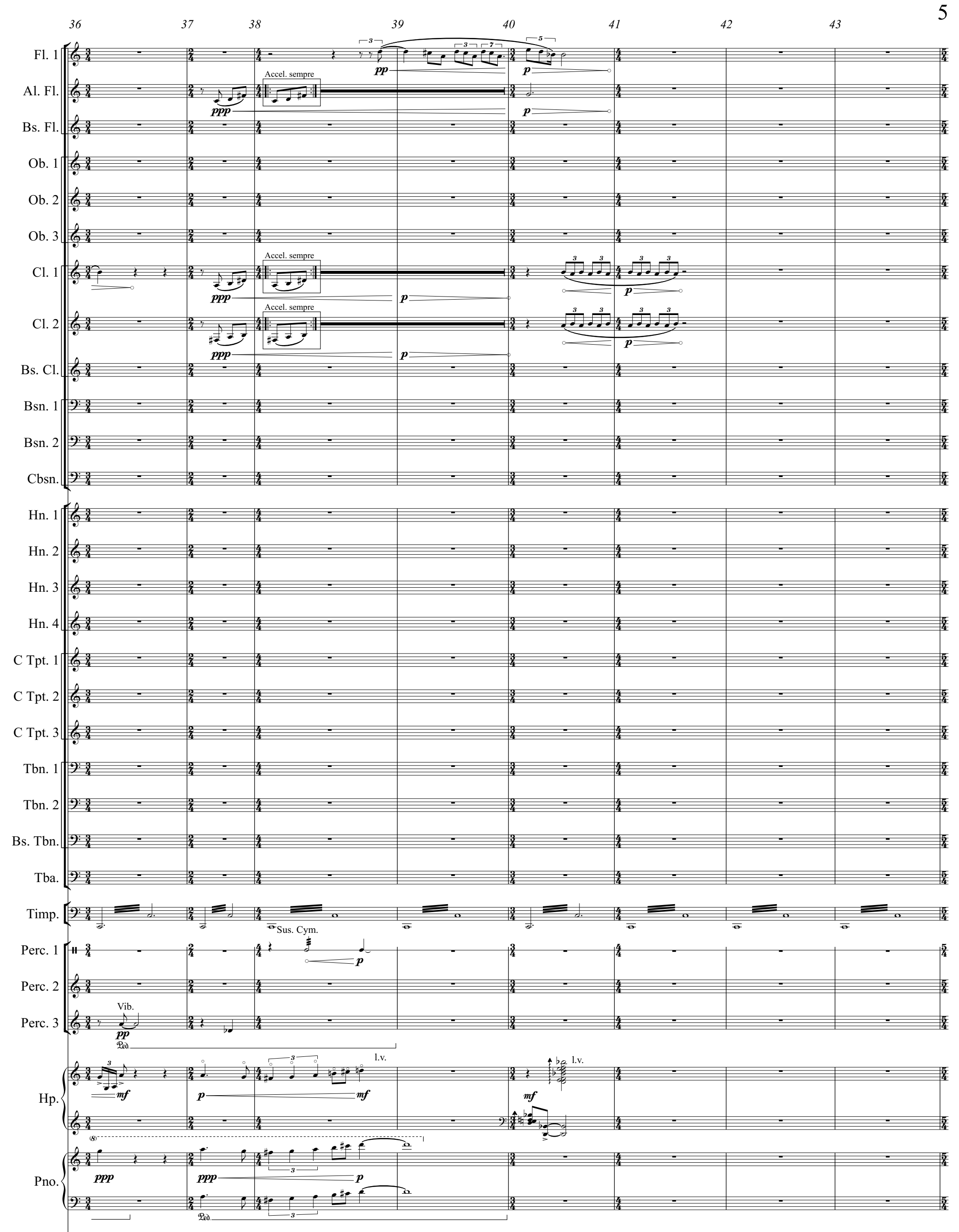

S. Solo

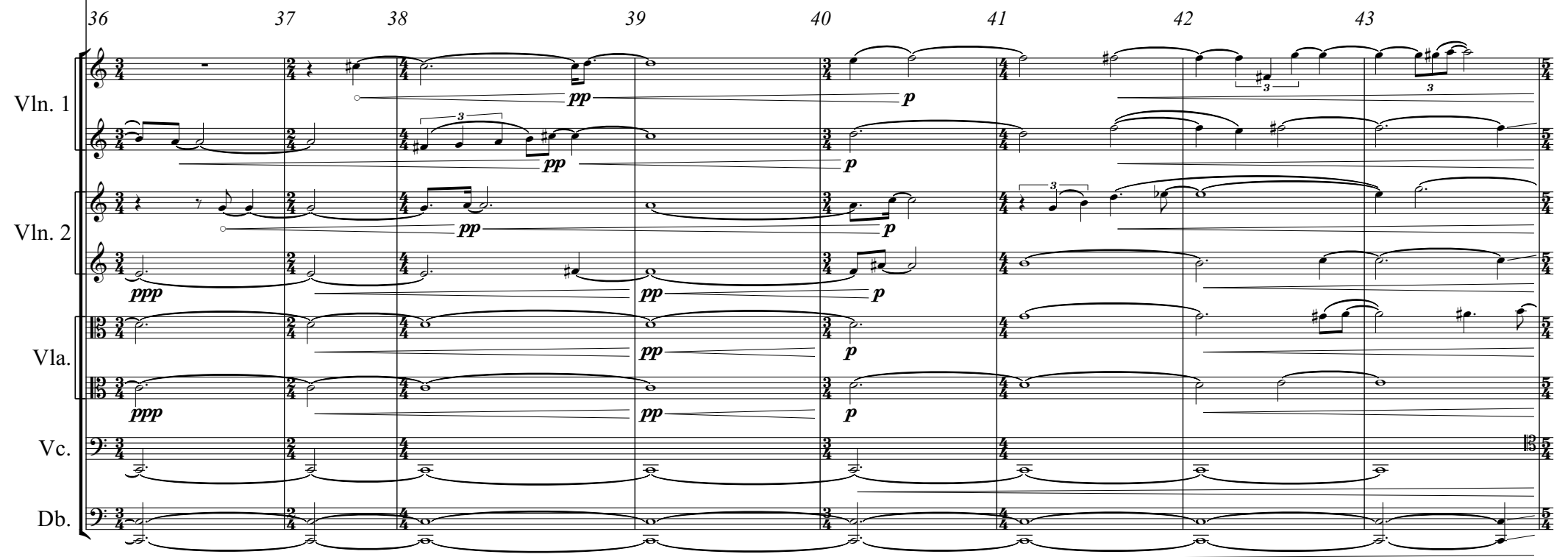



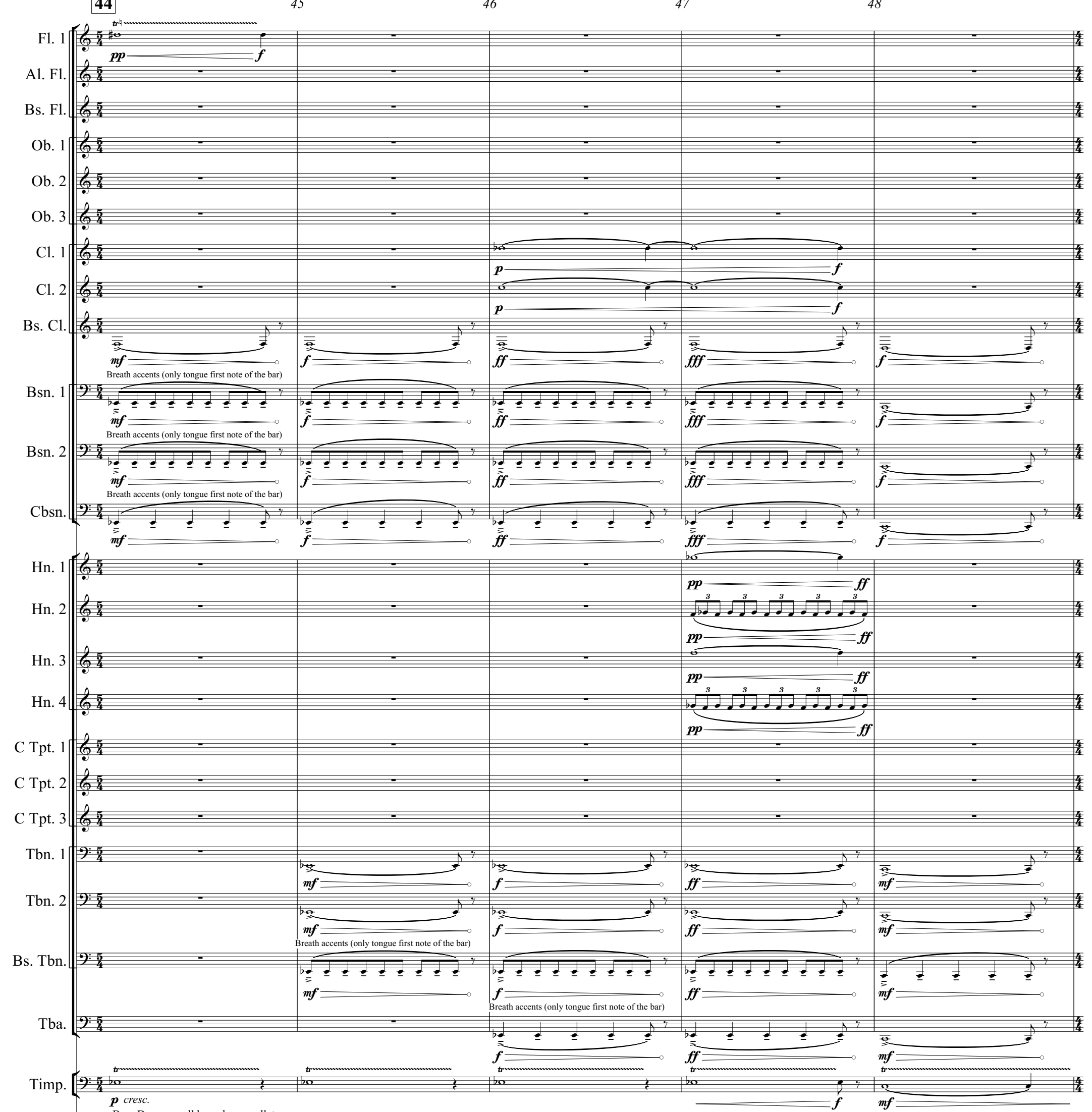

Timp.

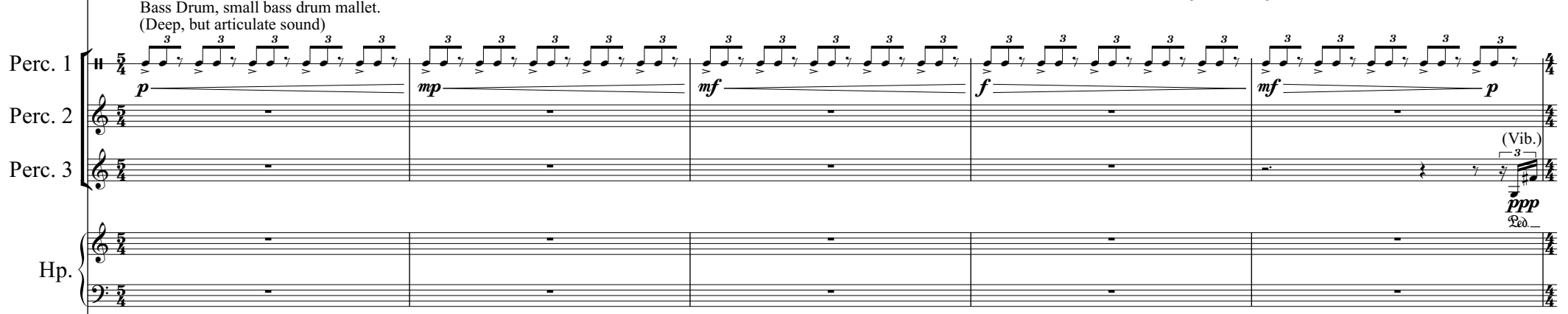

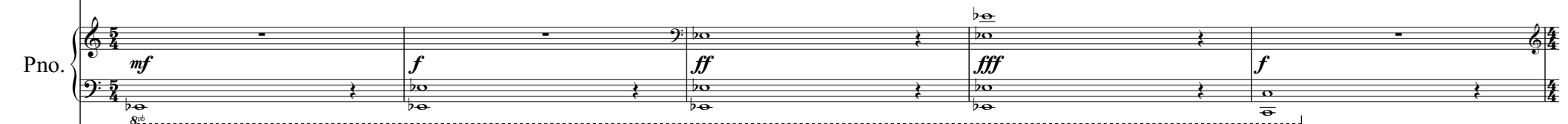
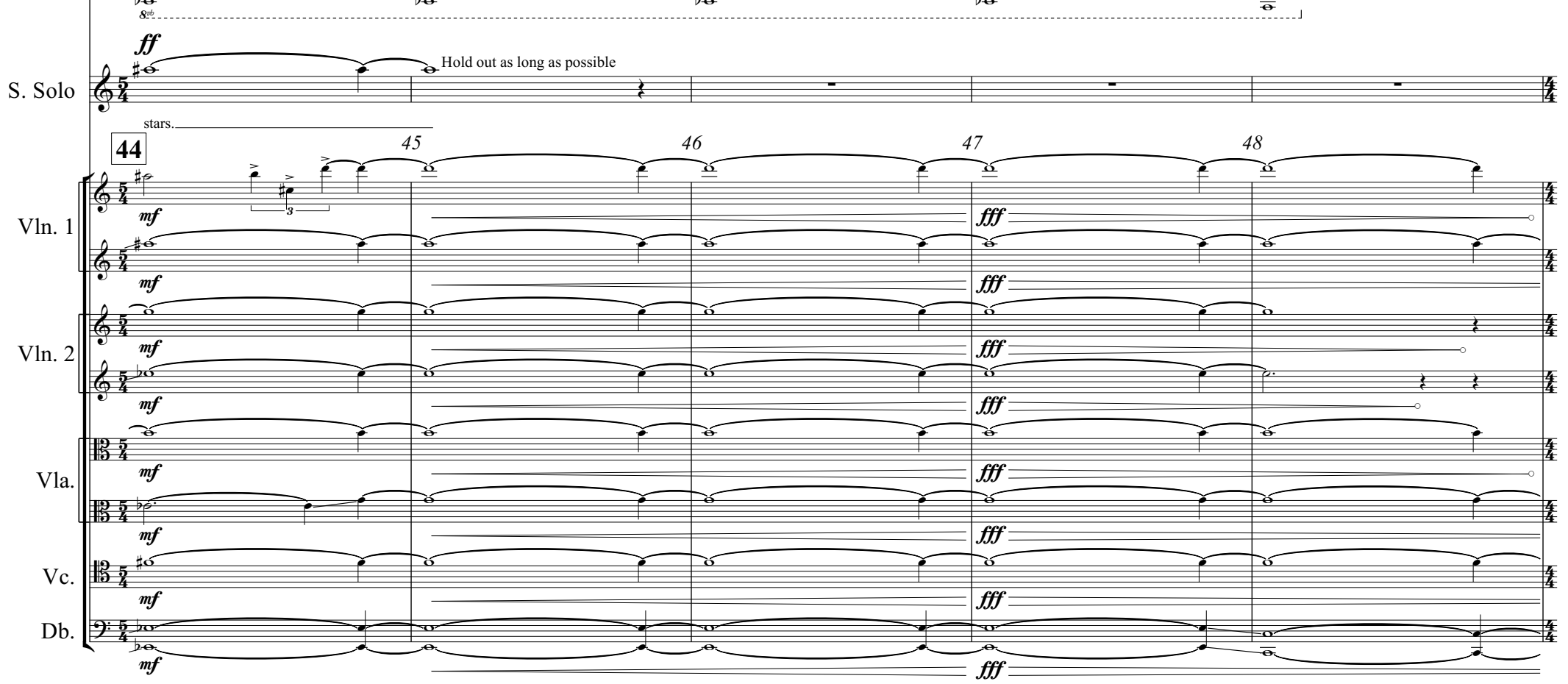


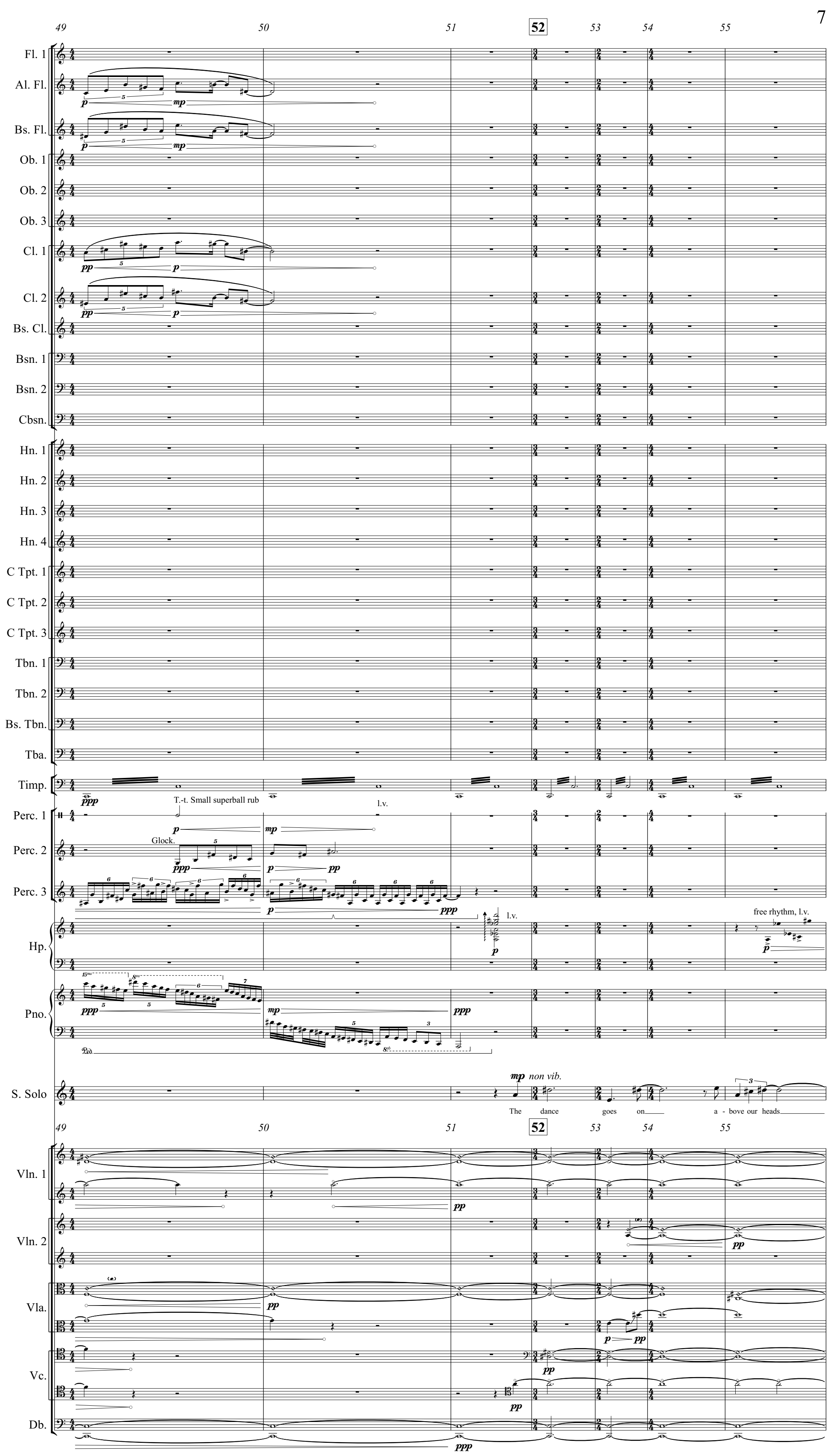



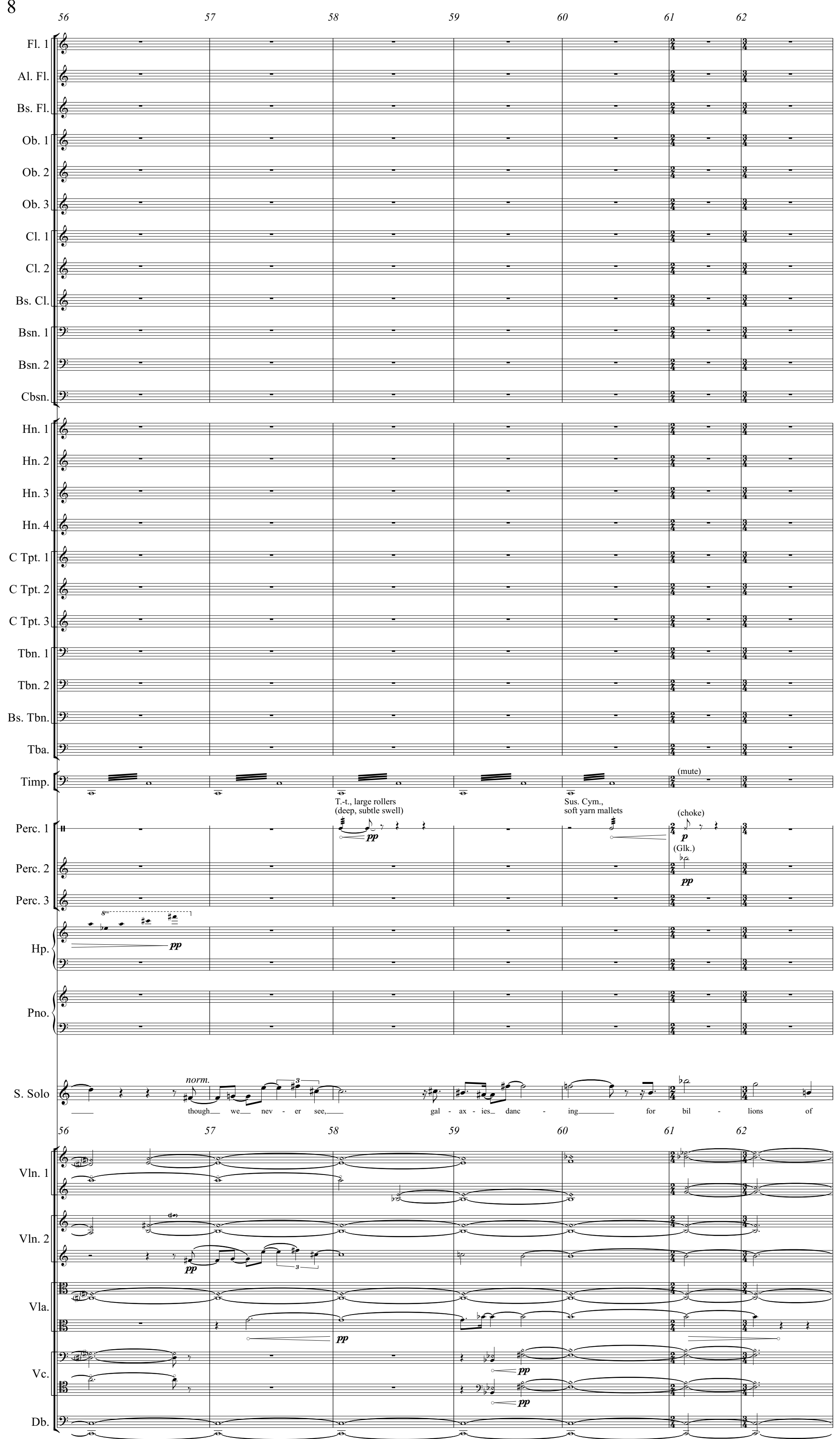


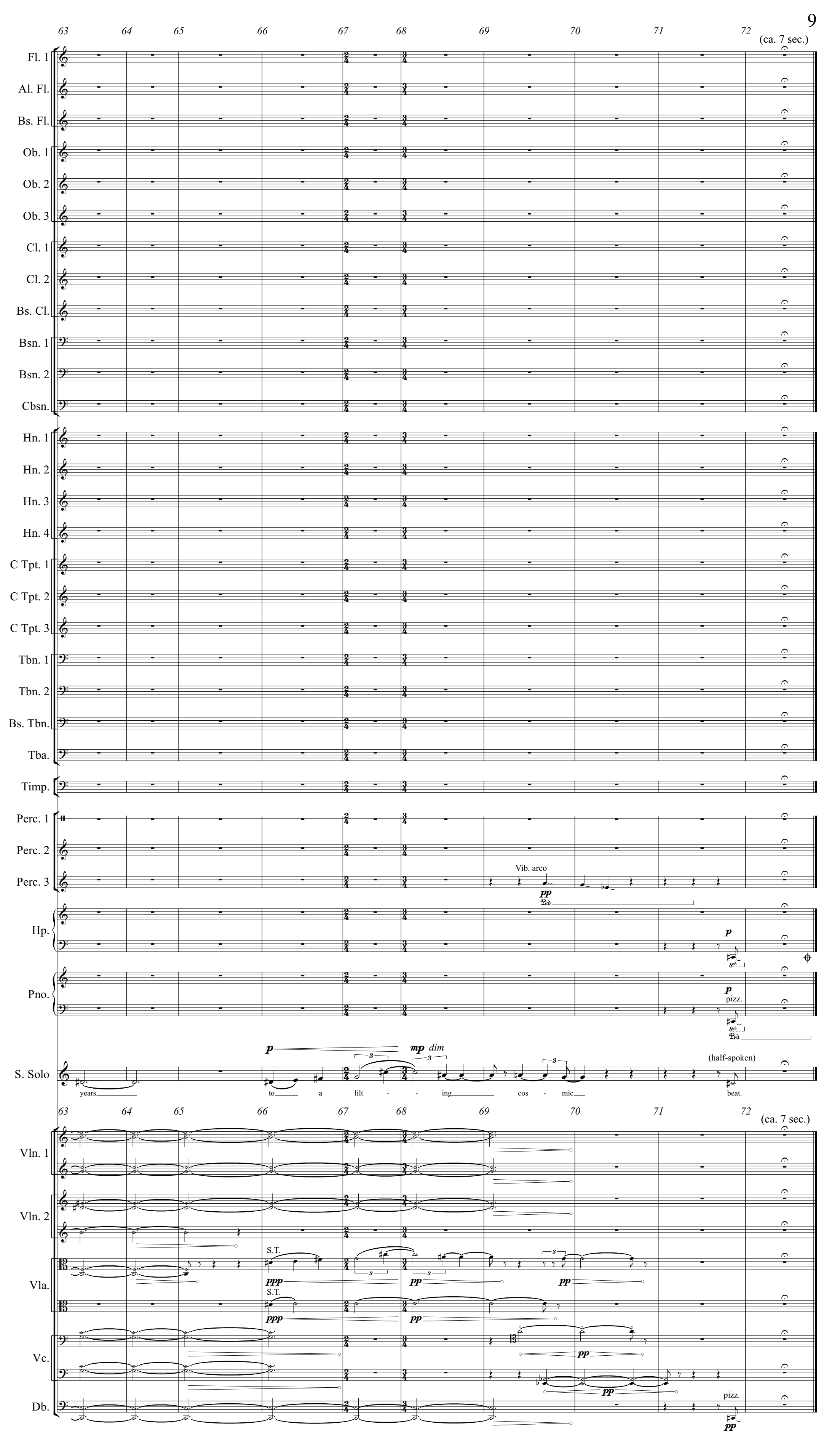



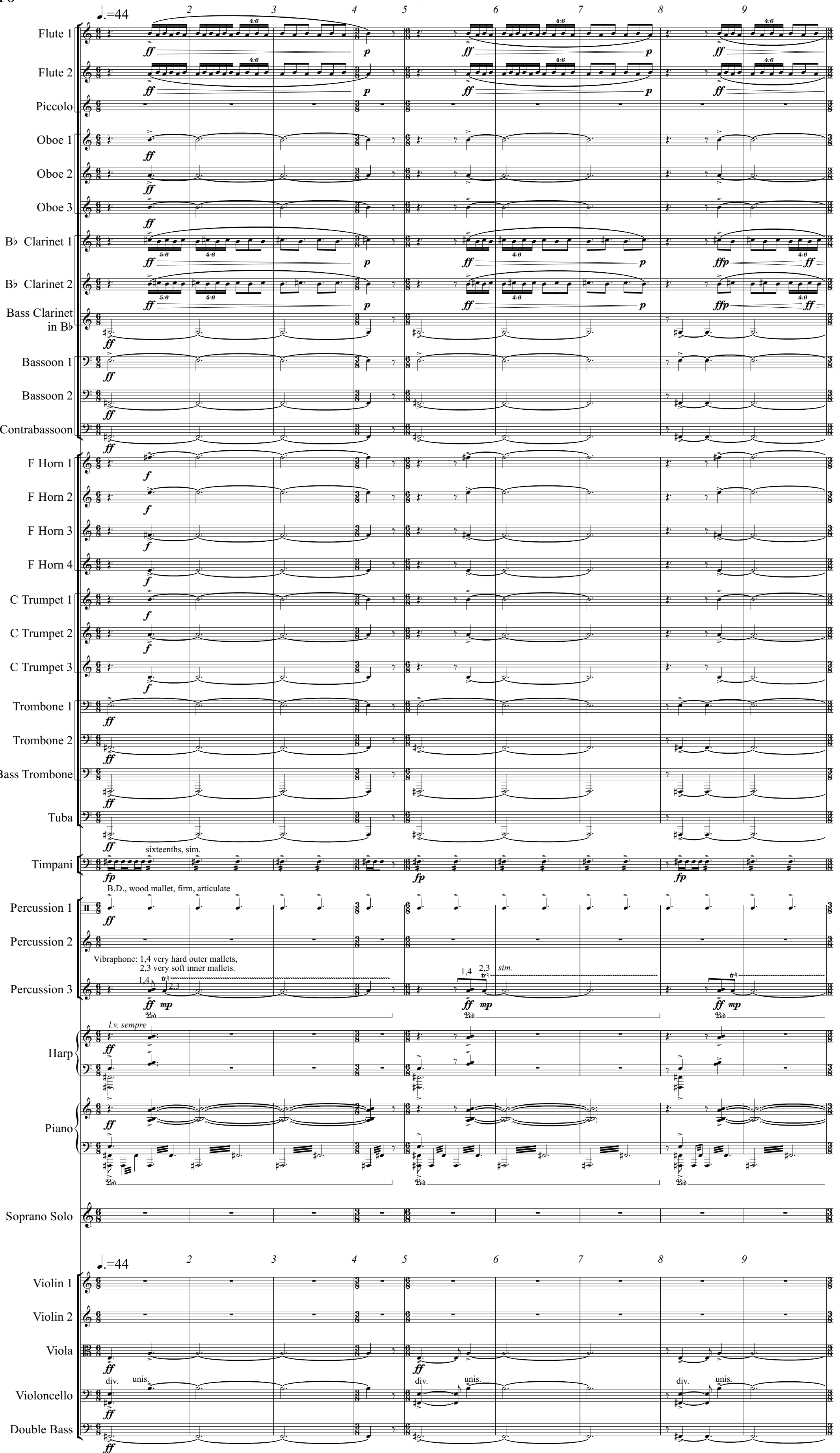


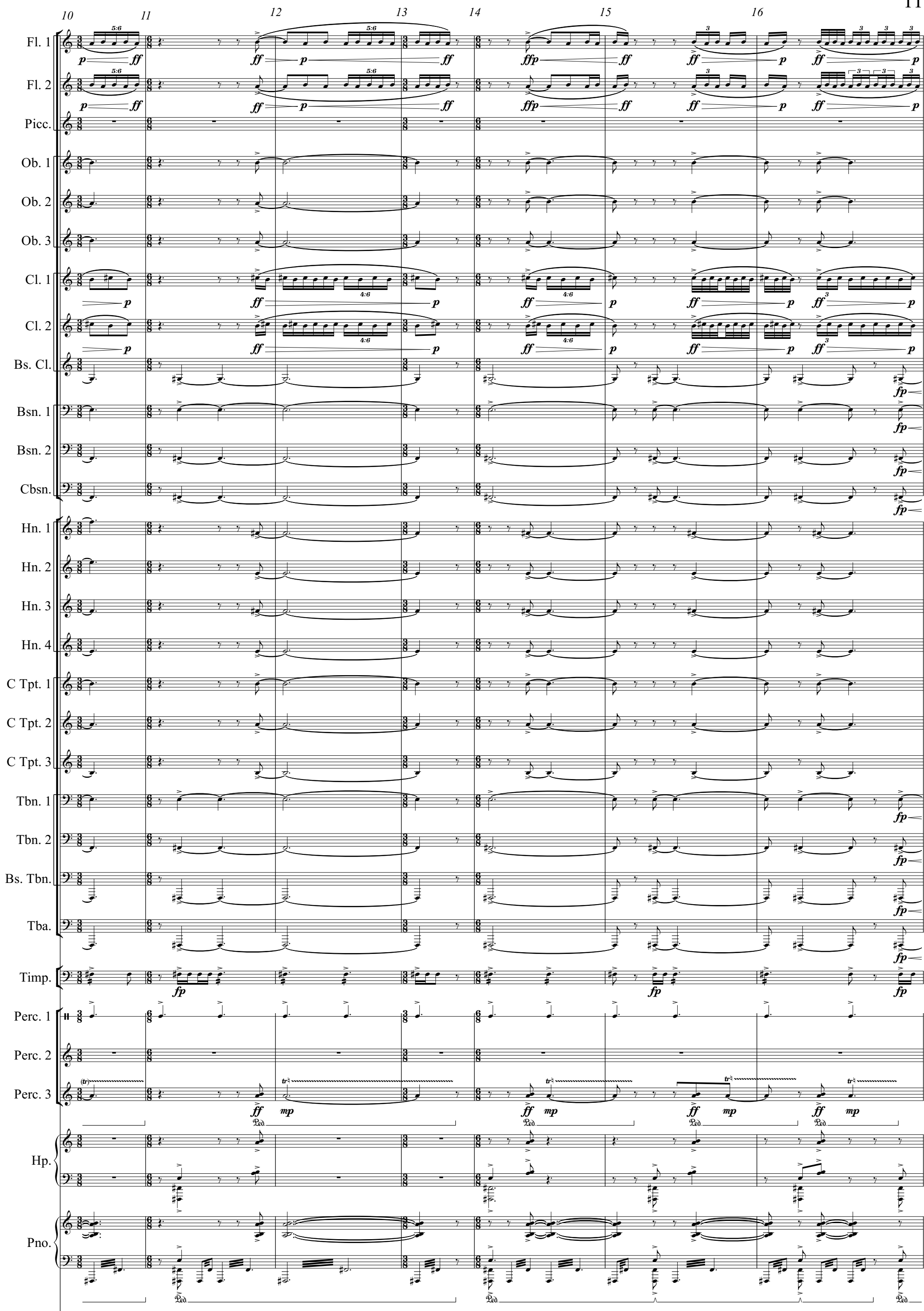

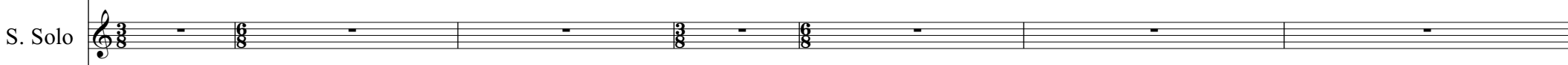

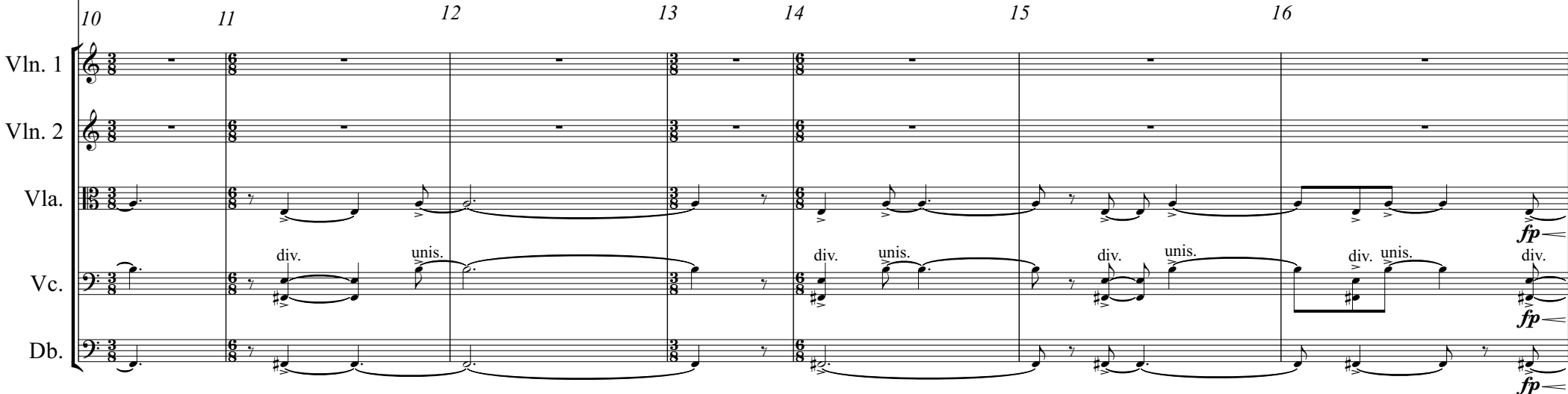



18

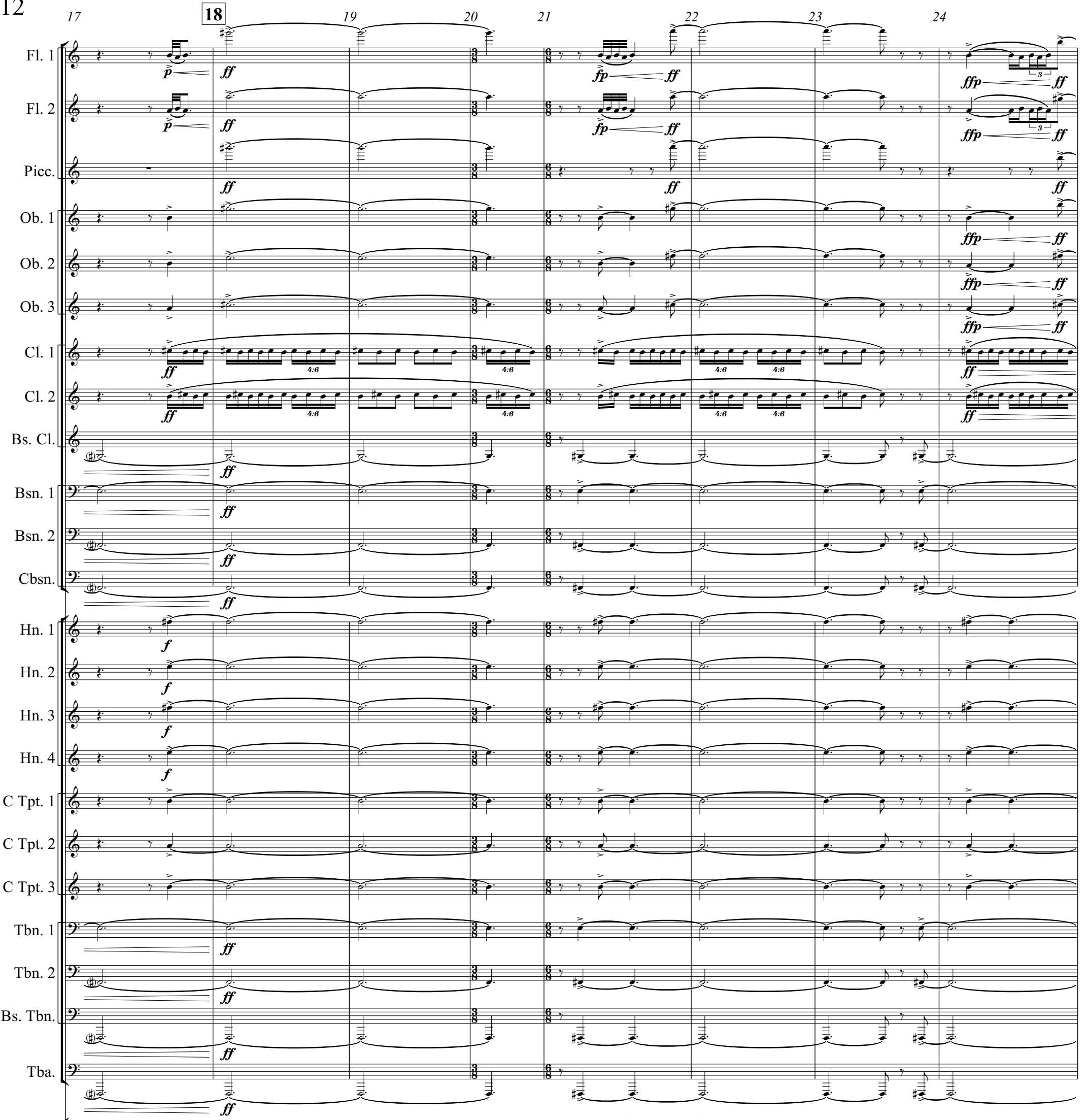
Timp.

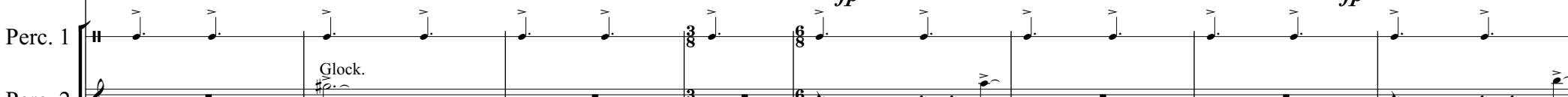
Perc. 2 Perc. 3 (b)

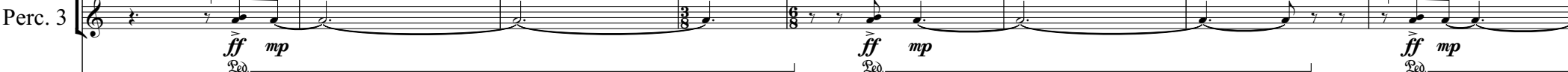

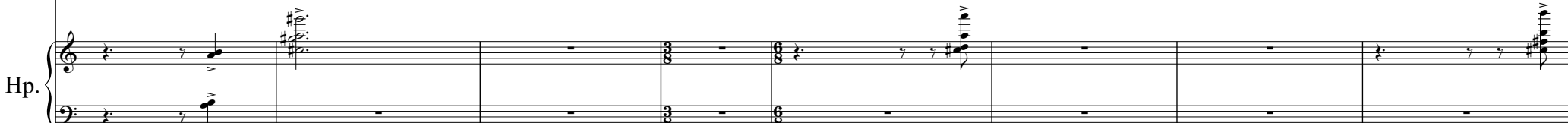

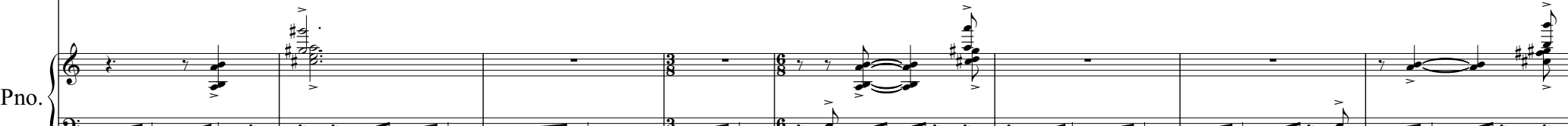

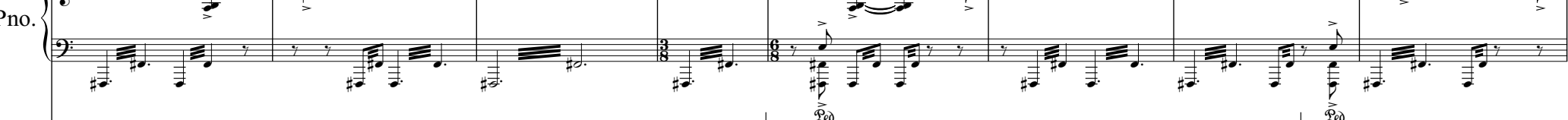
S. Solo $\frac{1}{3}$ Vln.2 


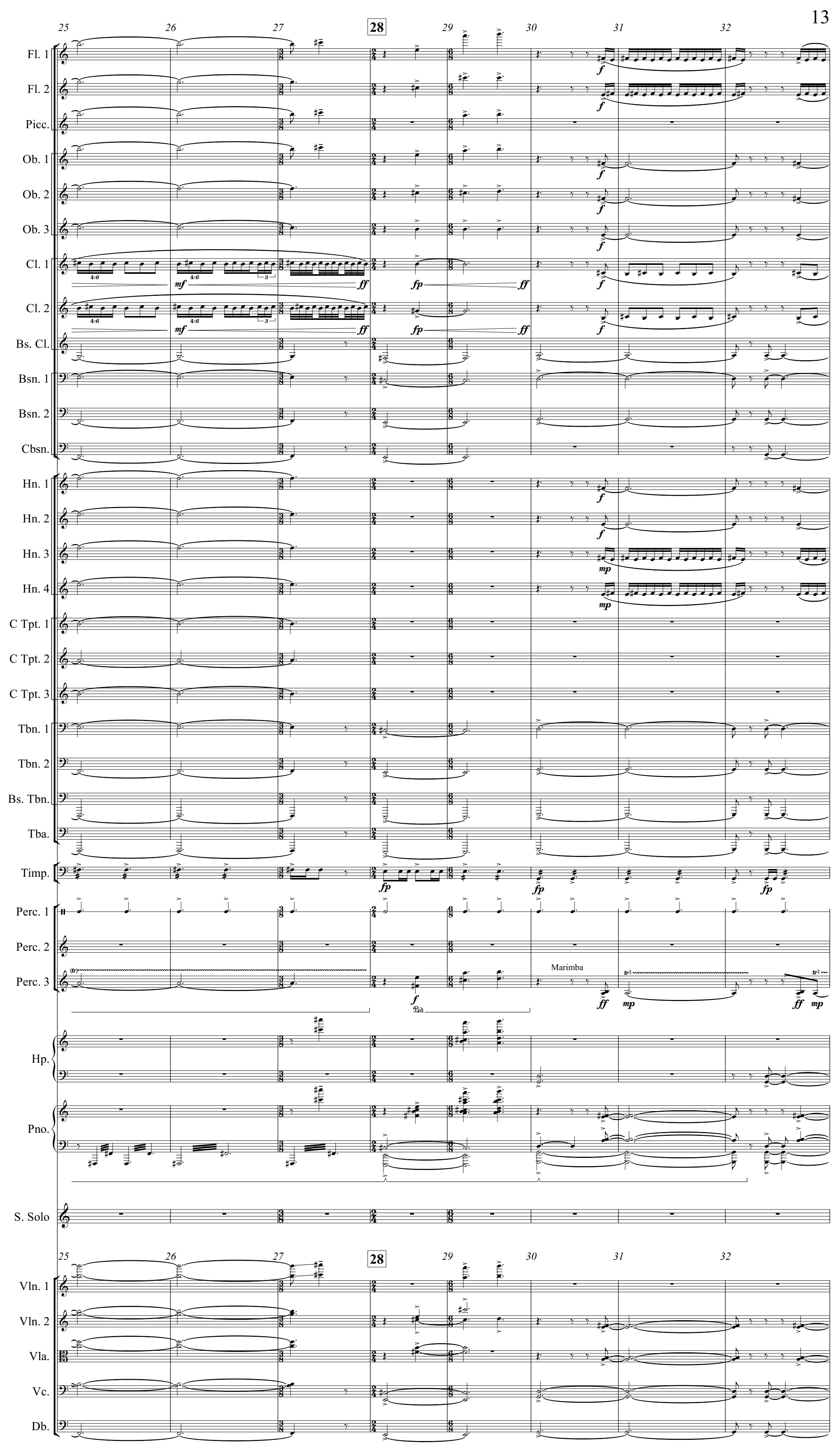


accel.

36

38 .. $=54$

40

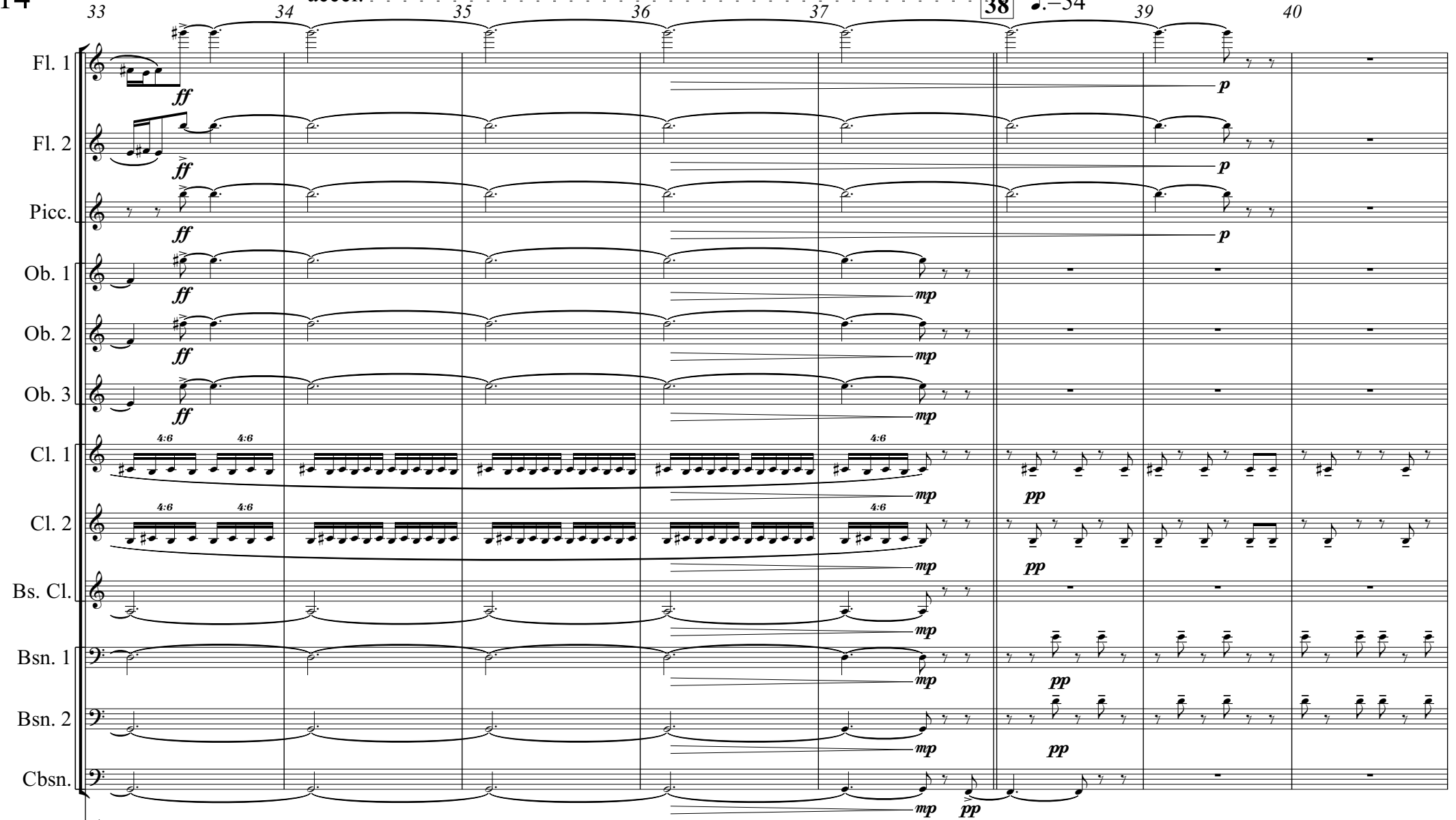

Bs. Tbn.

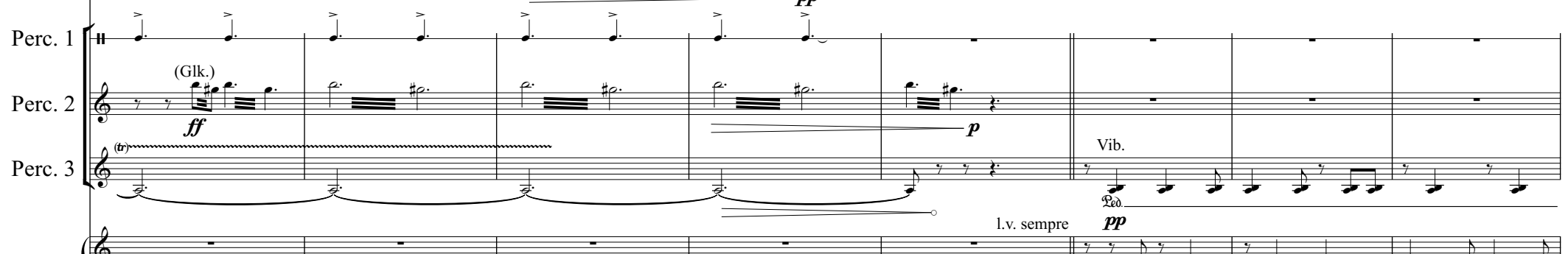

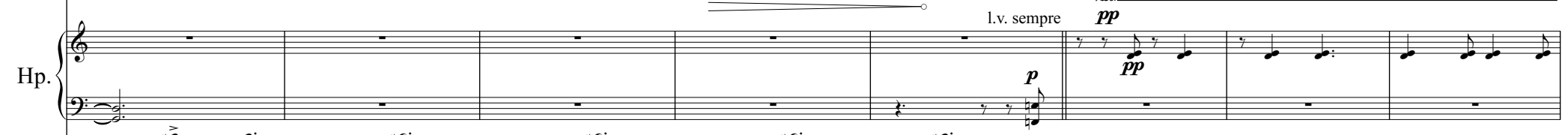

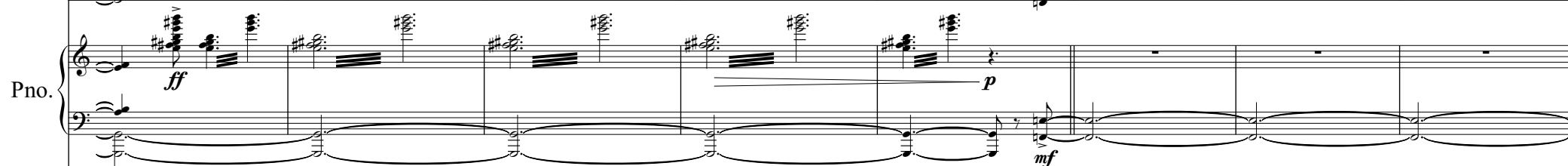

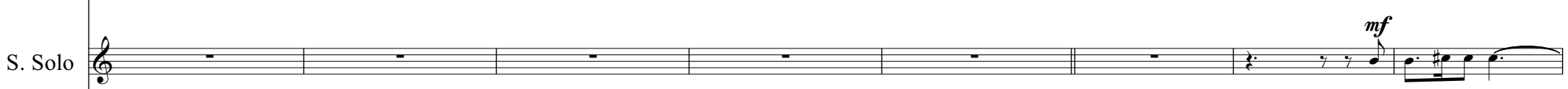

Vln. 2 Vla. 1 VIa 


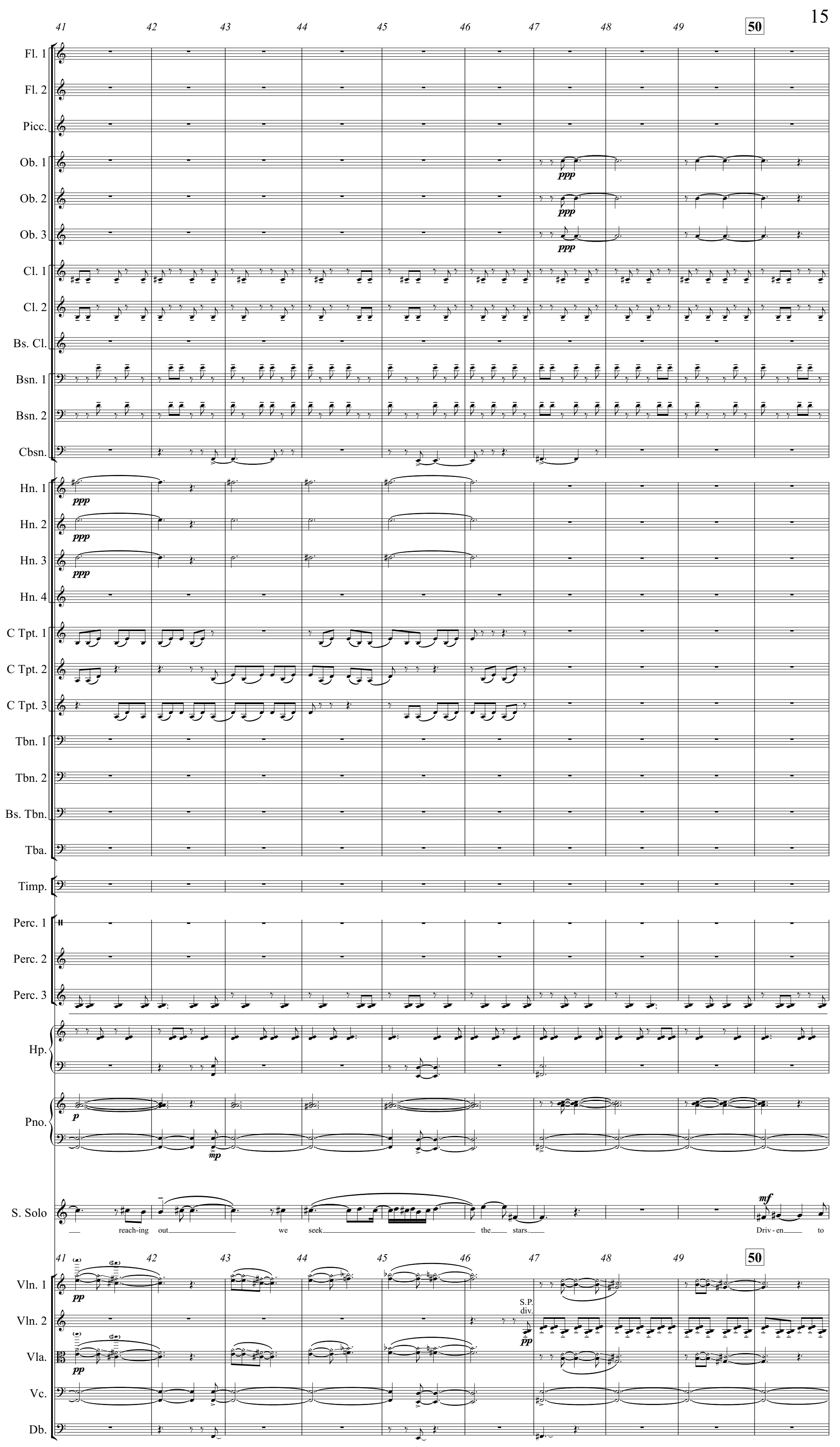




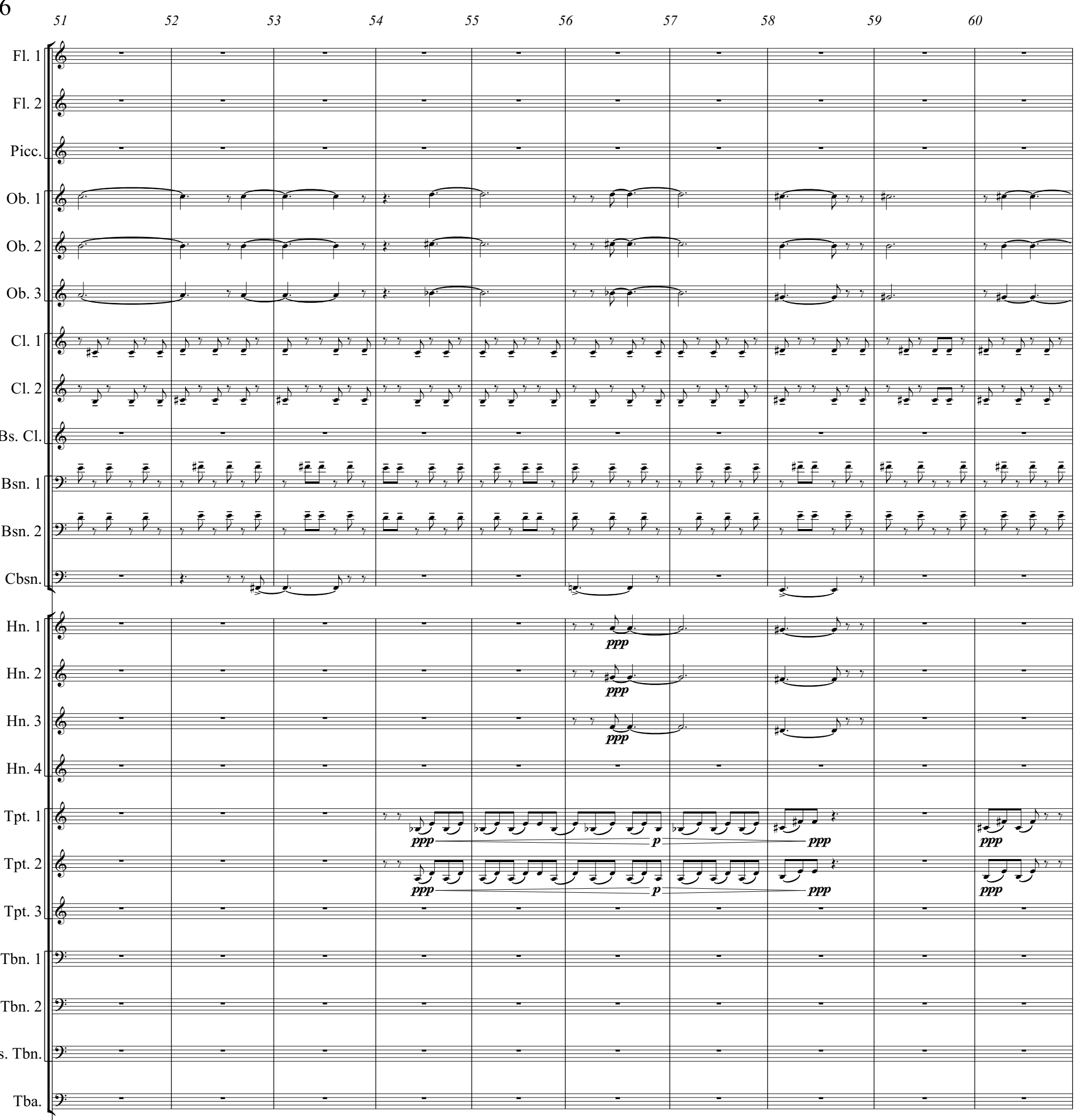

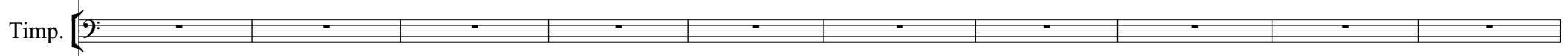

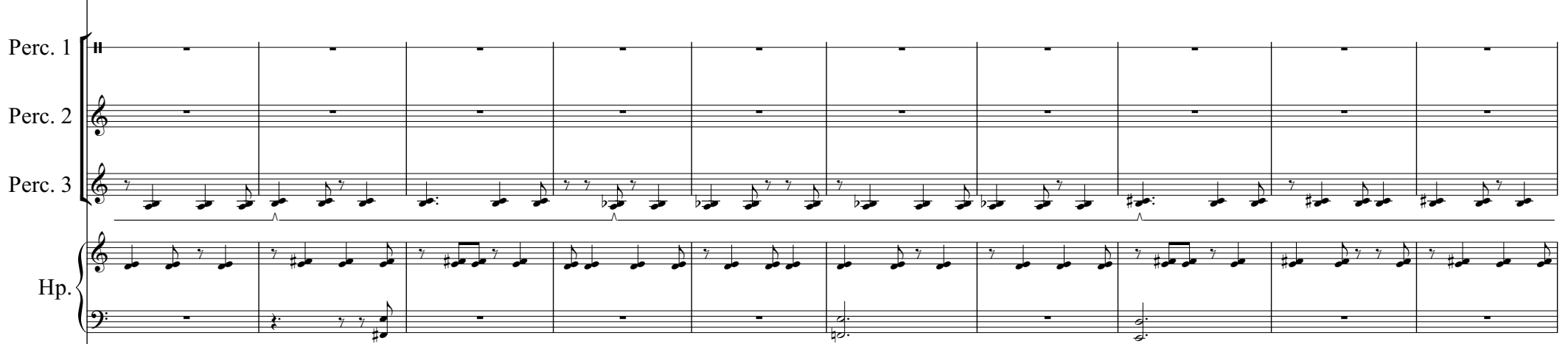

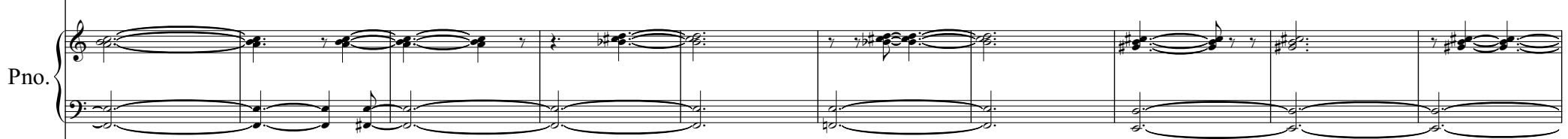

S. Solo $\sum_{\text {know, }-}^{0}$ to grow, $\begin{array}{llllllllll}51 & 52 & 53 & 54 & 55 & 56 & 57 & 58 & 59 & 60\end{array}$

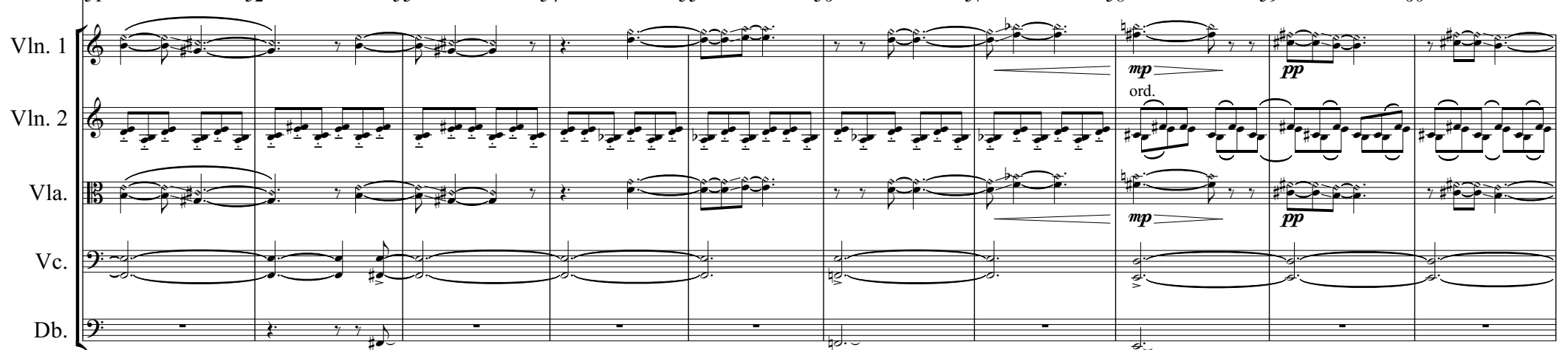




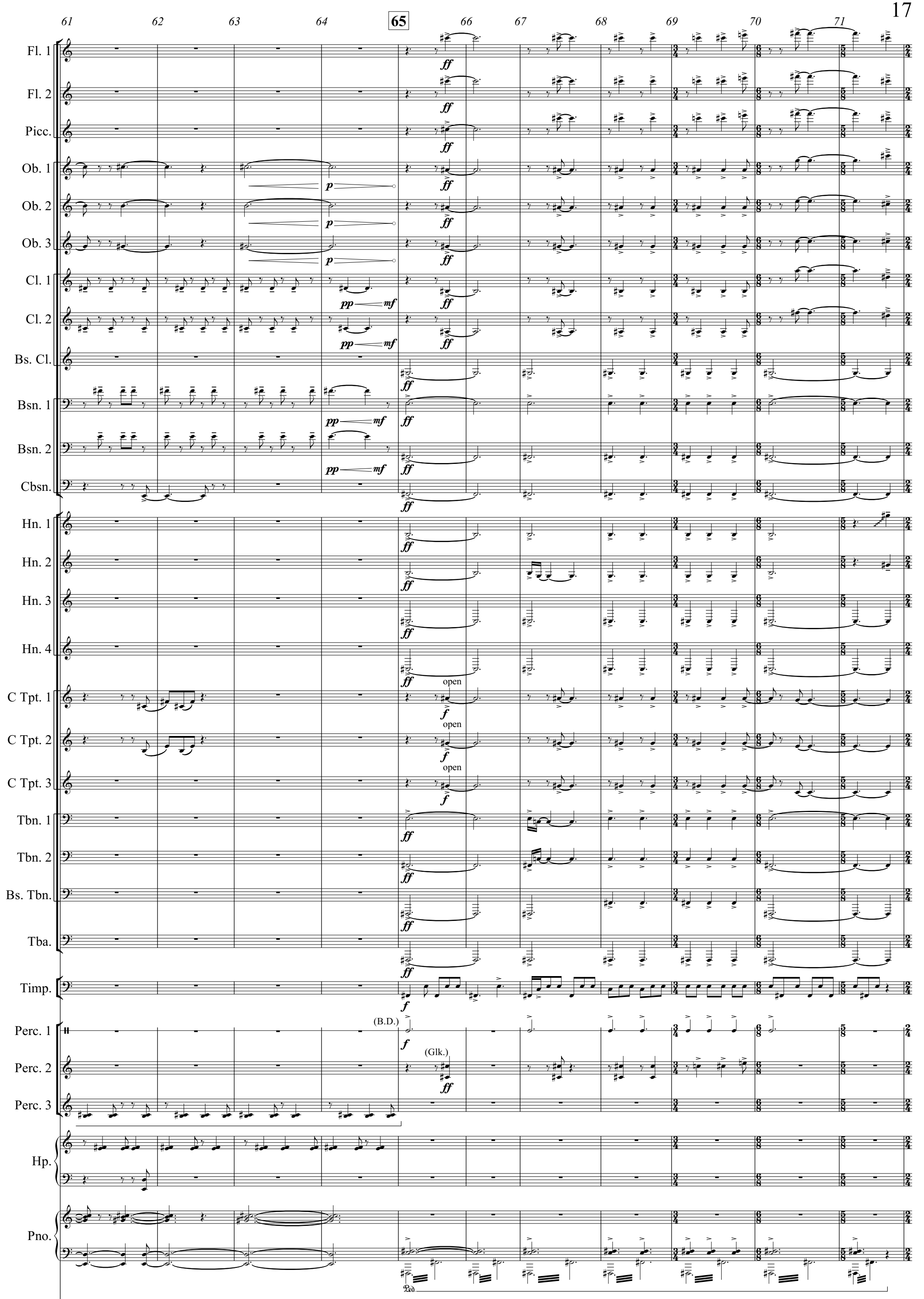

S. Solo $\mathrm{S}_{3}$ Vln. 2 Vln. 

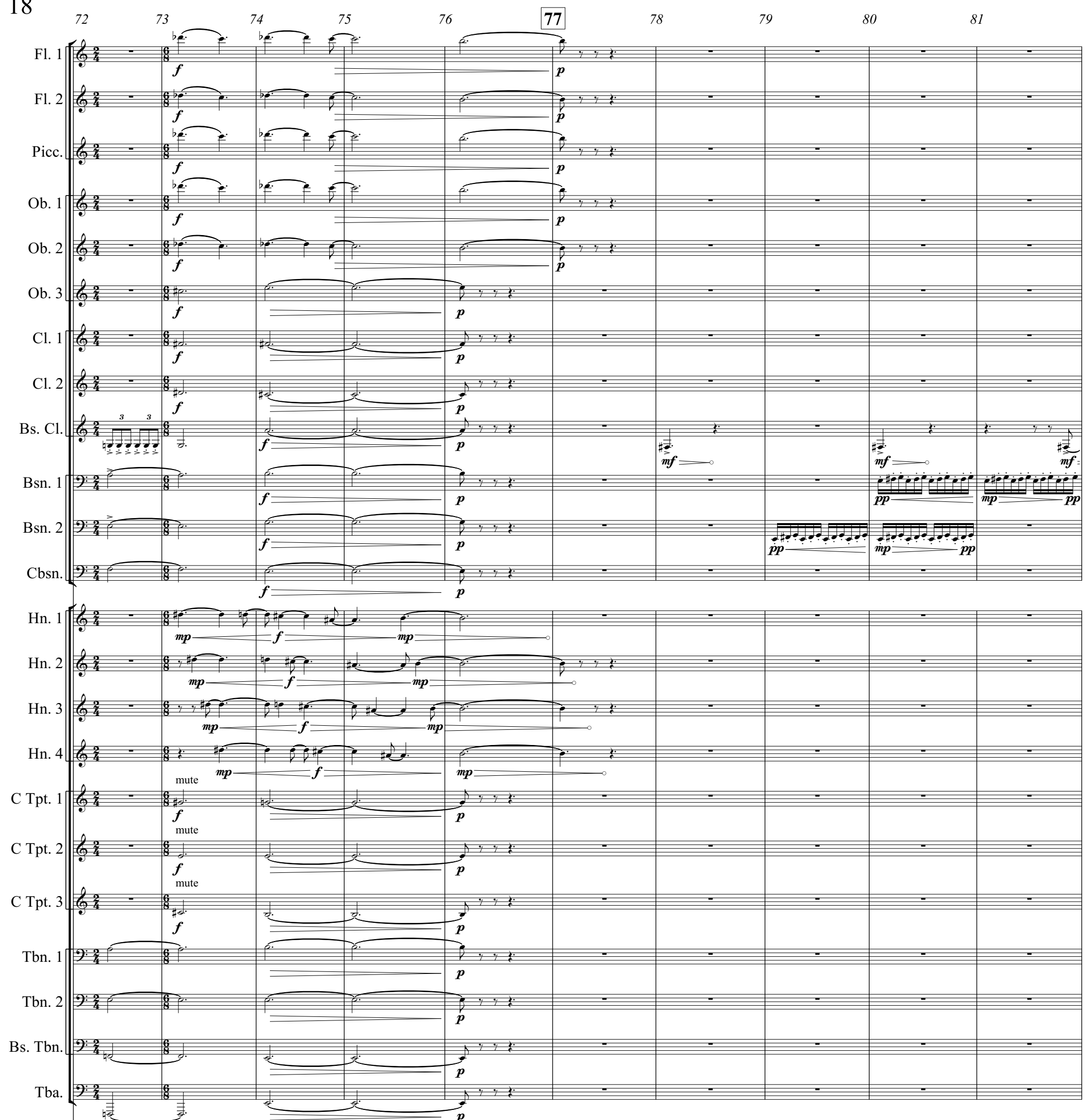
Timp. Perc. 2 Perc. 3 . 1 (G)

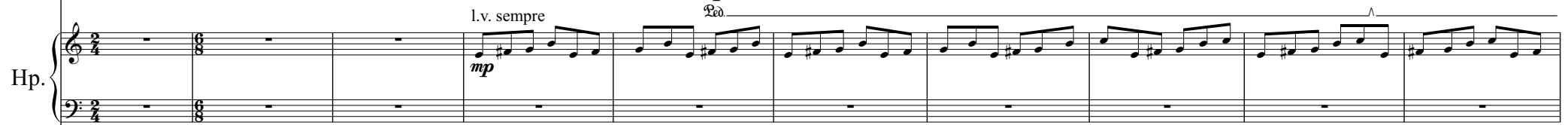

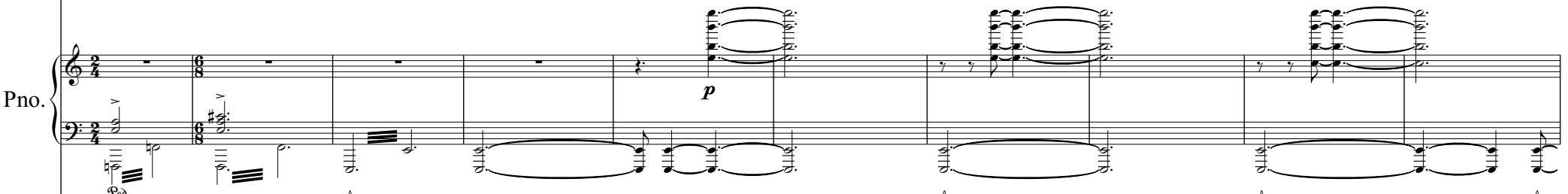

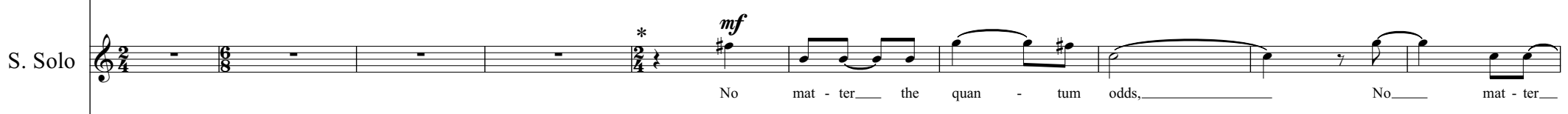

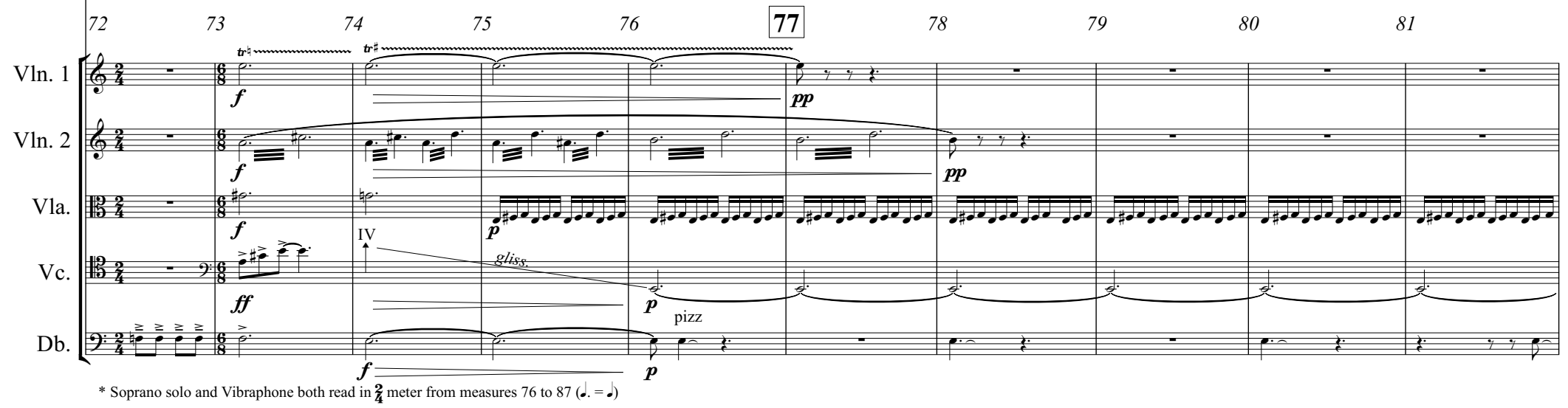




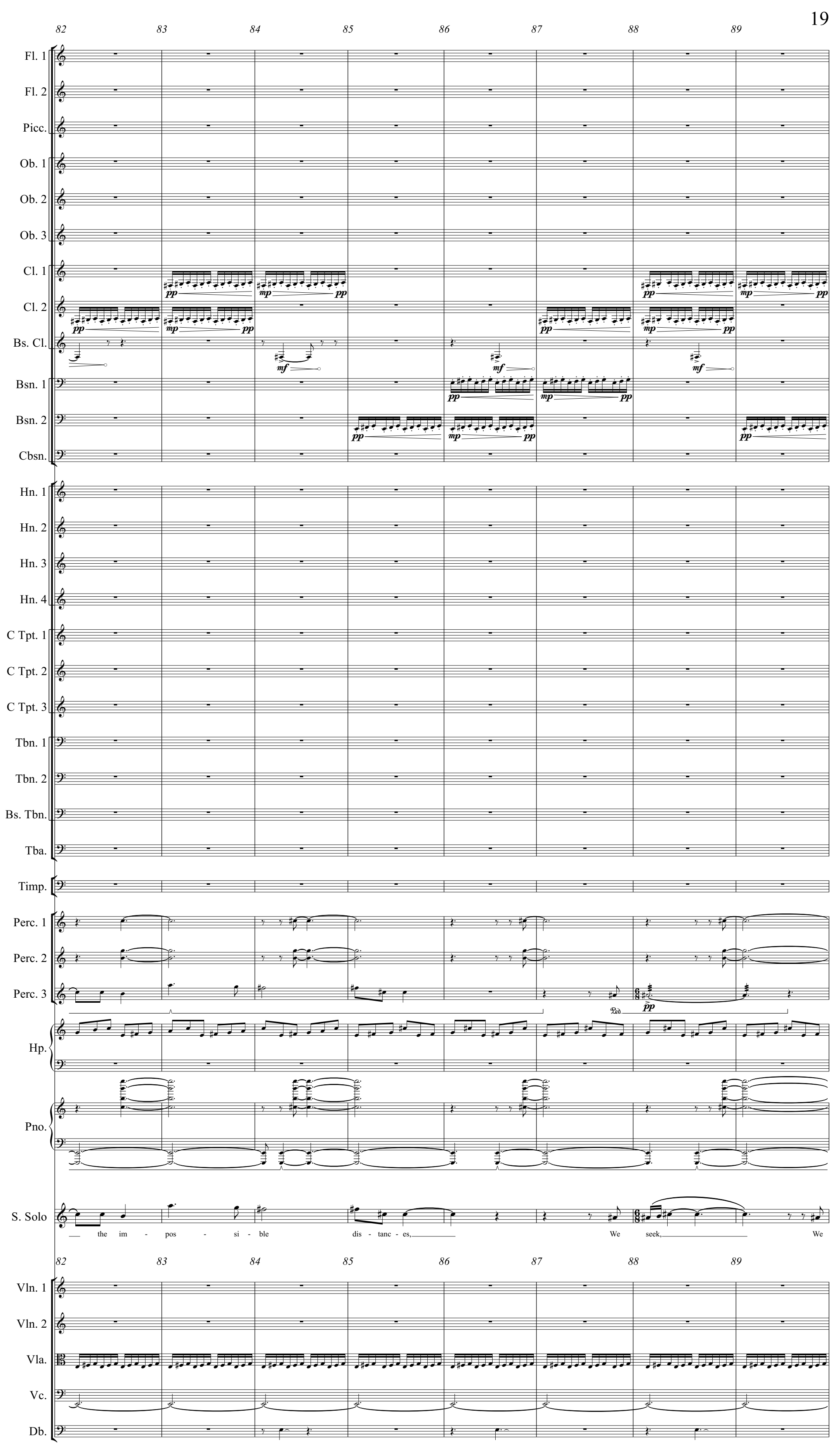




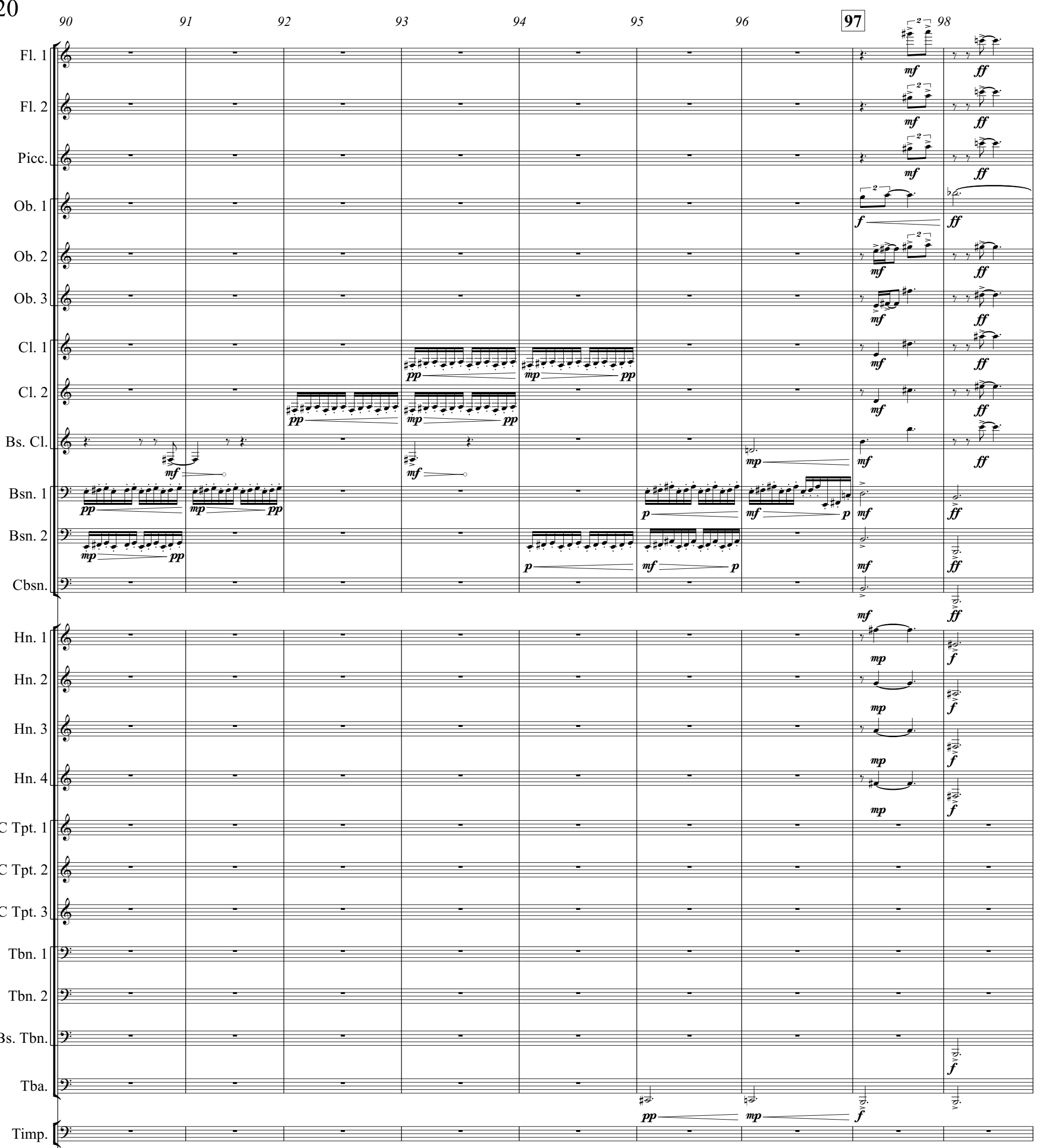

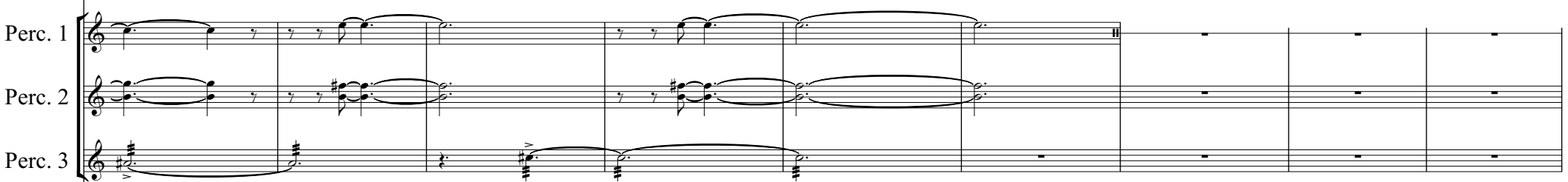

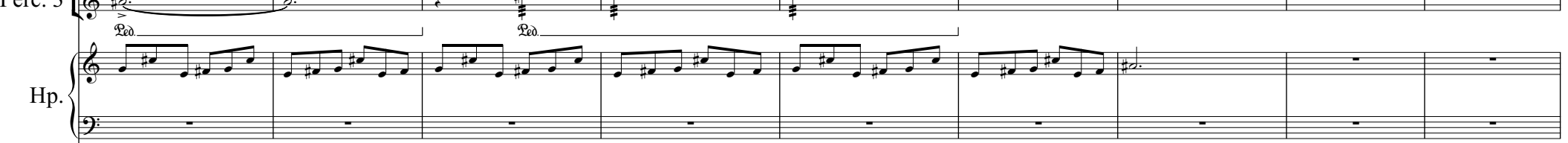
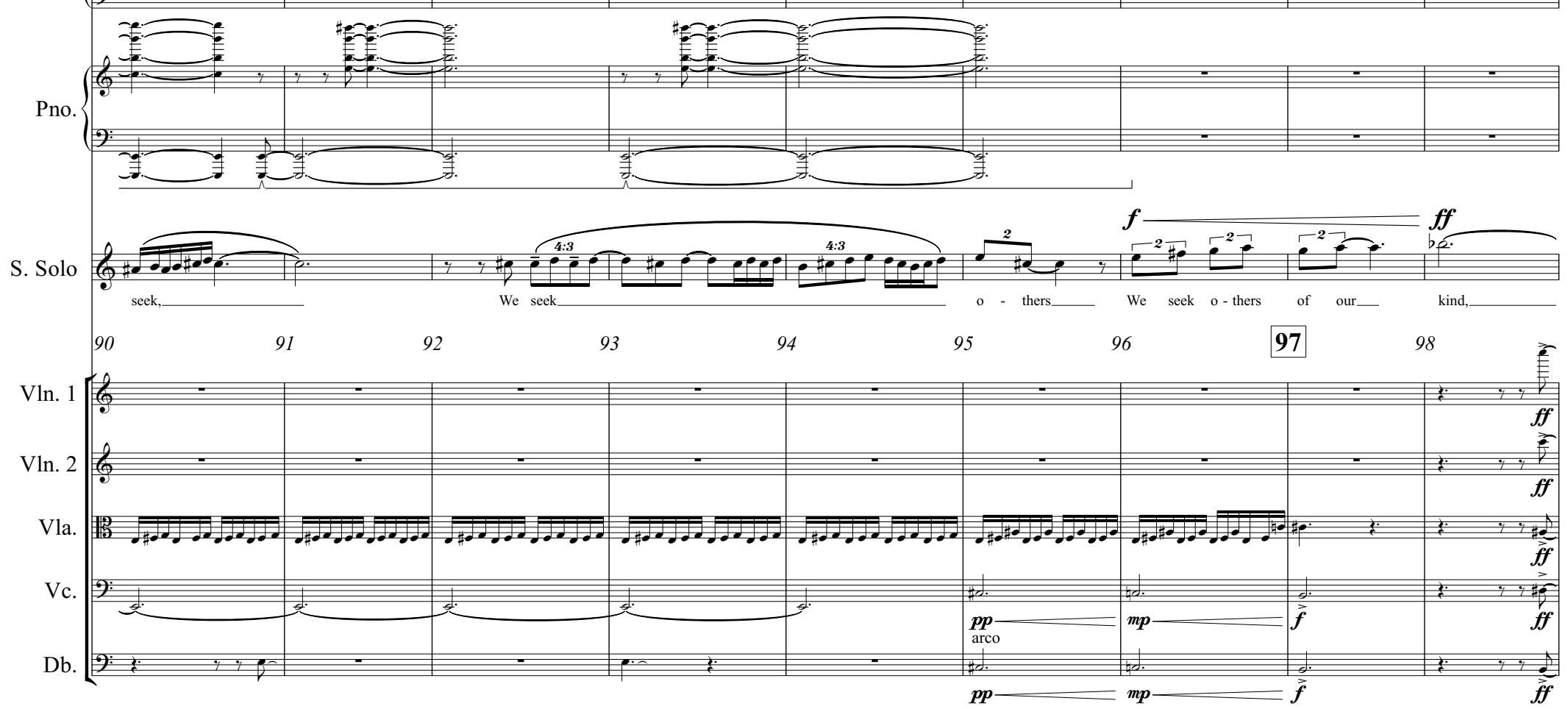


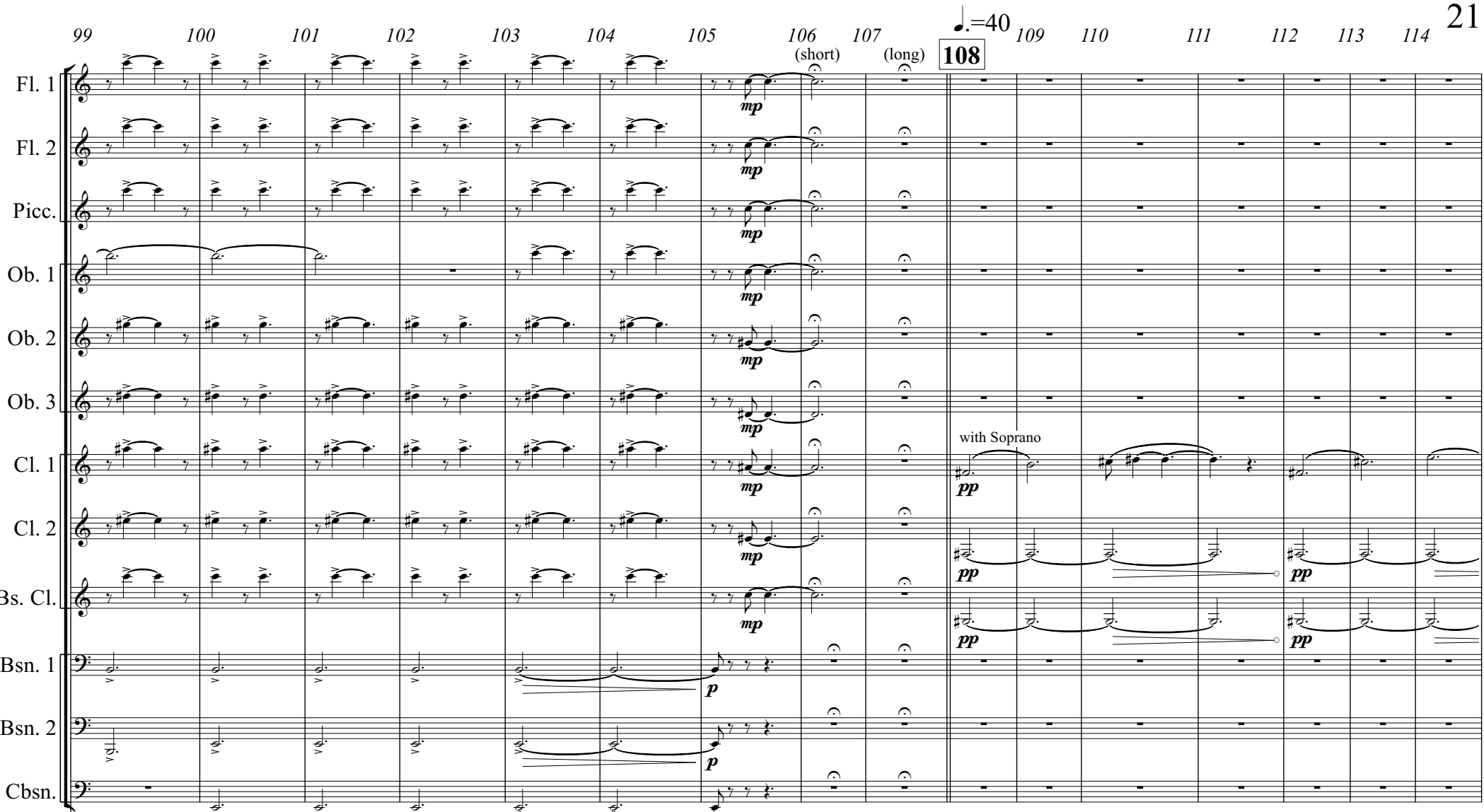
Cbsn. $\mathrm{H}$

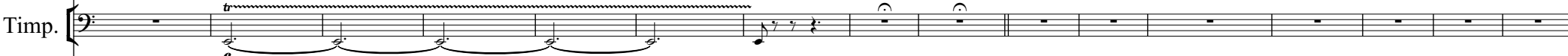
$\boldsymbol{f = ( 2 0 )}$

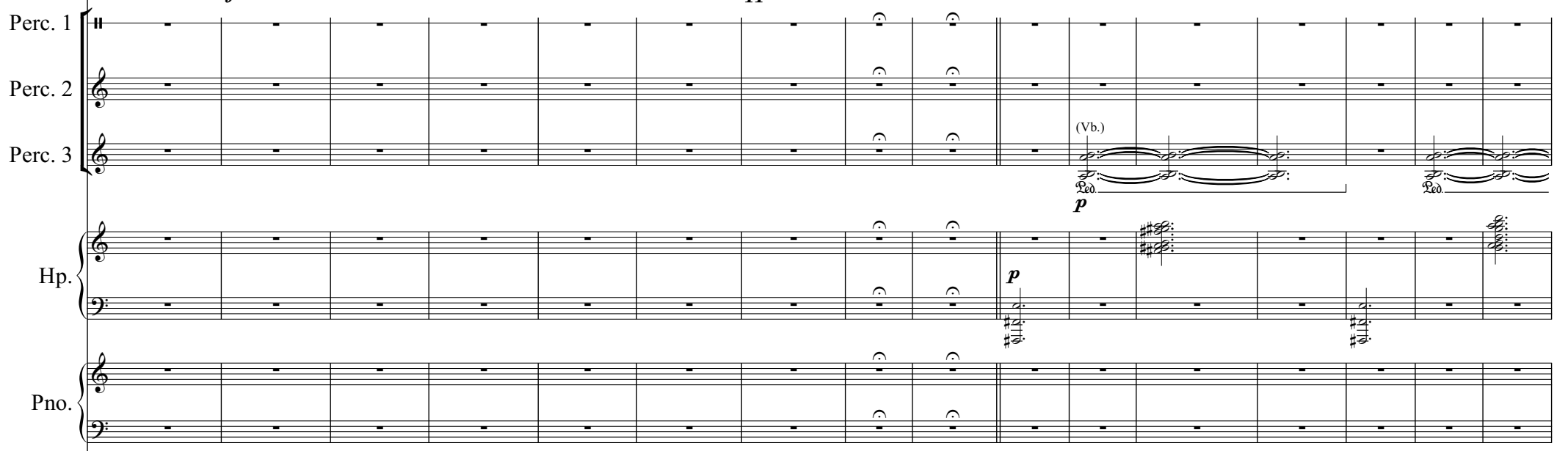

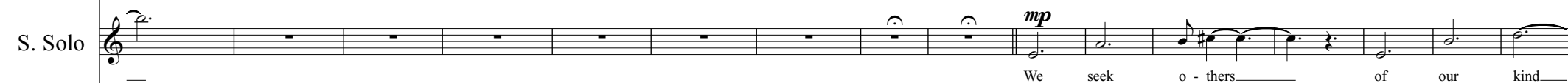

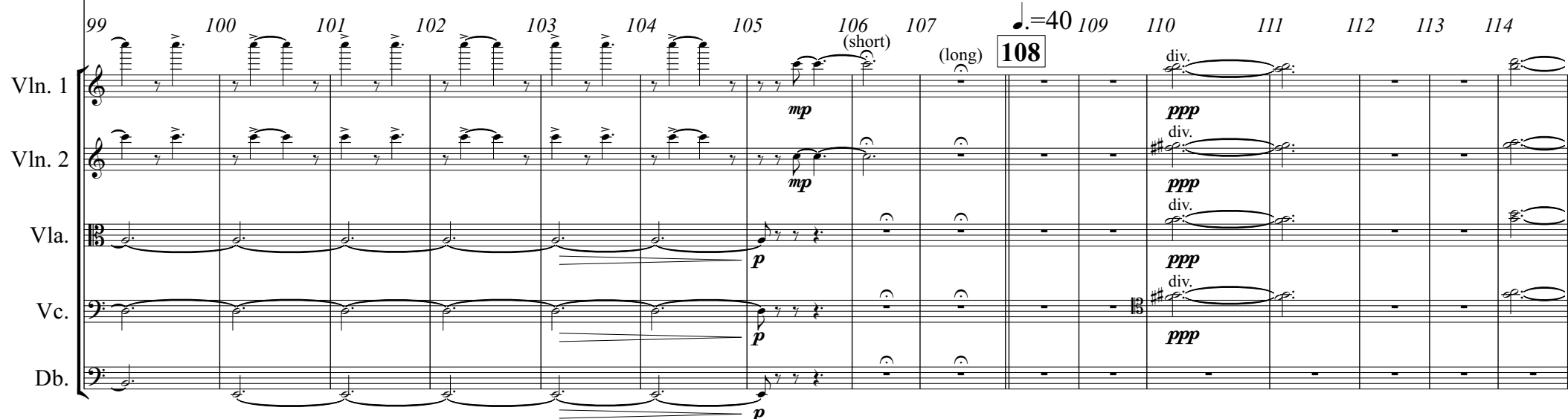



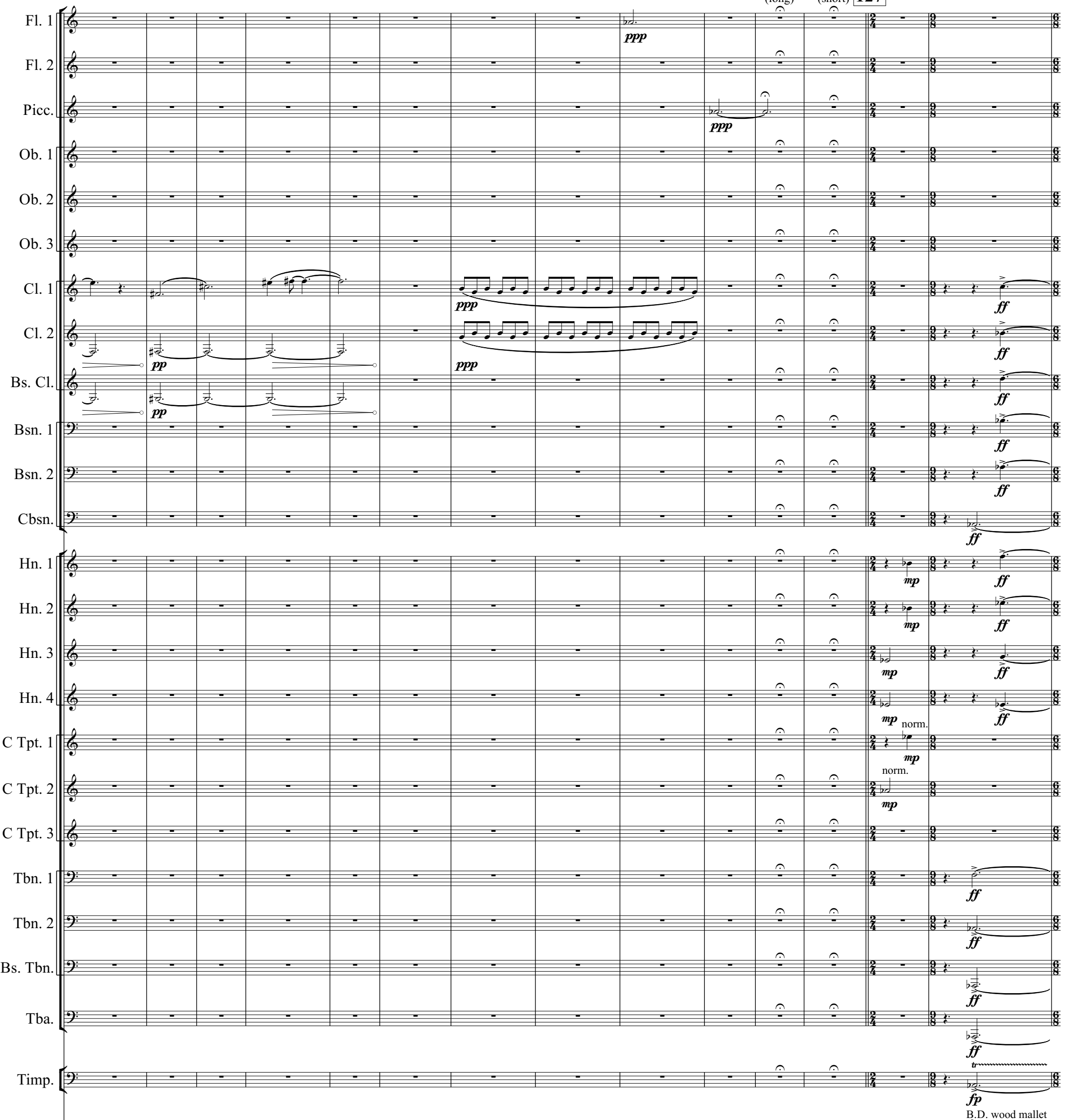

Perc. 1 |

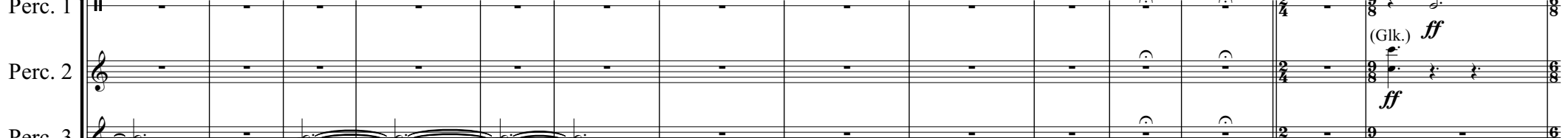

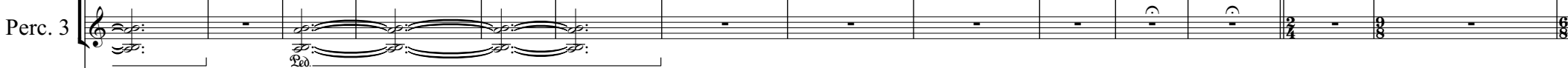

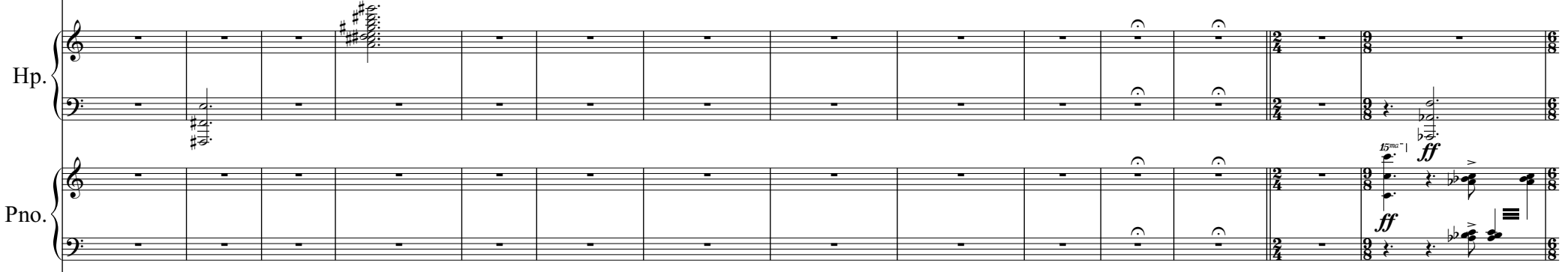

S. Solo

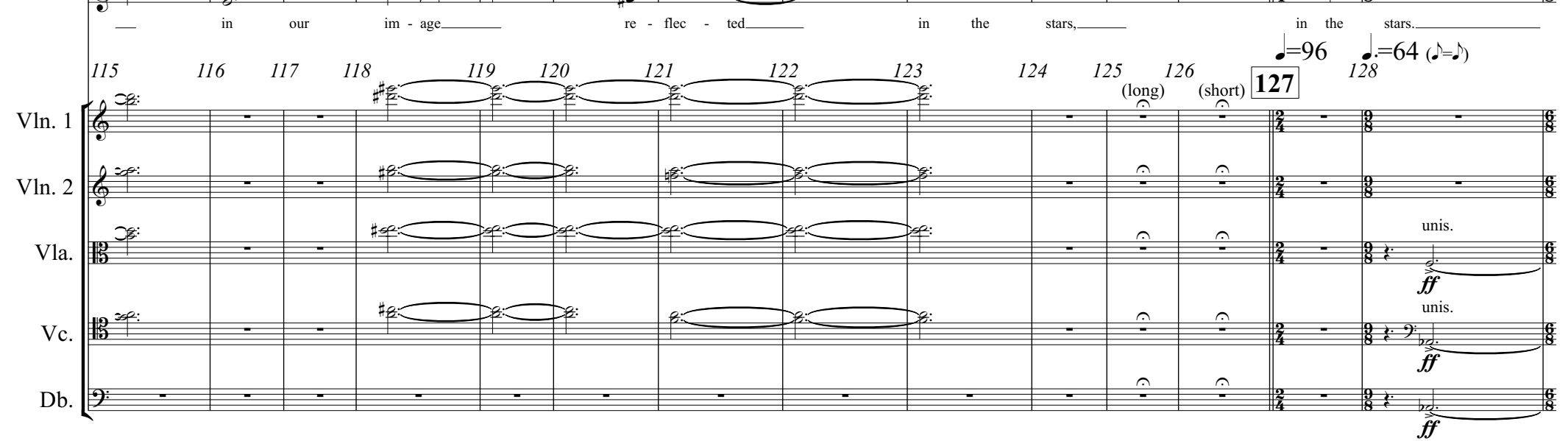




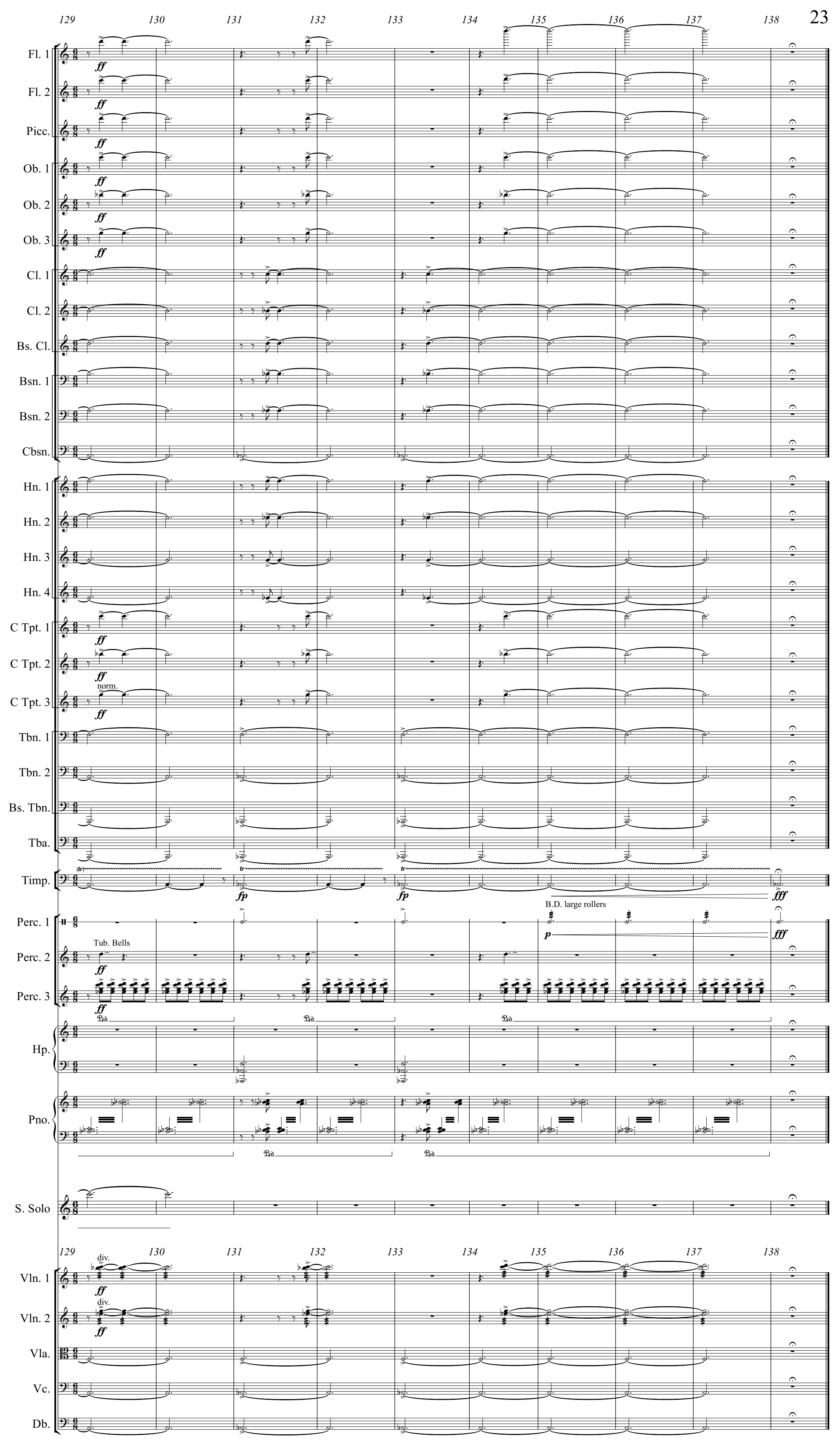




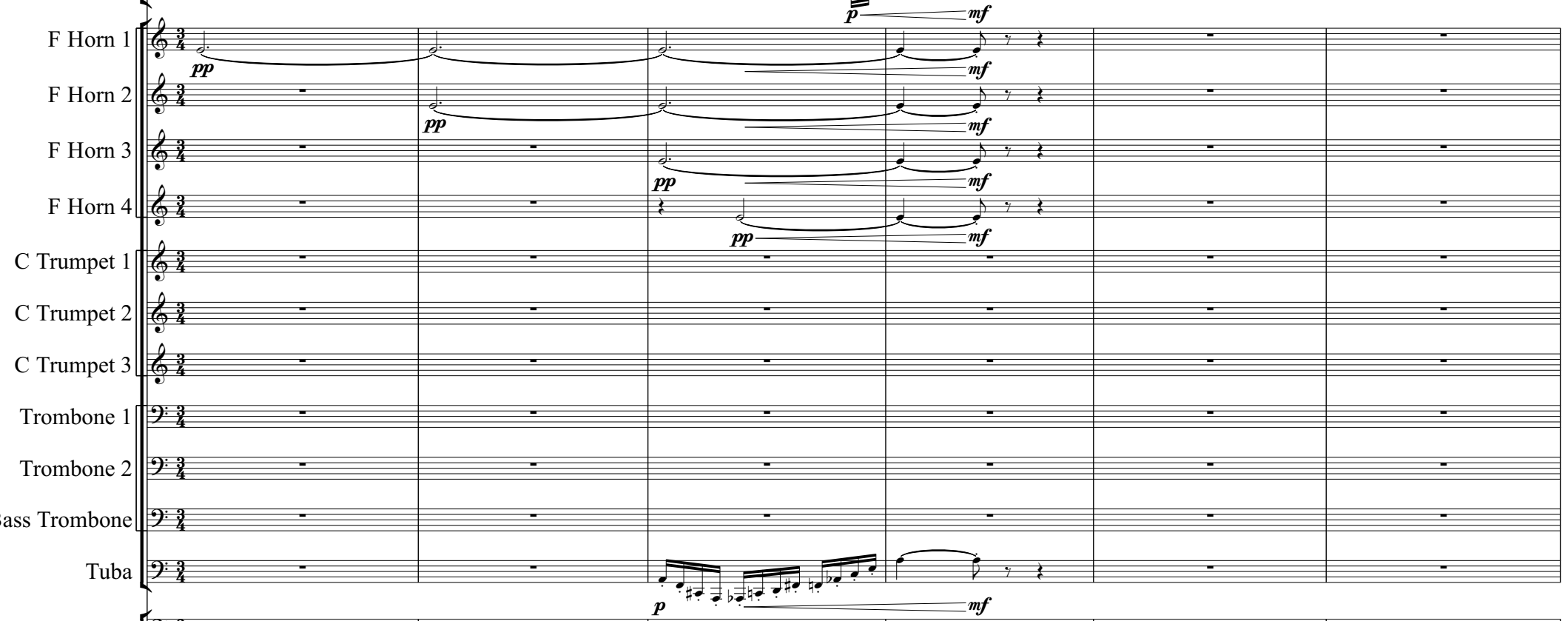

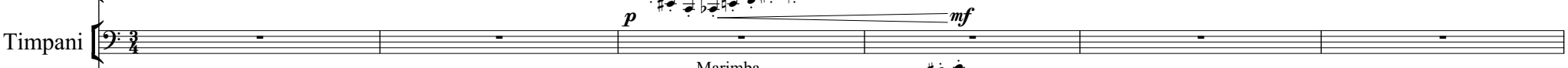

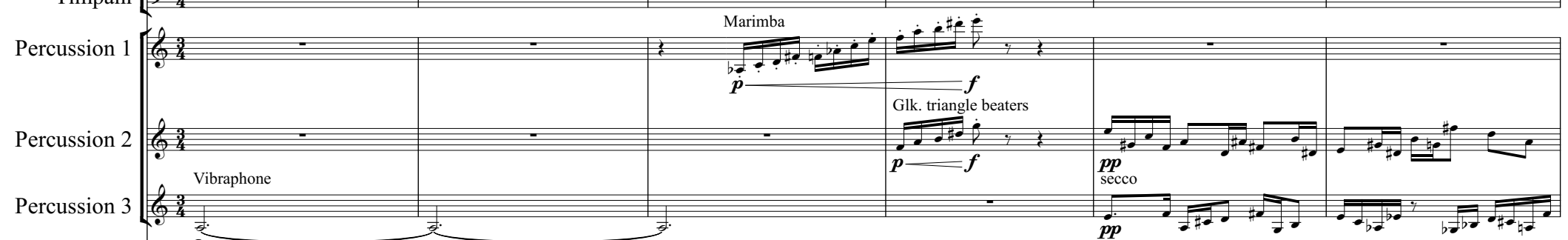

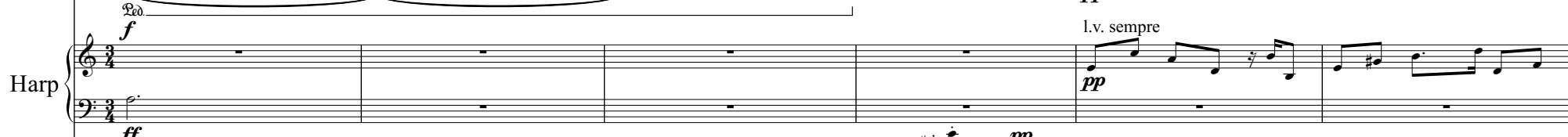

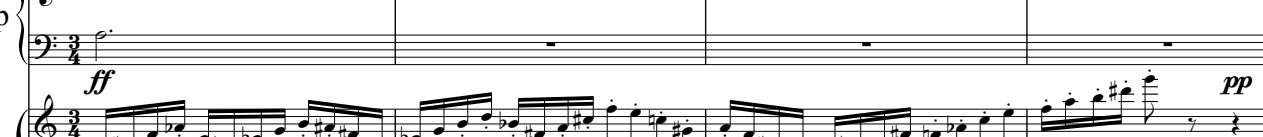

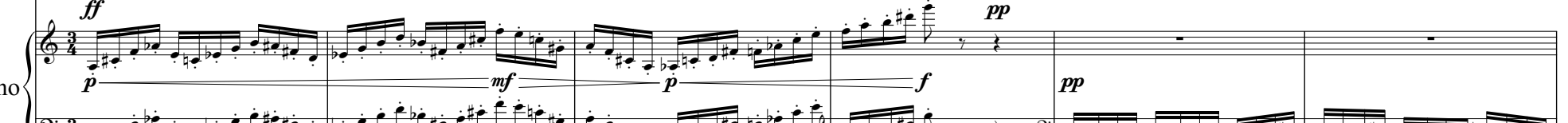
$2 y_{0}$

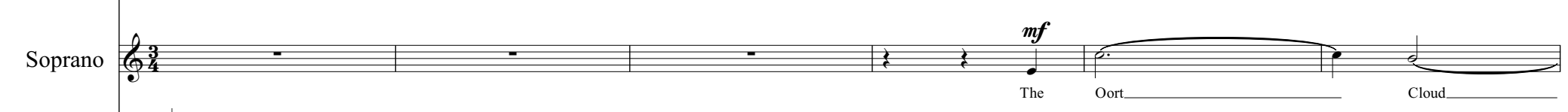

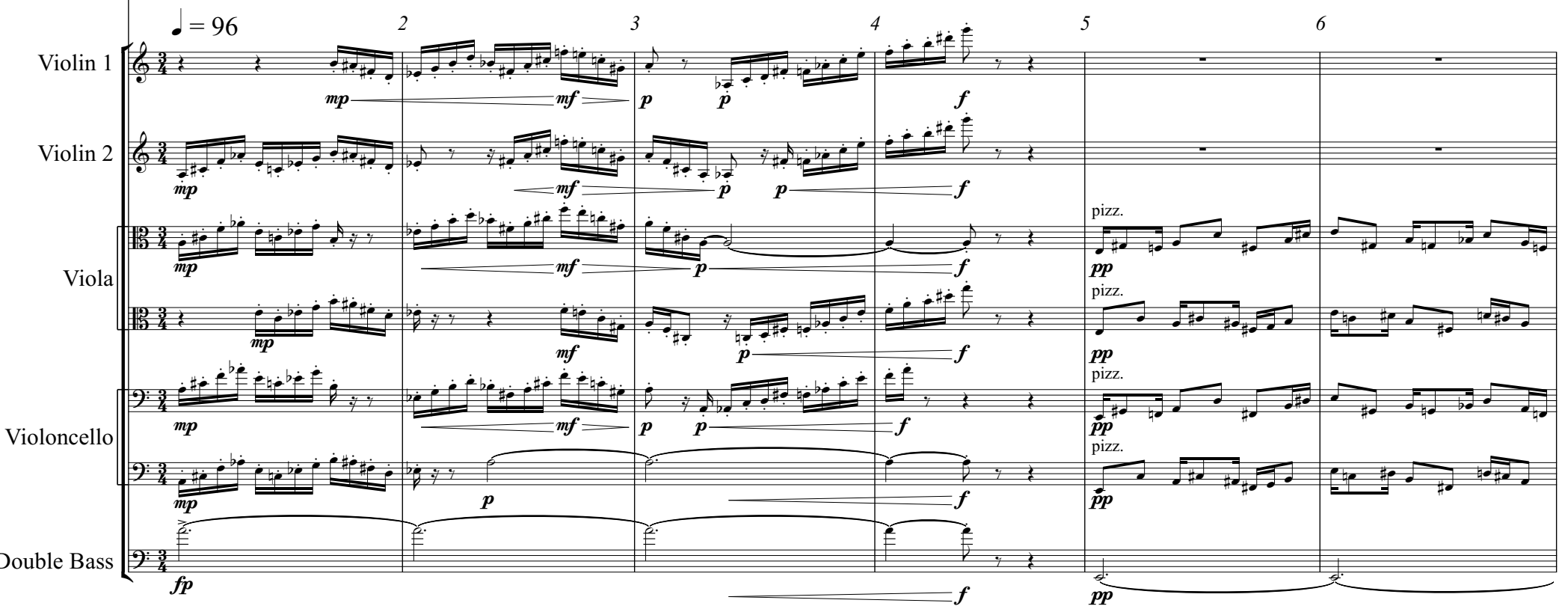



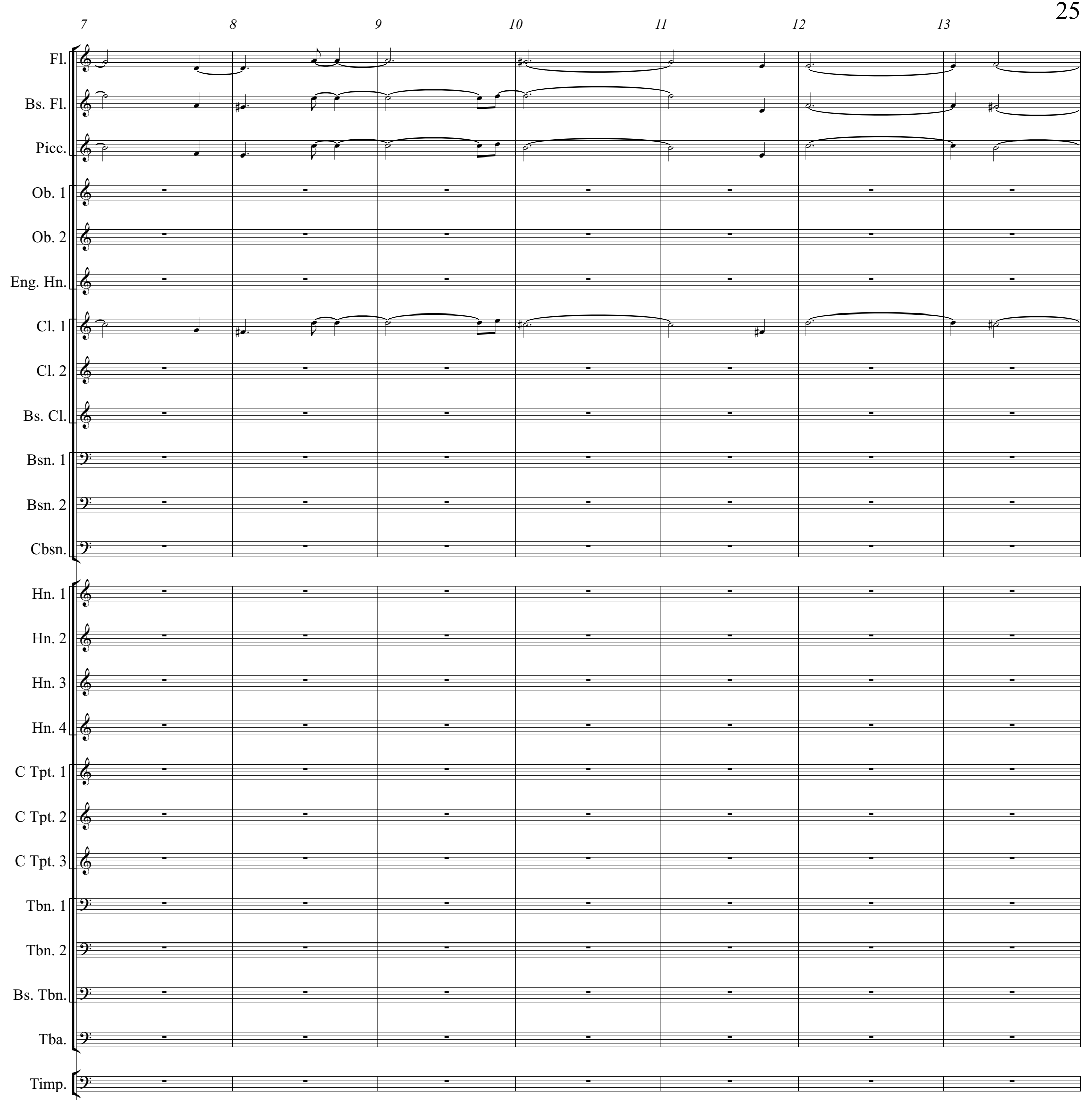

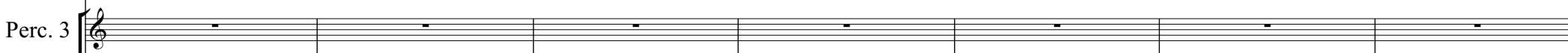
Perc 2 (6)

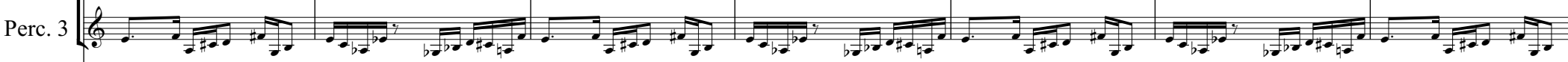

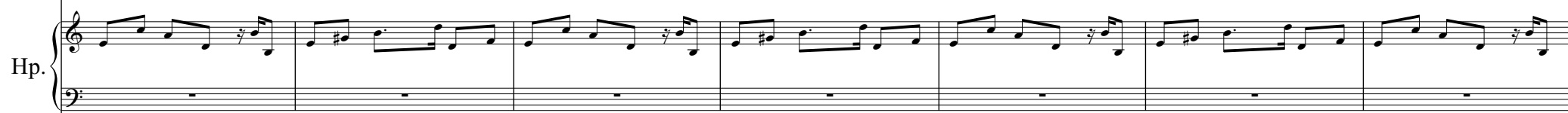

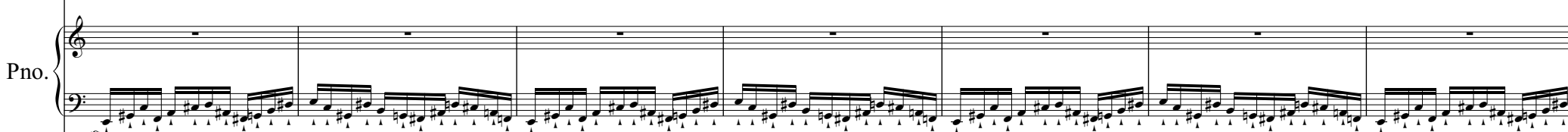
(is VIn 

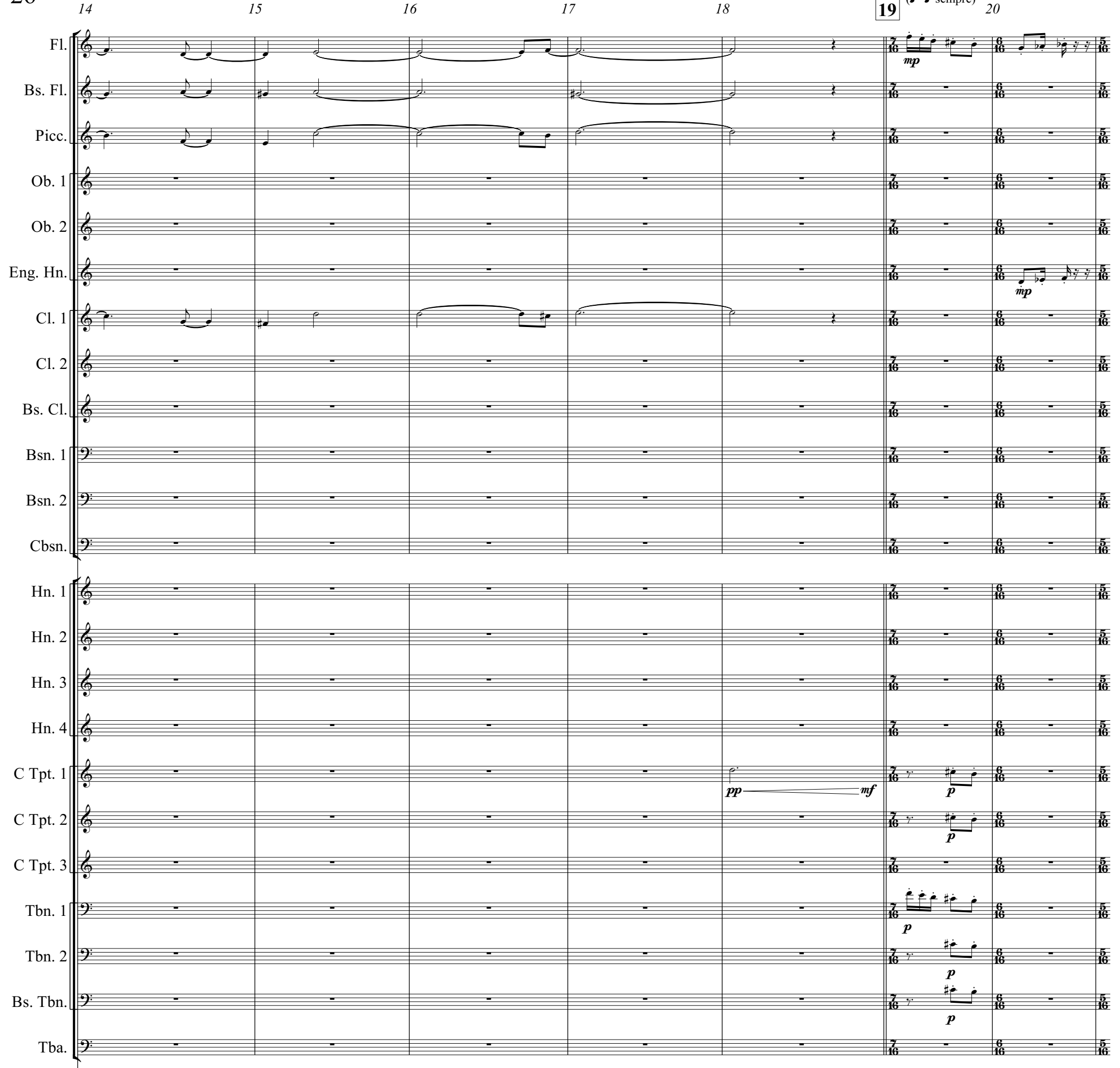

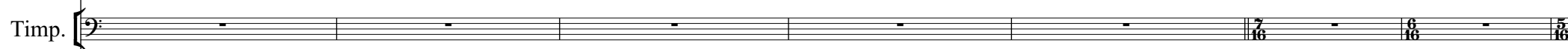

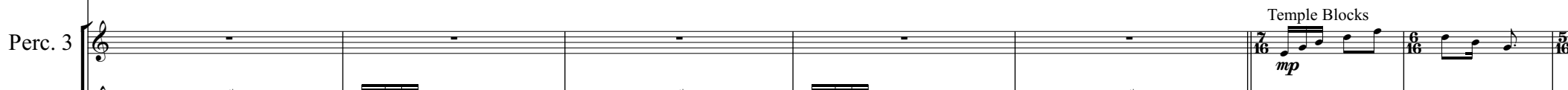

Perc. 2 (3)

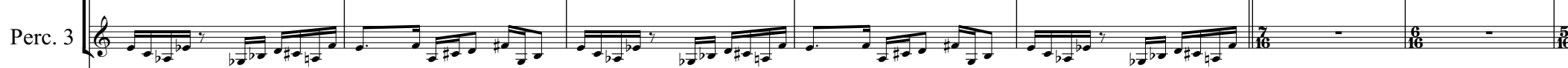

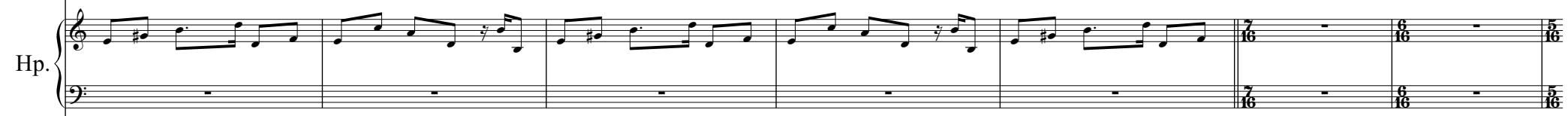
Pno. $\left.\right|_{3} ^{3}$

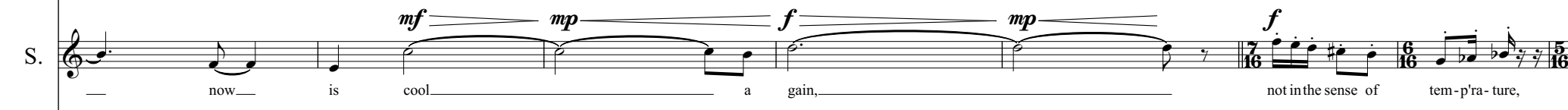
Vln. 2 

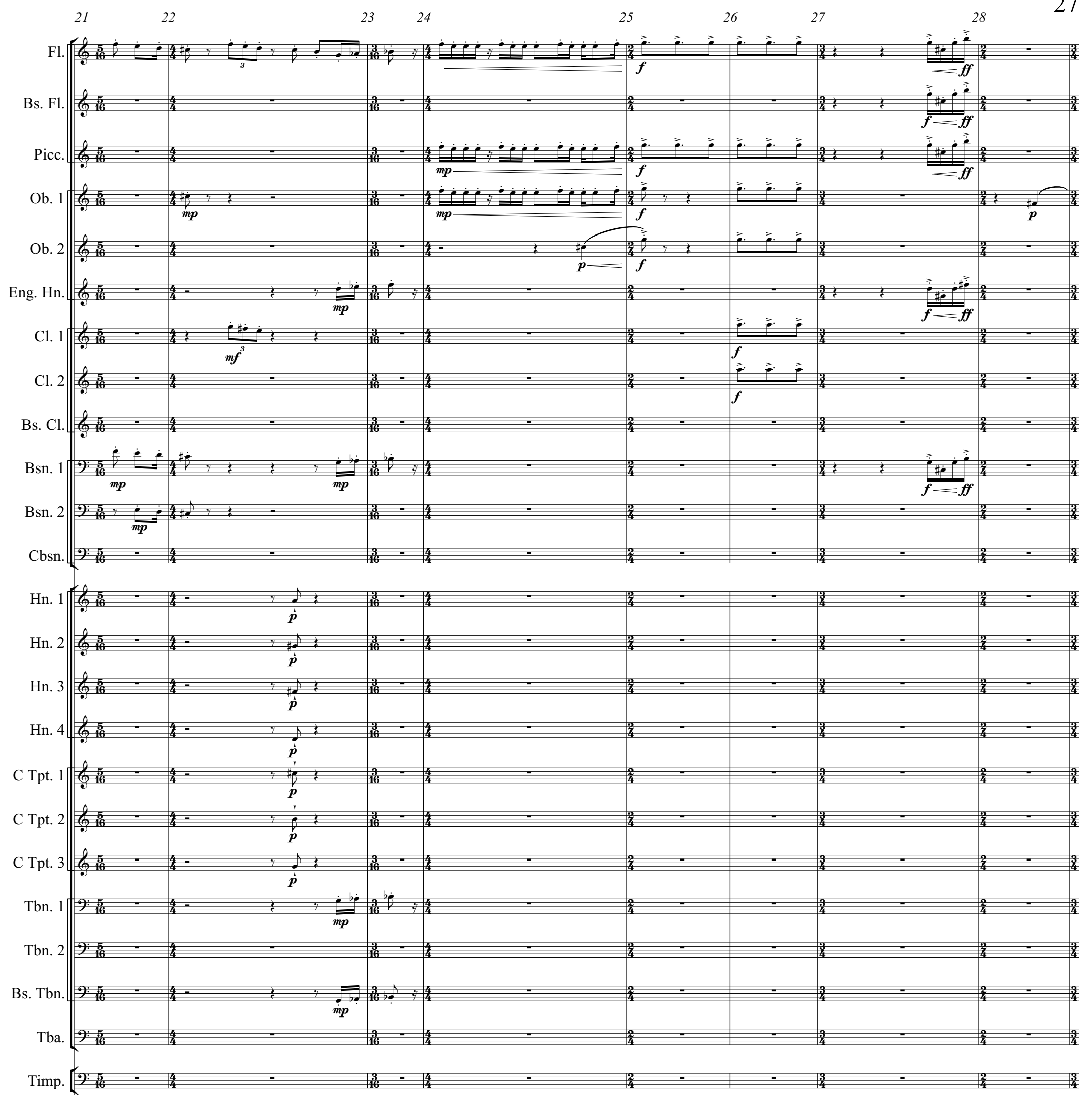

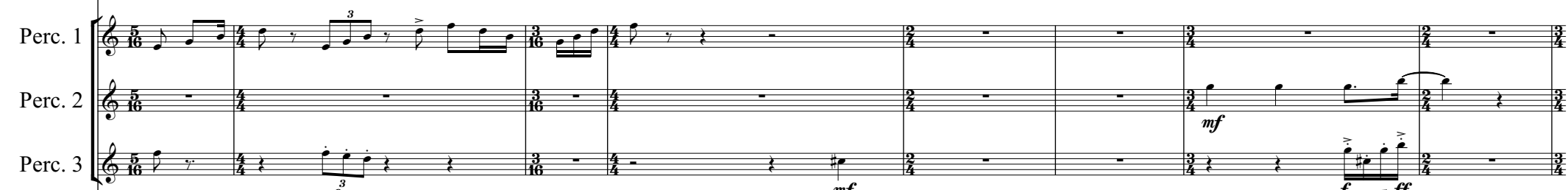

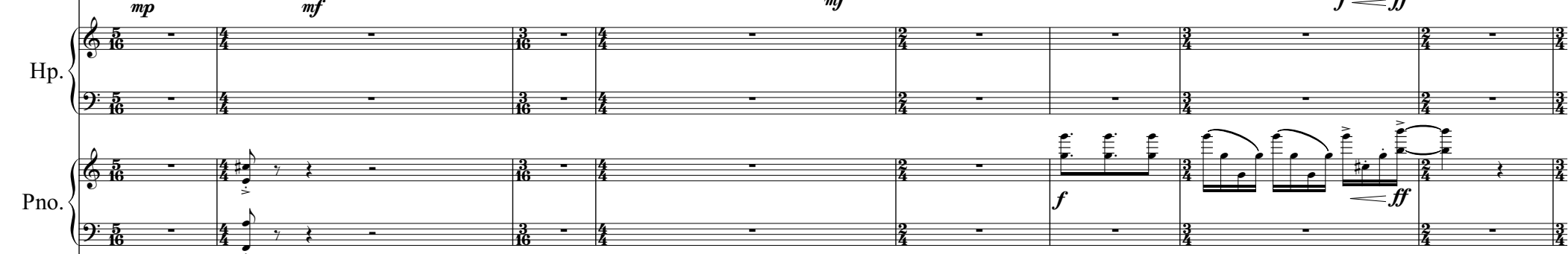

S.

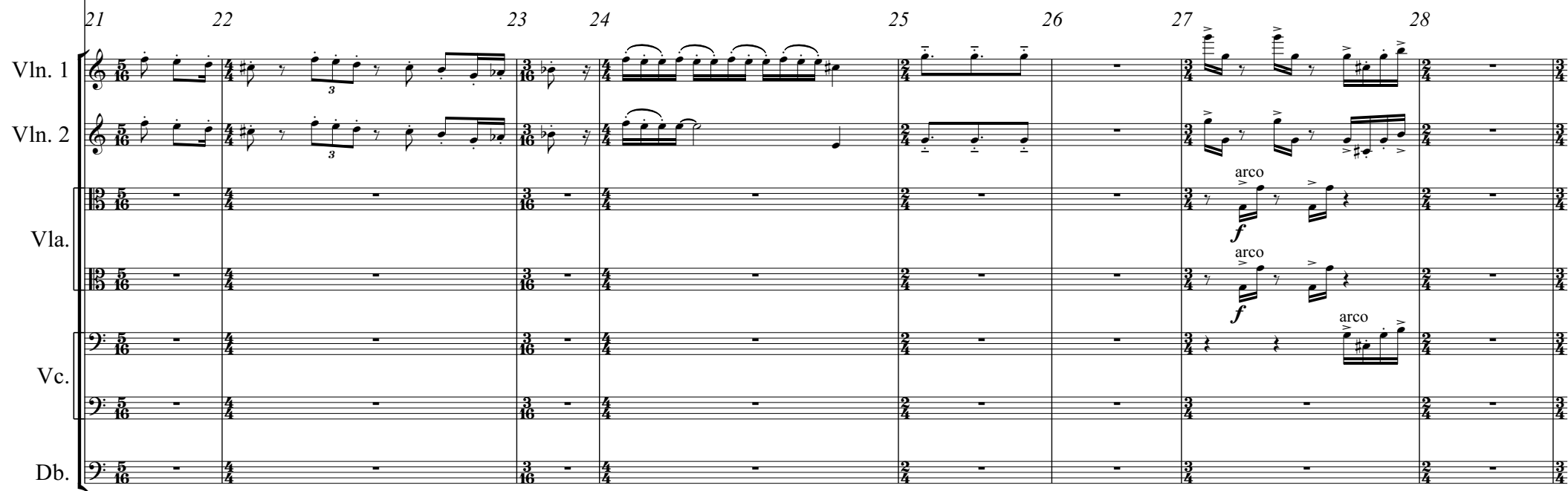



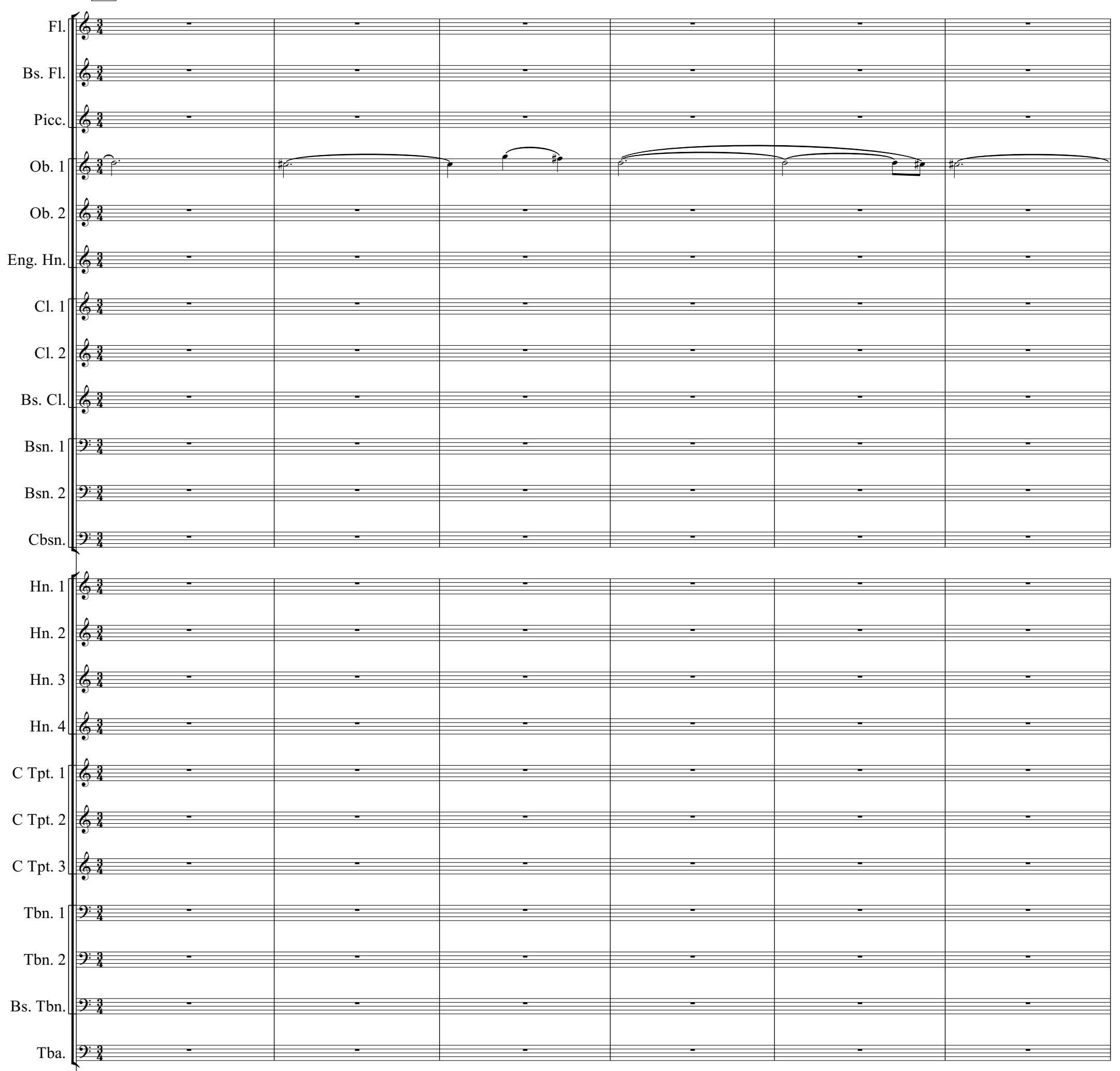

Timp.

Perc. 1

Perc. 2 (3)

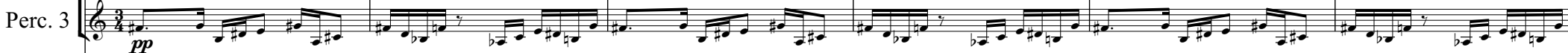

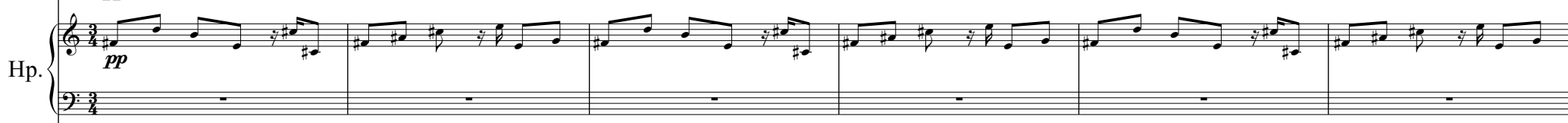

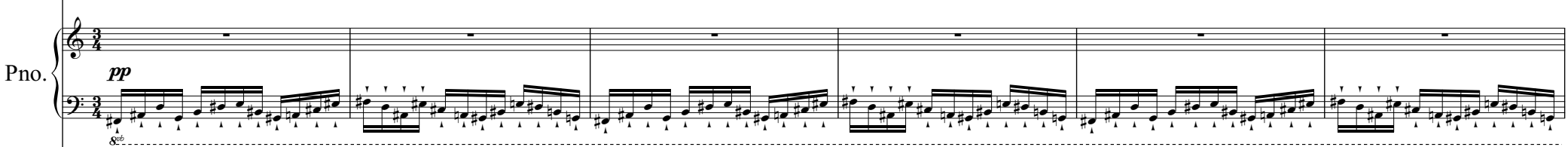

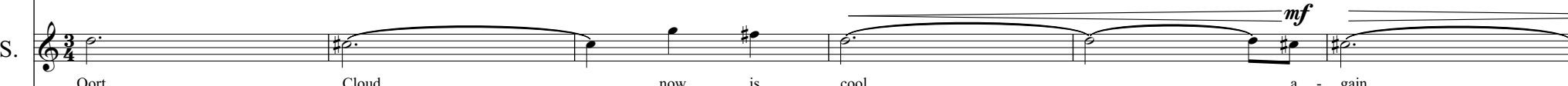

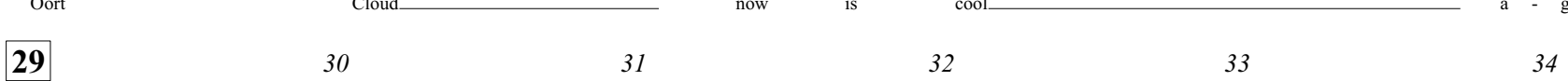

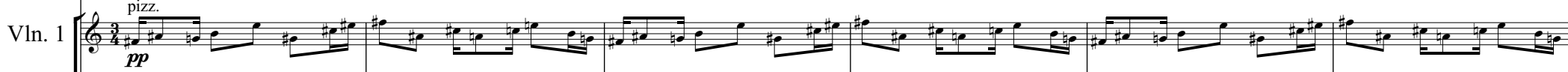

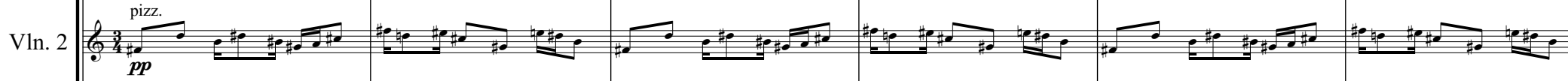

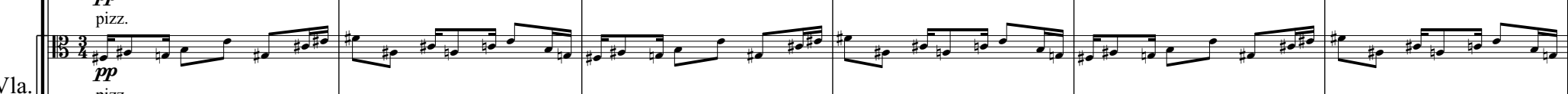
Vla. $\frac{\text { pizz. }}{p p}$ Vc.

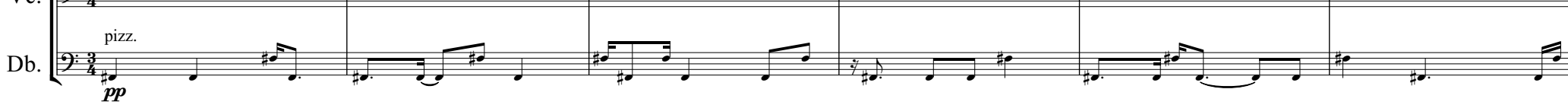




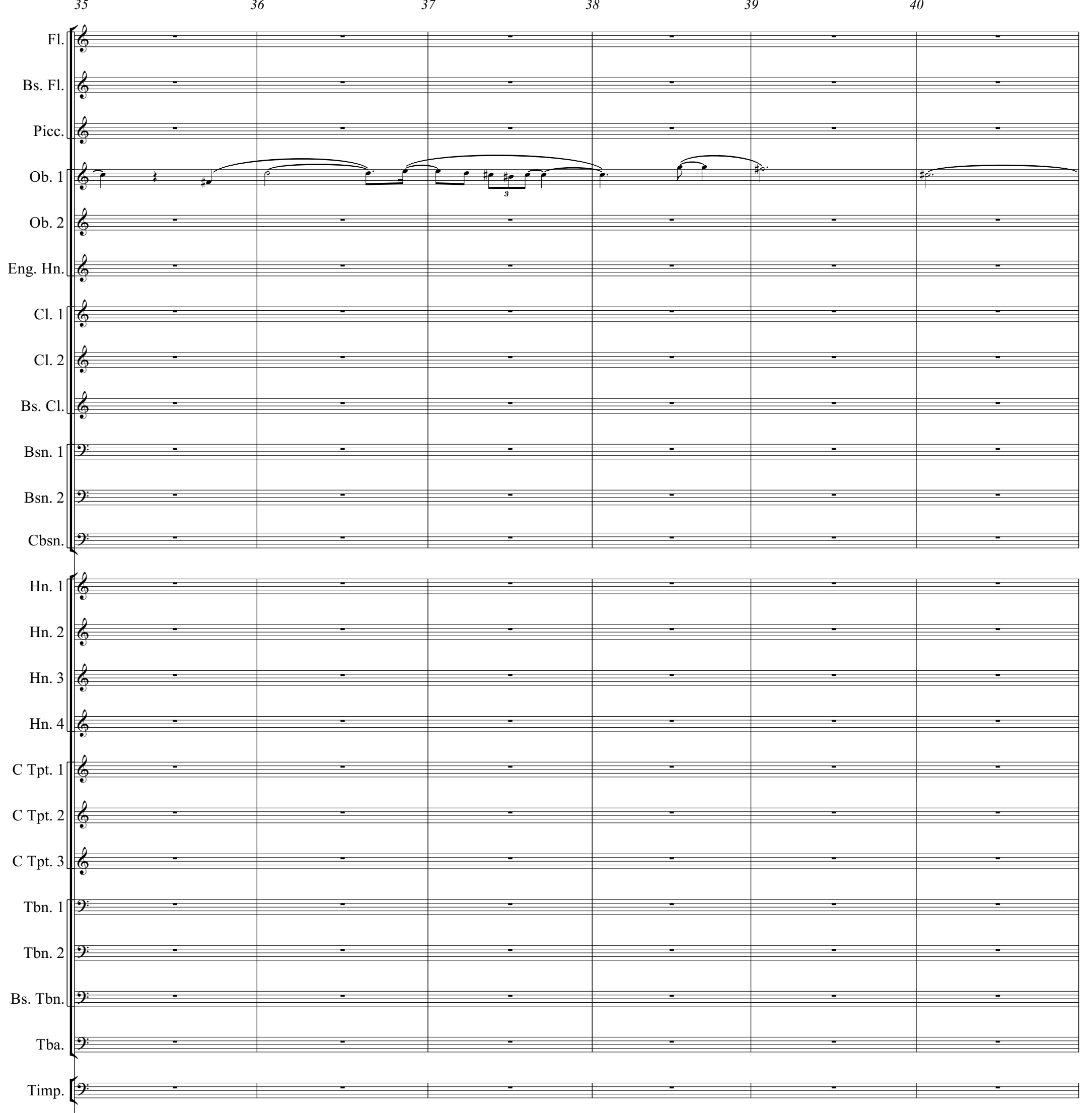

Perc. 2 Perc. 3

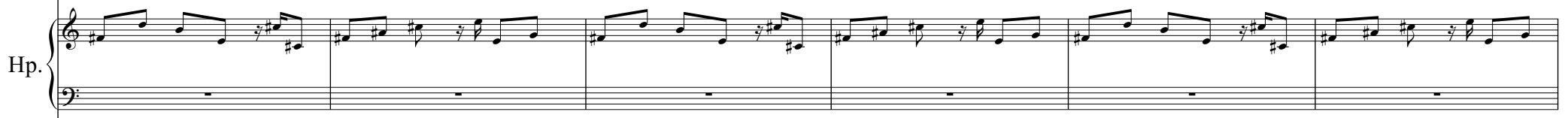

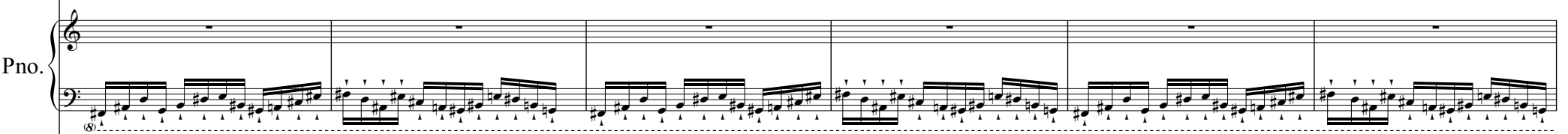
s. (a) $\begin{array}{lllllll}35 & 36 & 37 & 38 & 39 & 40\end{array}$

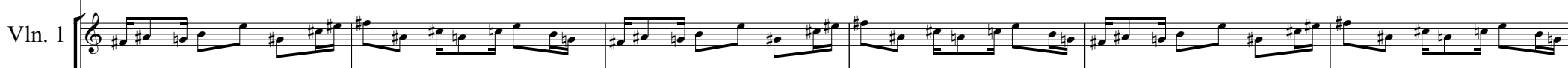

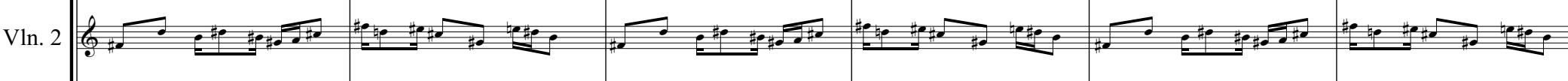

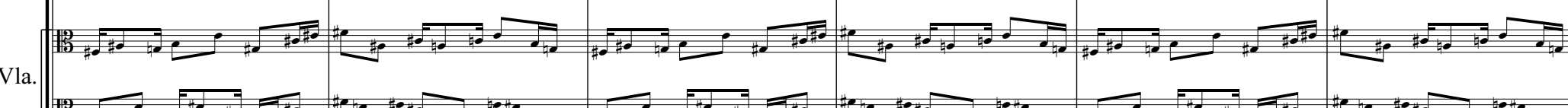

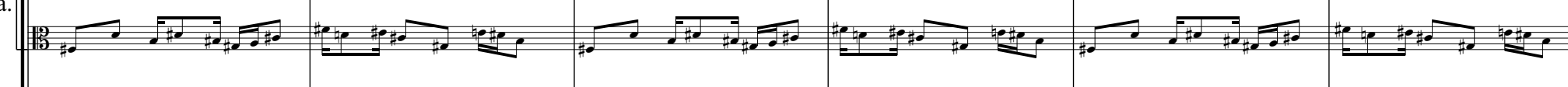
vc.

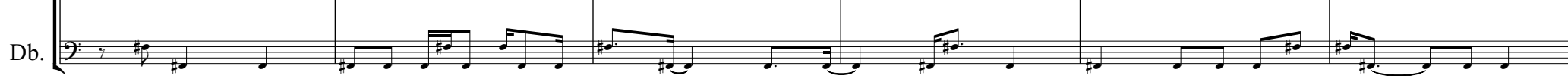



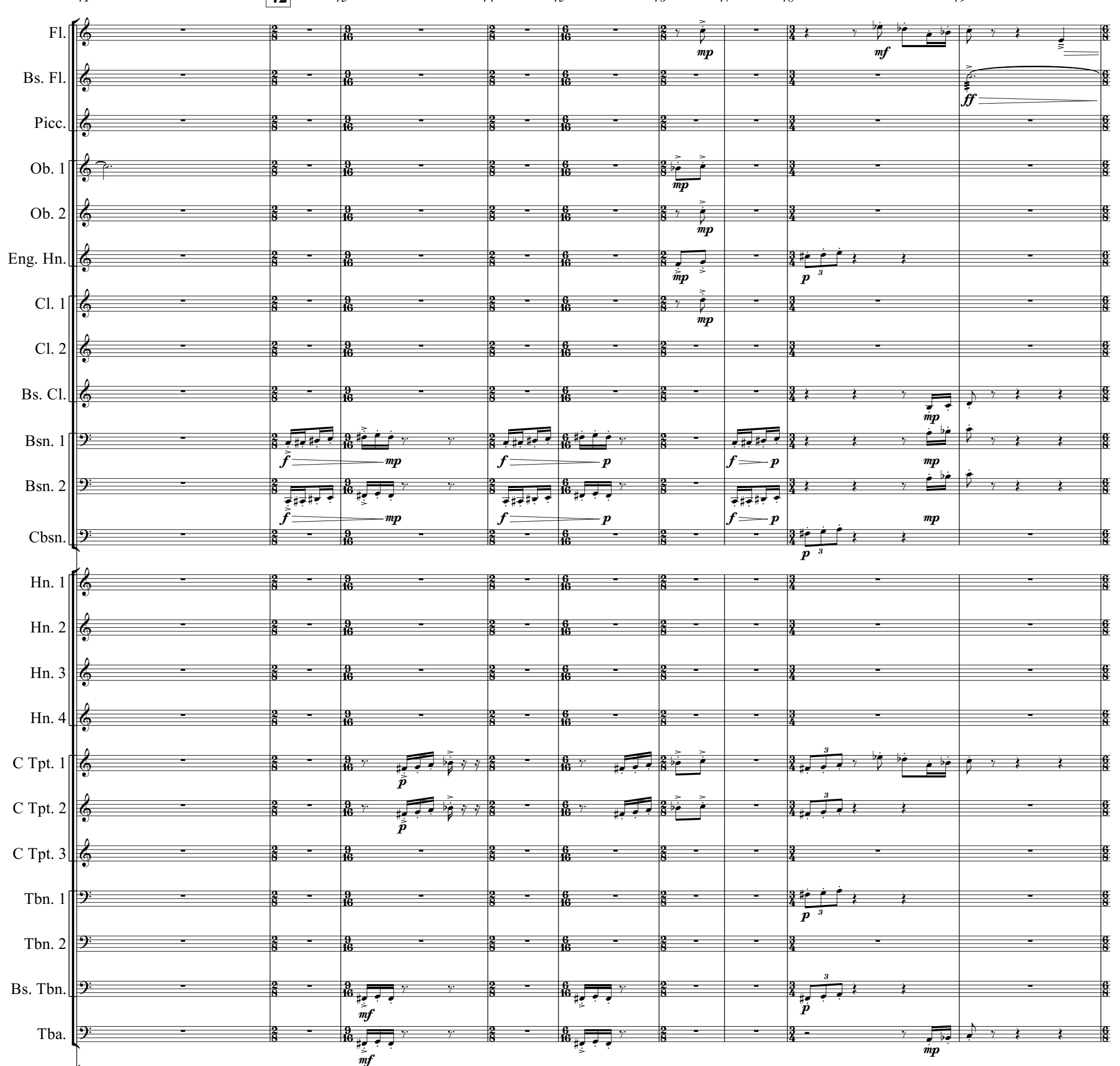

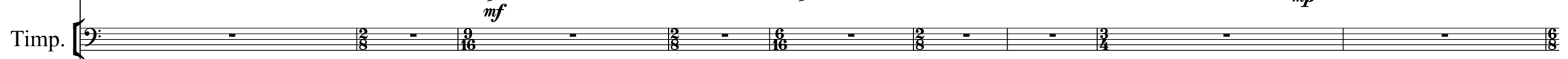

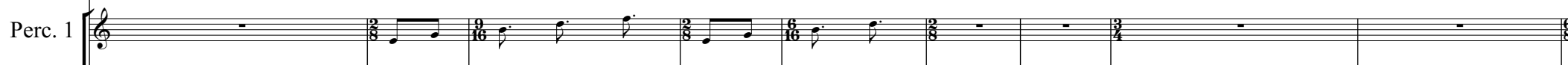

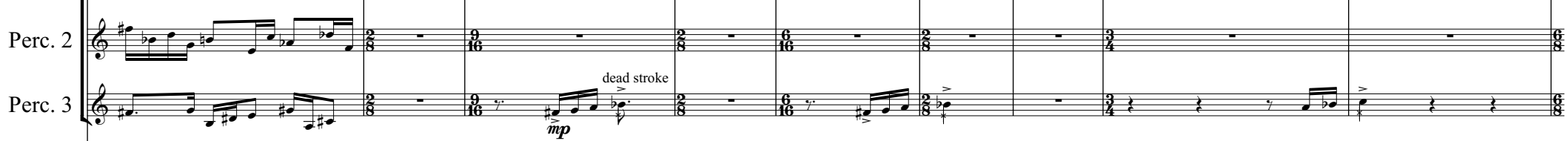

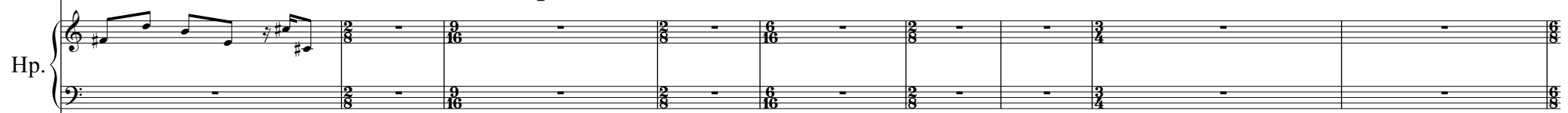

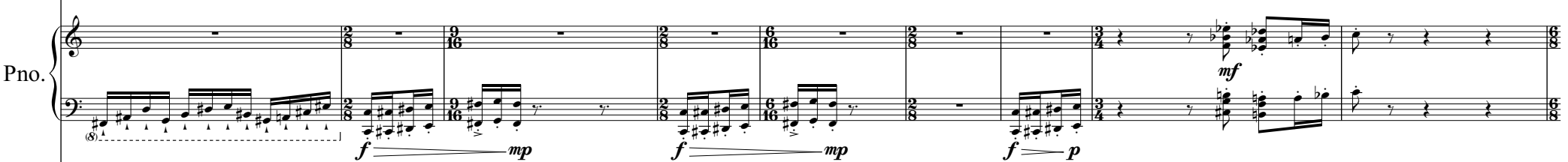
$m f$ \begin{tabular}{|l|llllllll}
41 & 42 & 43 & 44 & 45 & 46 & 47 & 48 & 49
\end{tabular}

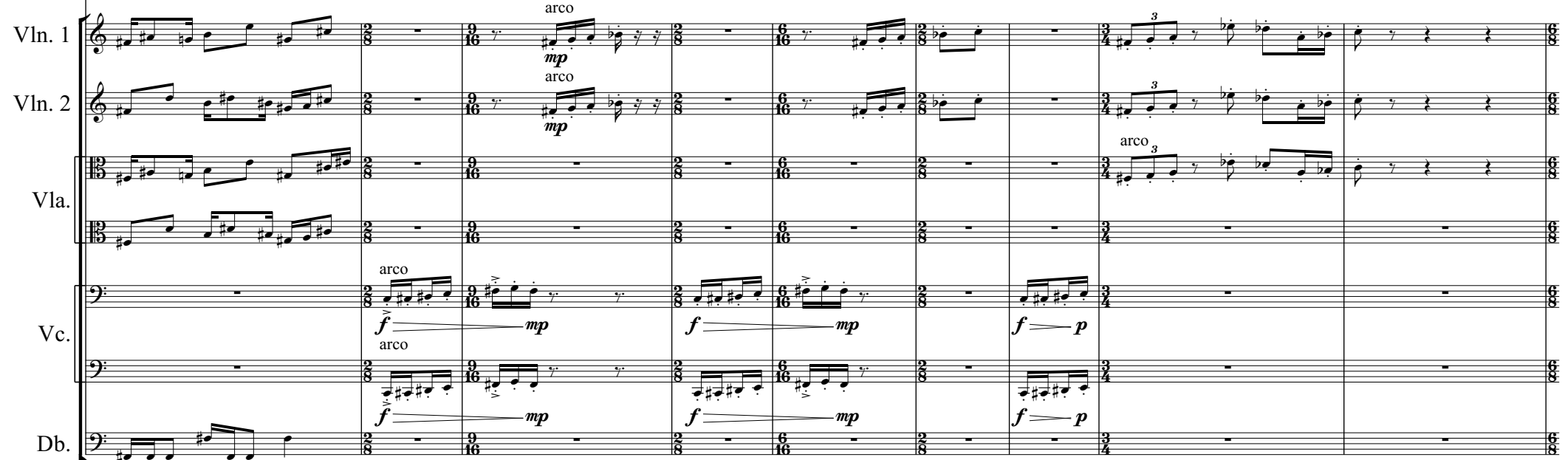



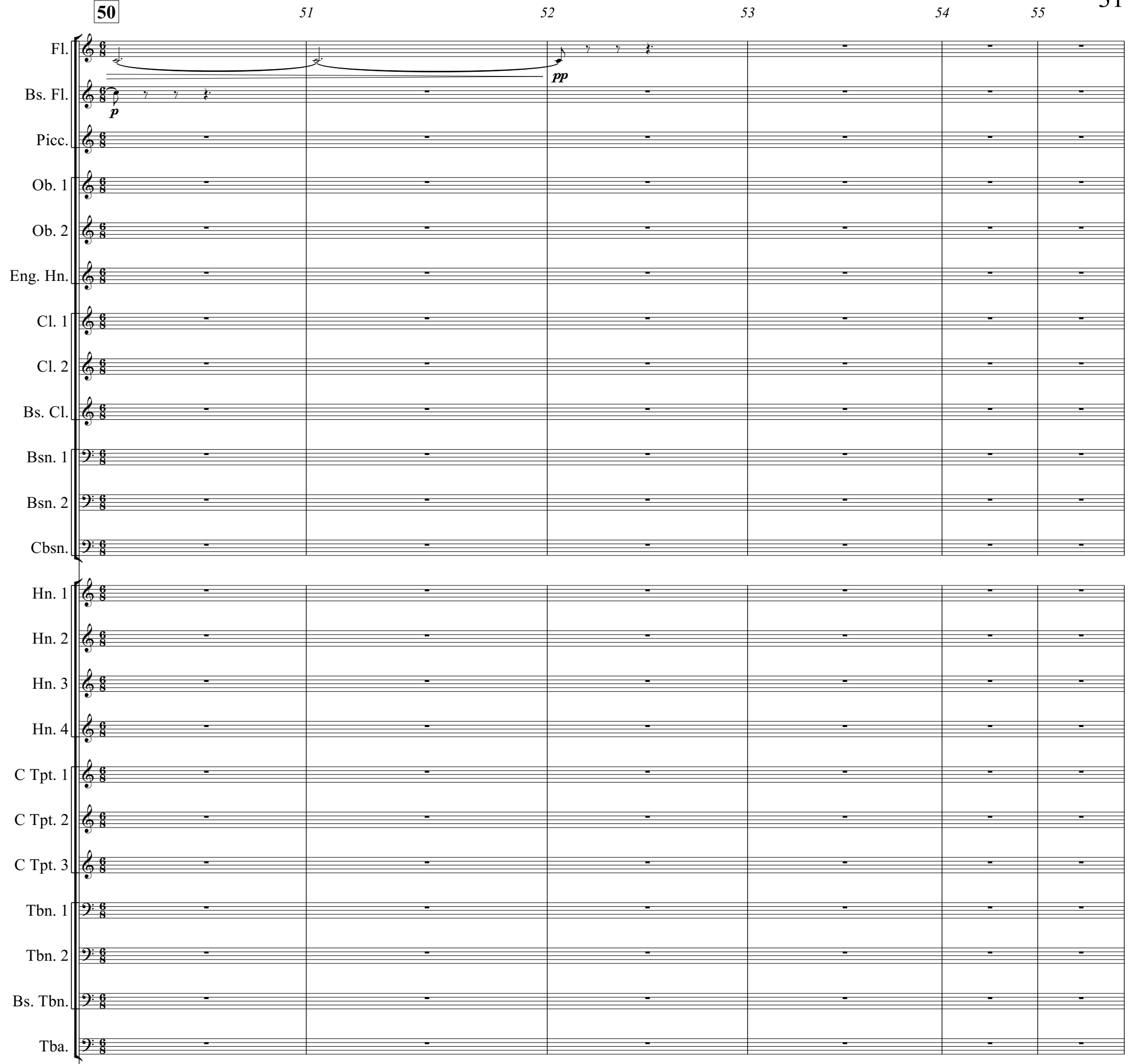

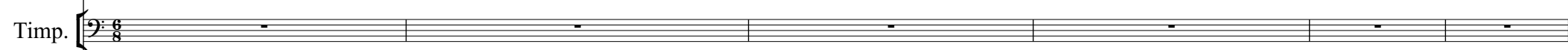
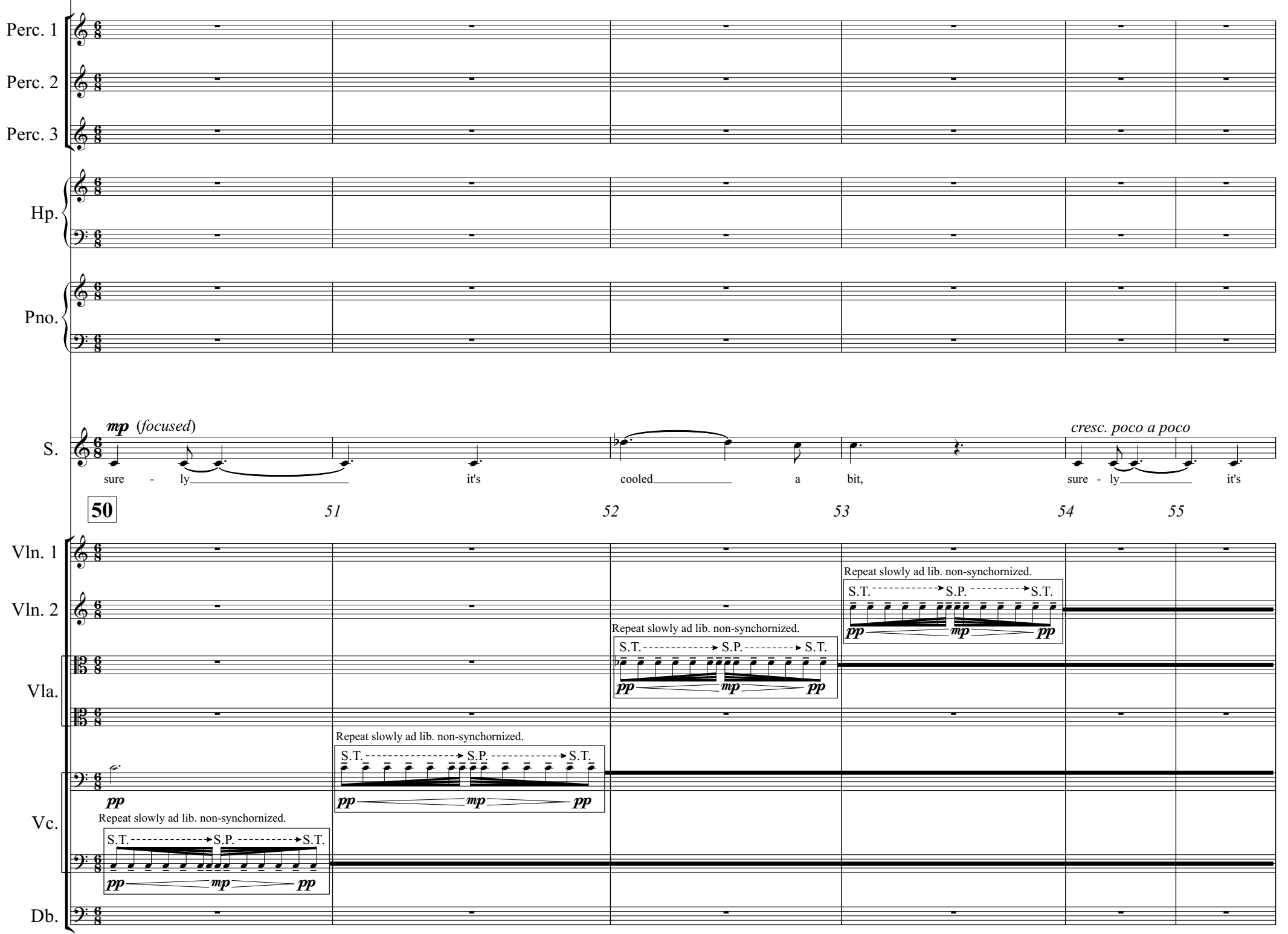

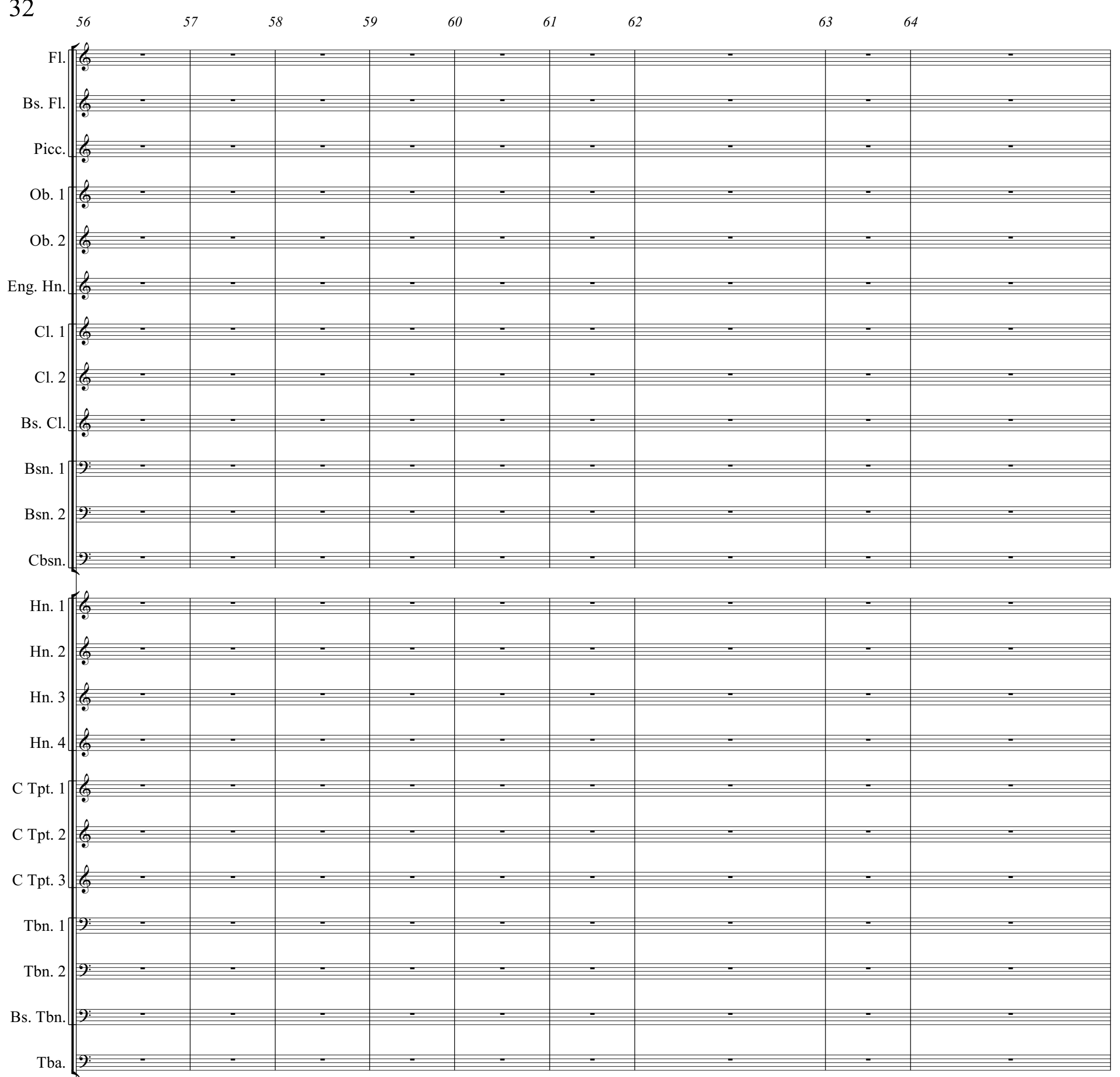

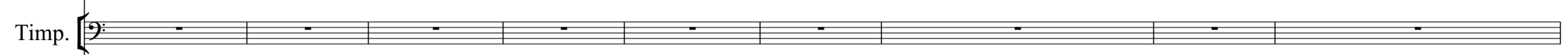

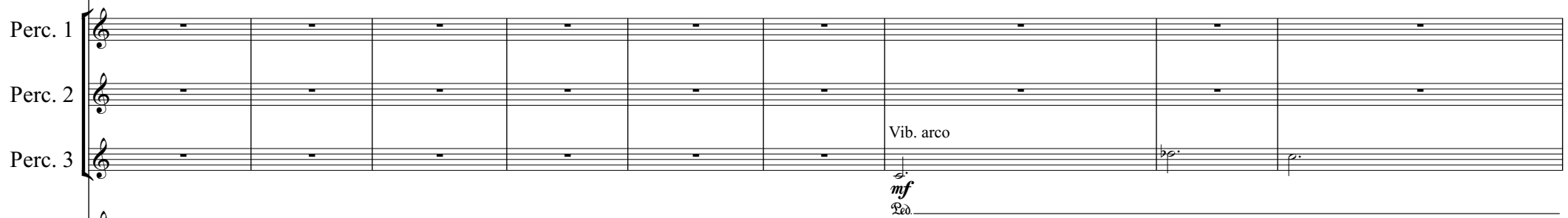

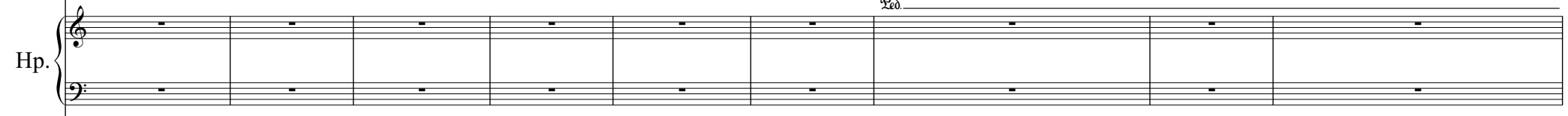

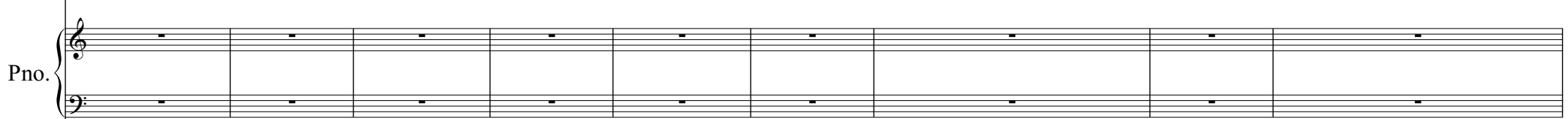

S.

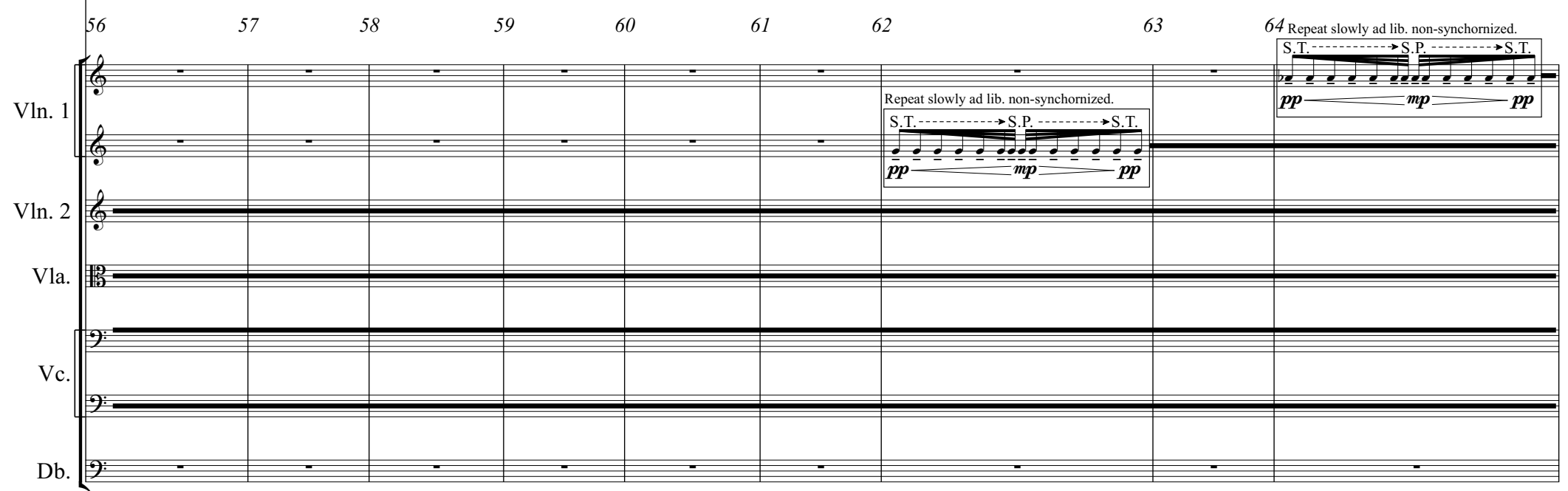




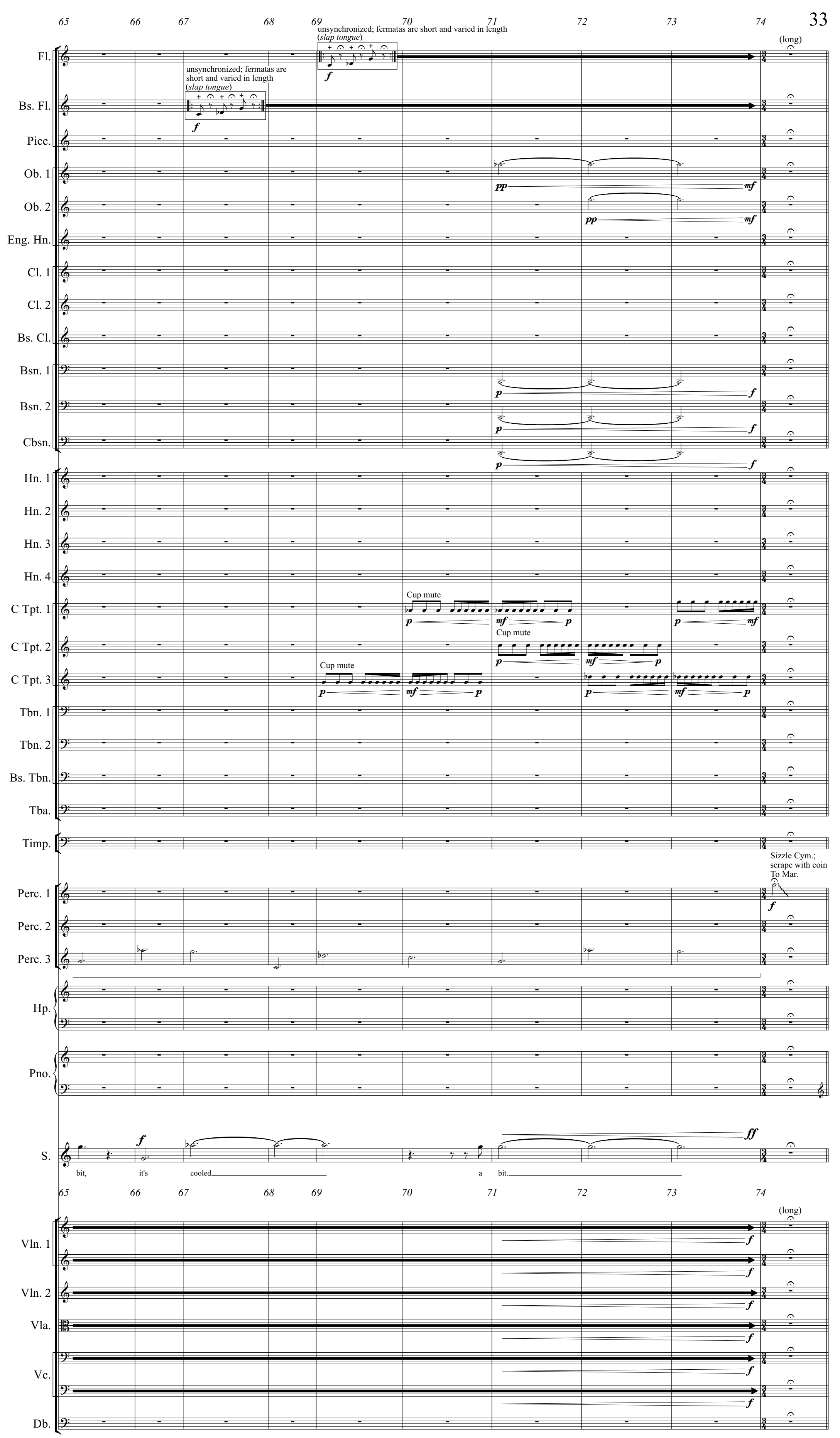




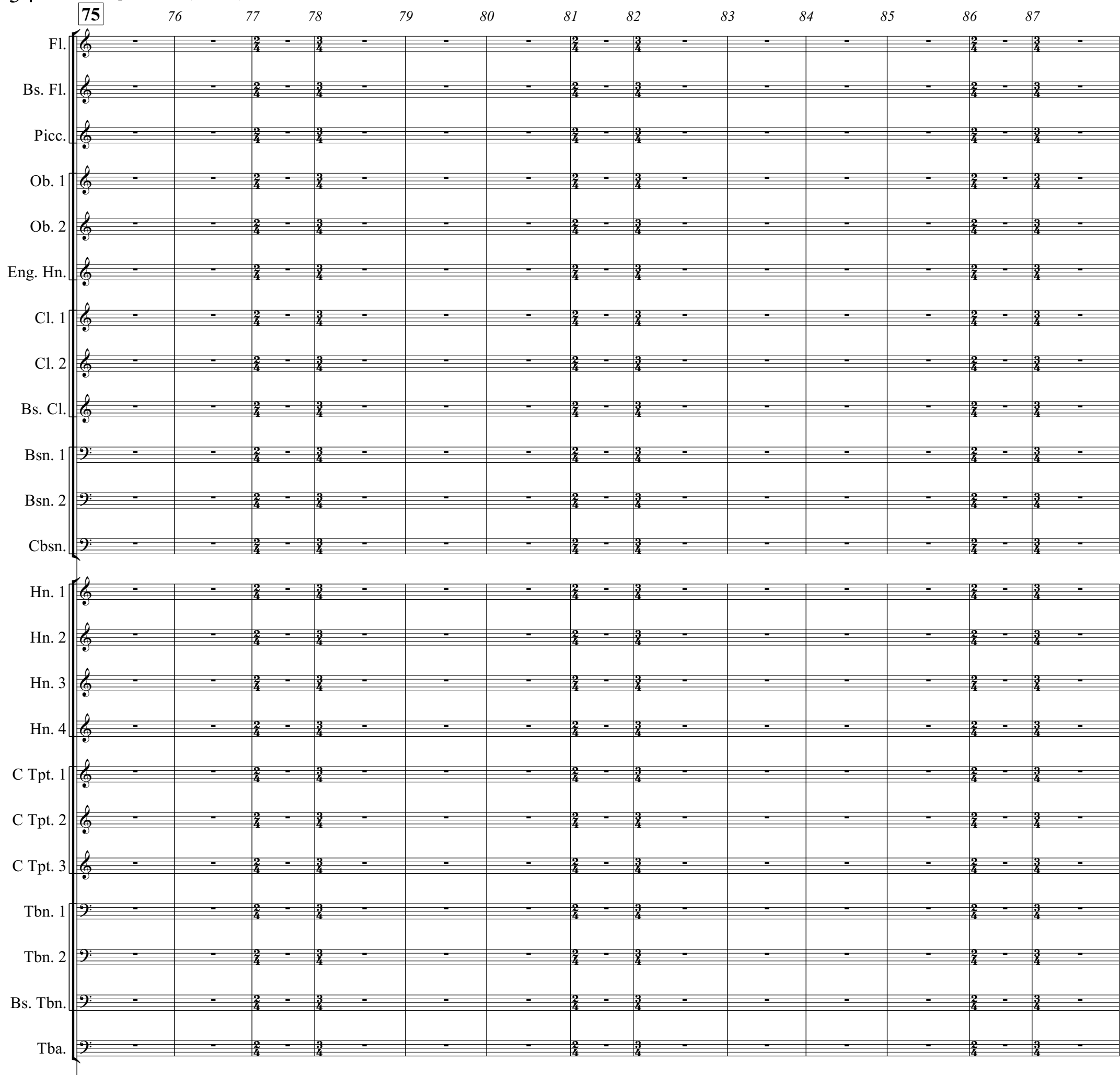

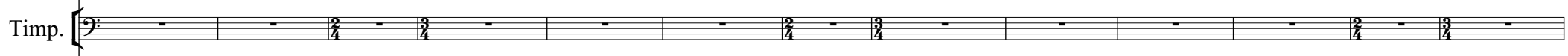

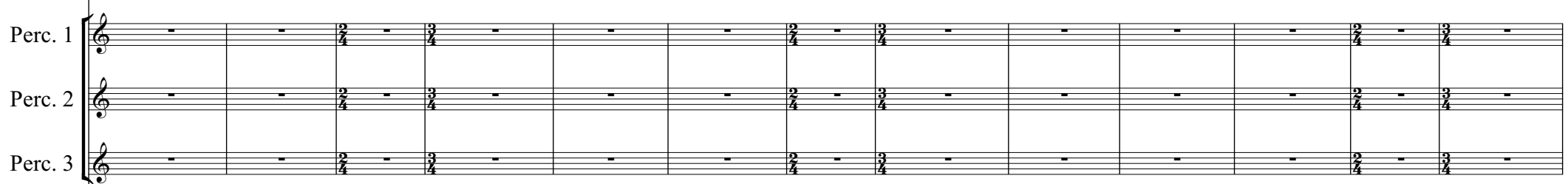

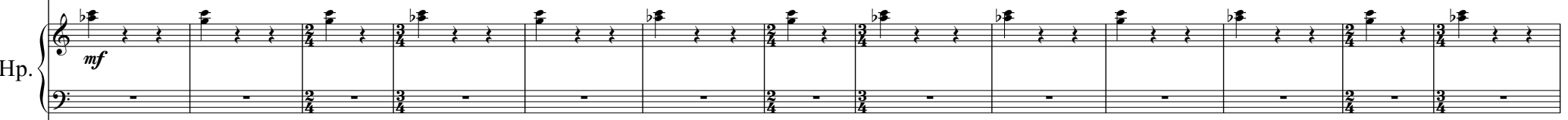

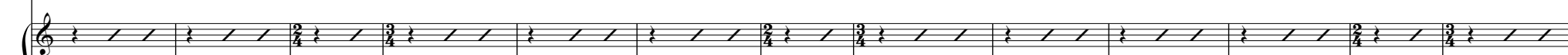

Pno. Silently depress the notes on the keyboard while struming up on
the same register of strings inside the piano with the right hand.

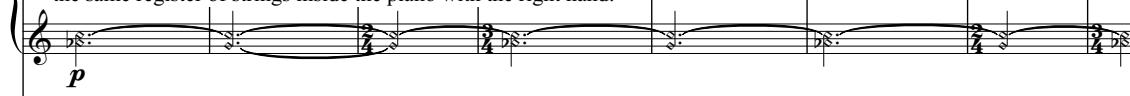

S. 75

(15 Vln.

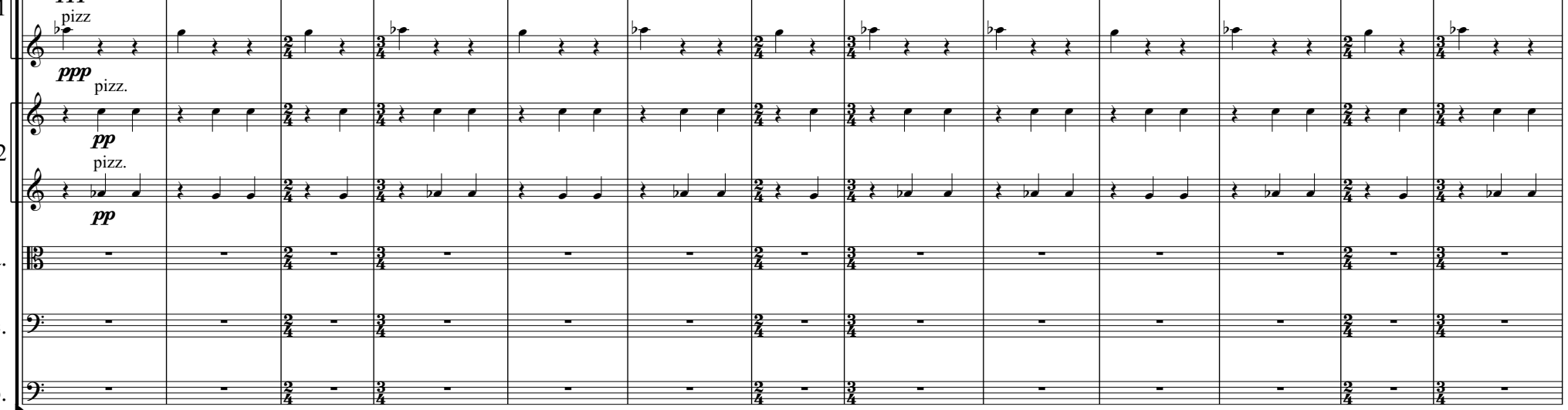



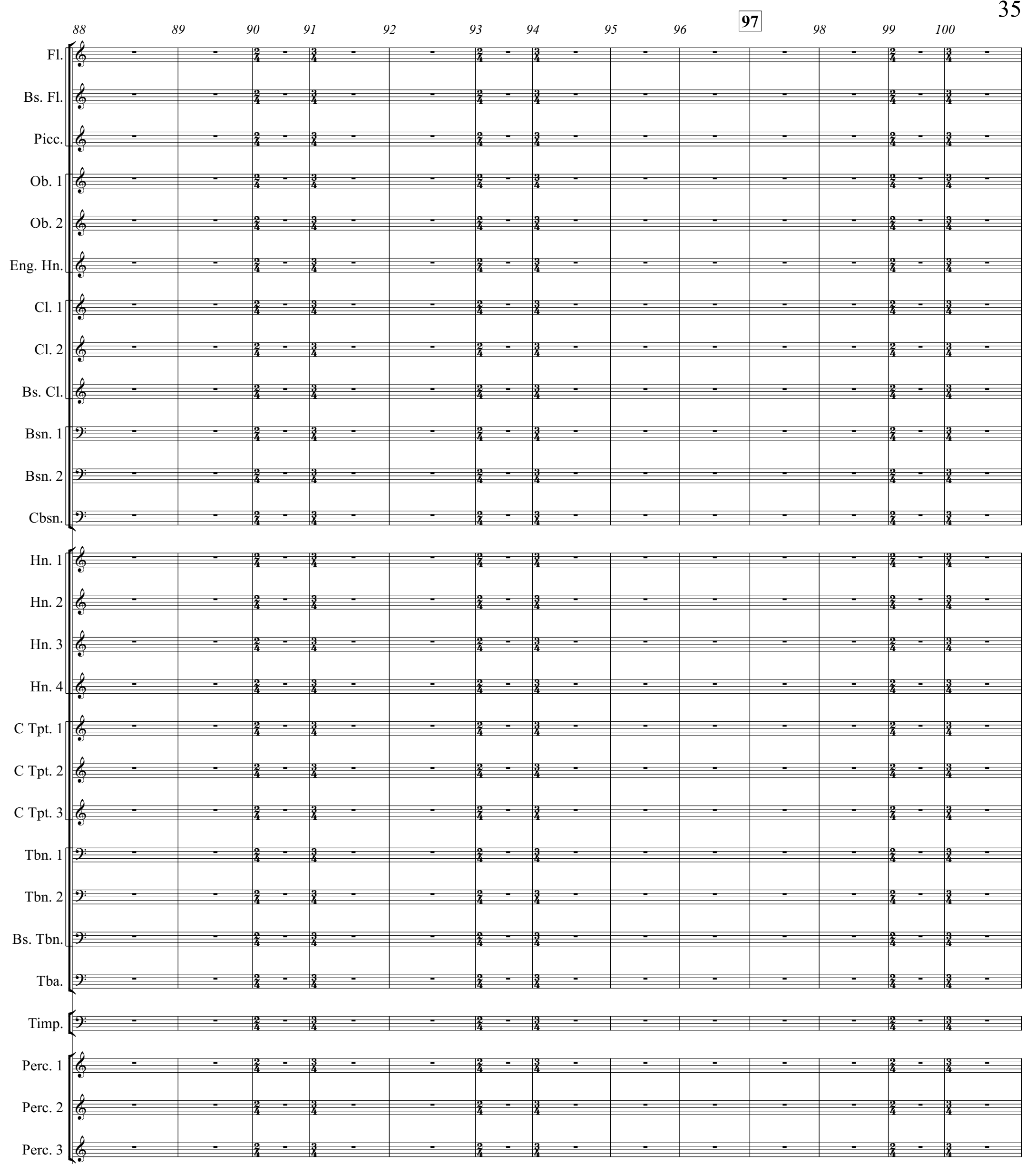

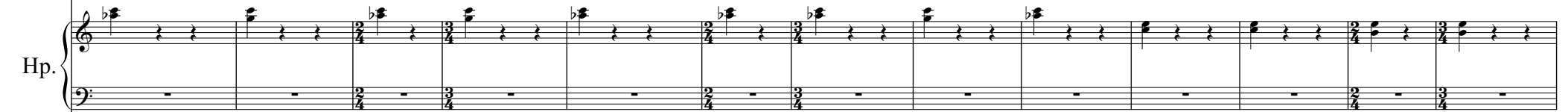

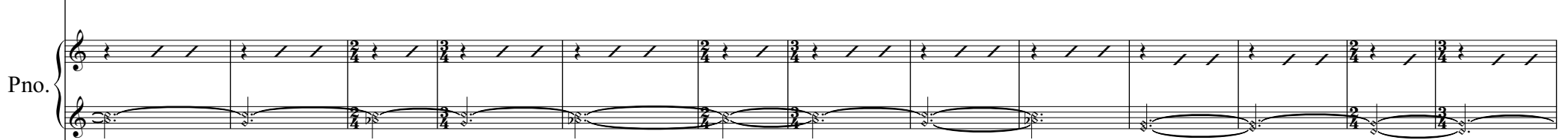

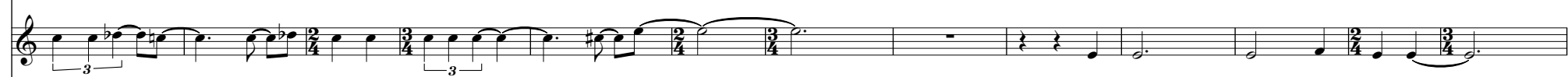

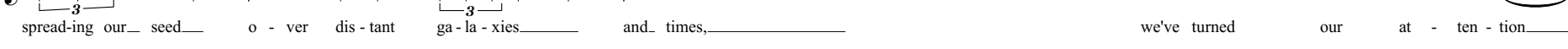
$\begin{array}{llllllllllllll}88 & 89 & 90 & 91 & 92 & 93 & 94 & 95 & 96 & 97 & 98 & 99 & 100\end{array}$

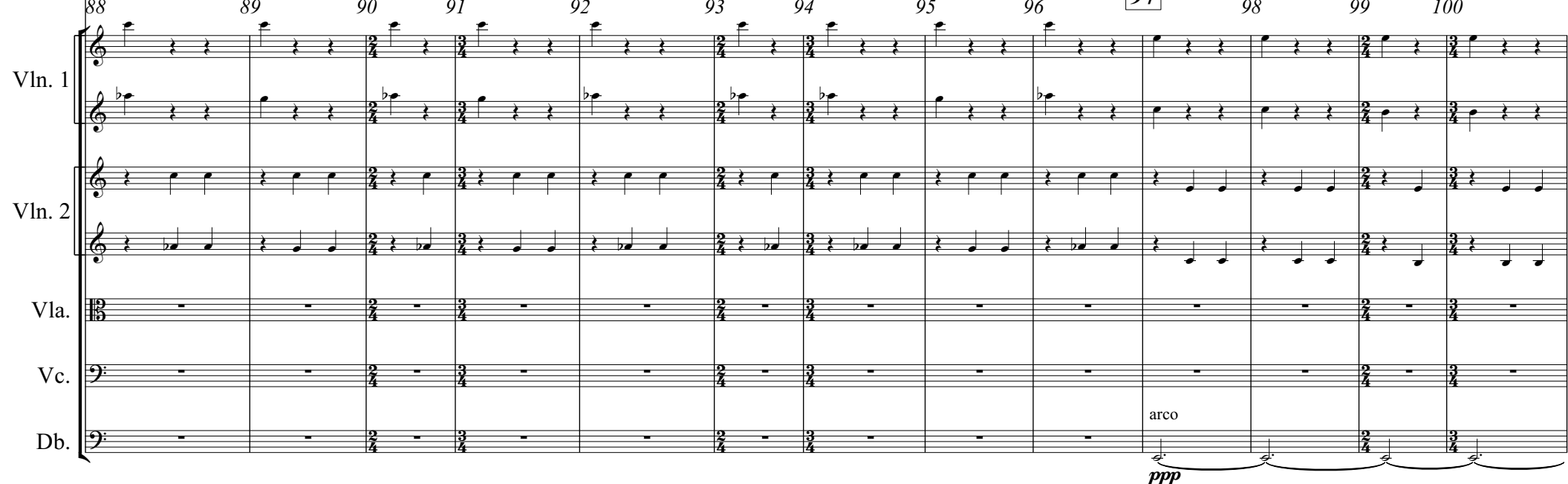



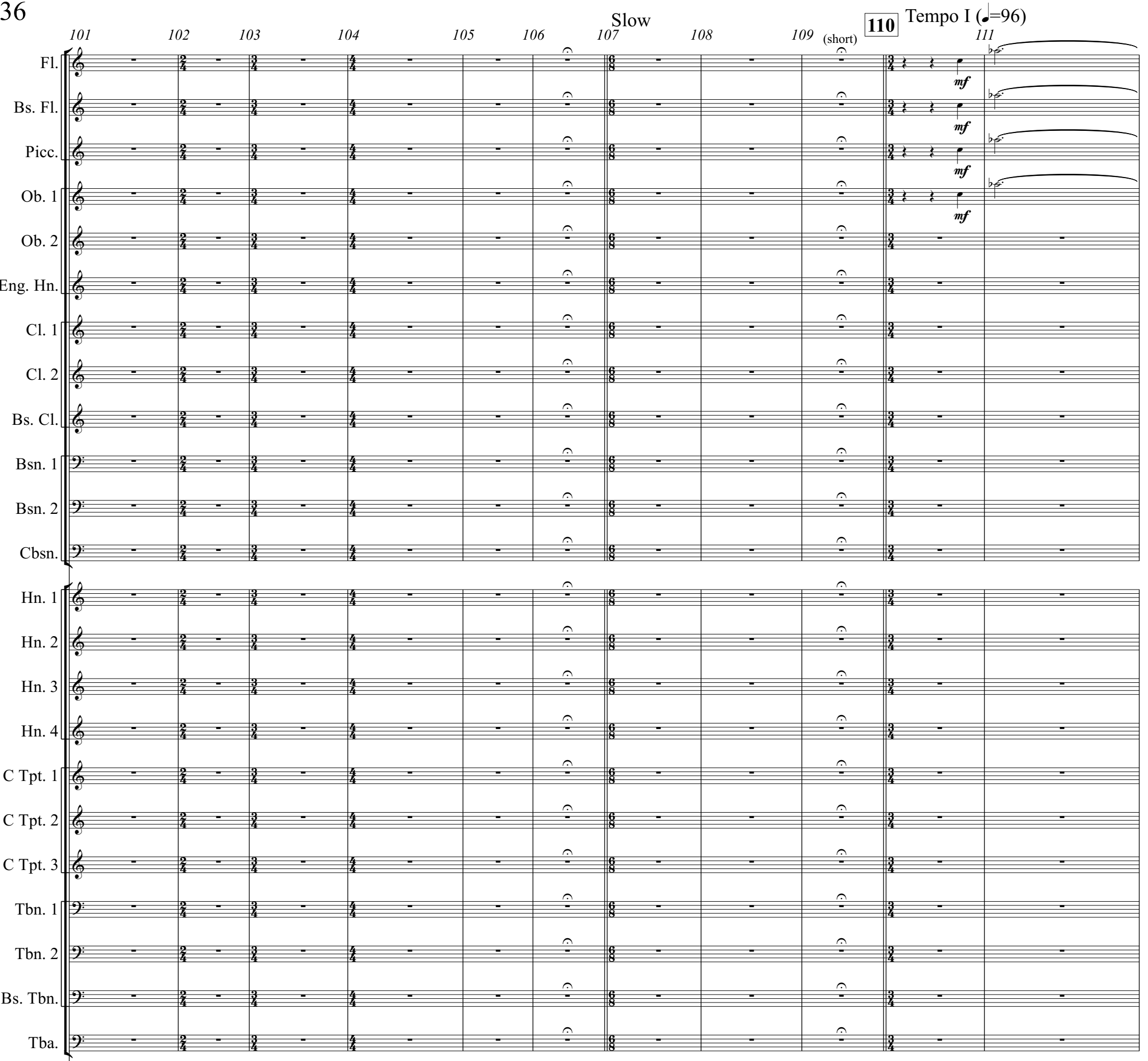

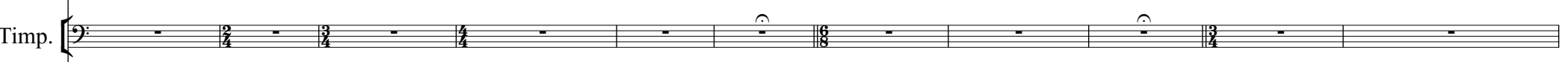
Perc. $1 \mid$\begin{tabular}{|lll|l|l|l|l|l|l|l|}
\hline \\
$P$
\end{tabular}

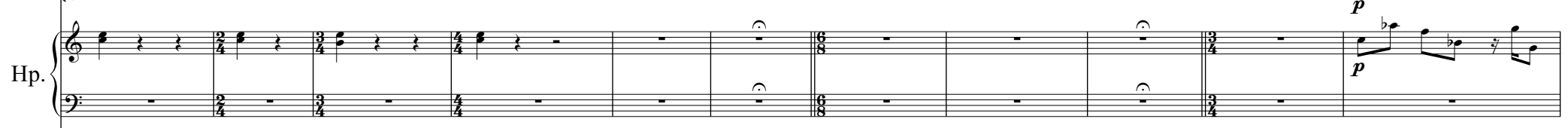

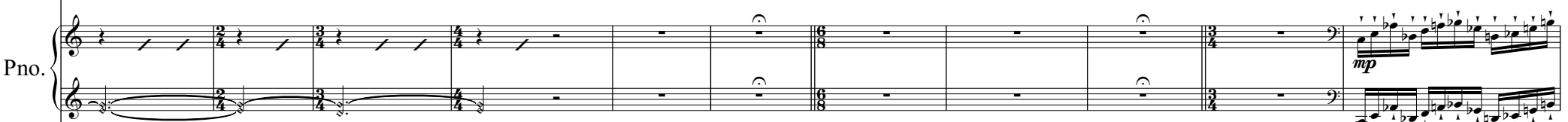

S. Vln. 2 Vln. 


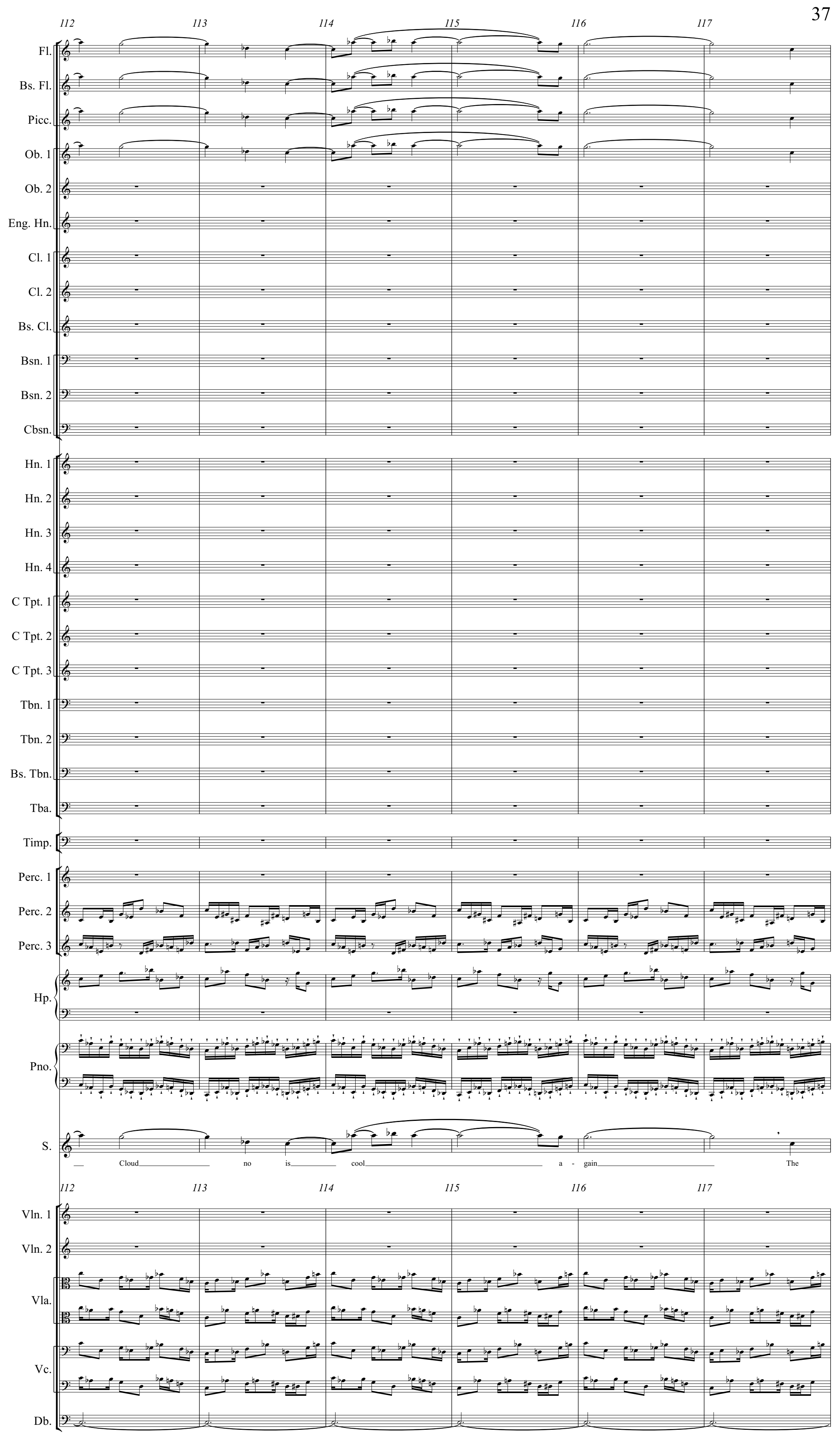



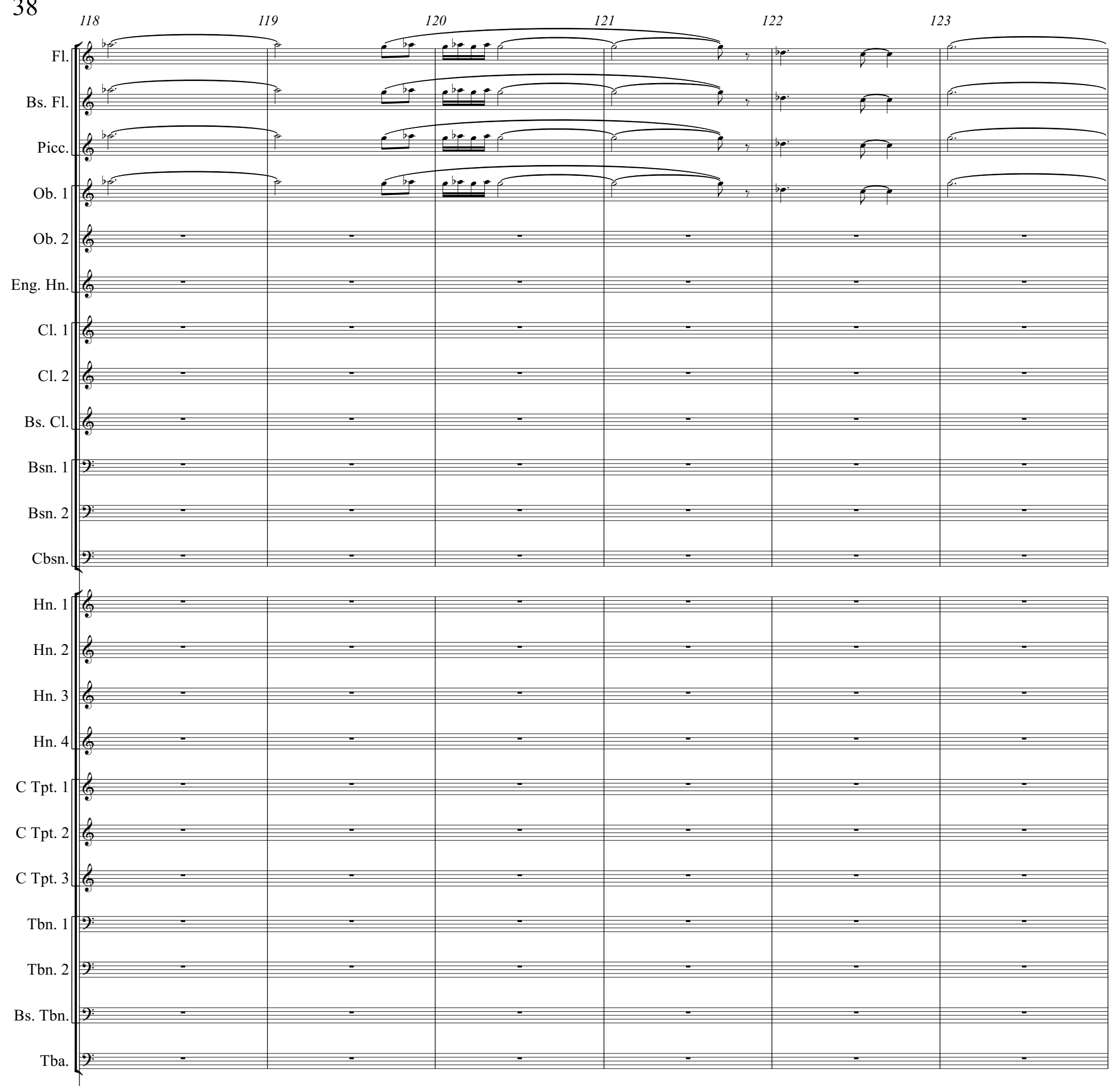

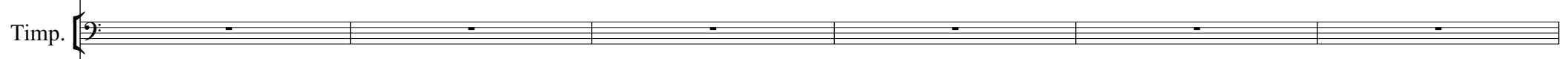

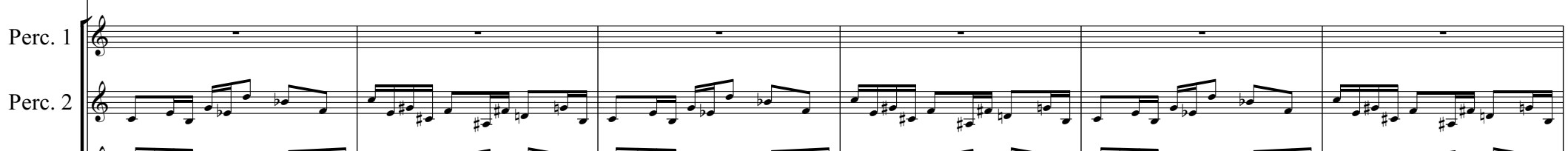

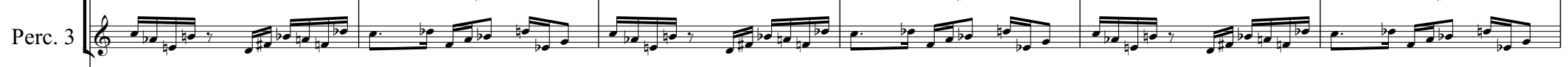

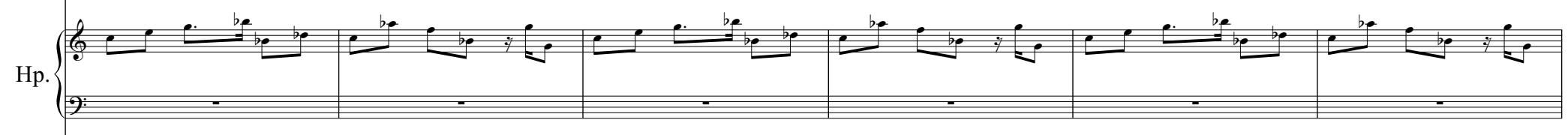
Pno. \{ VIn. 1 


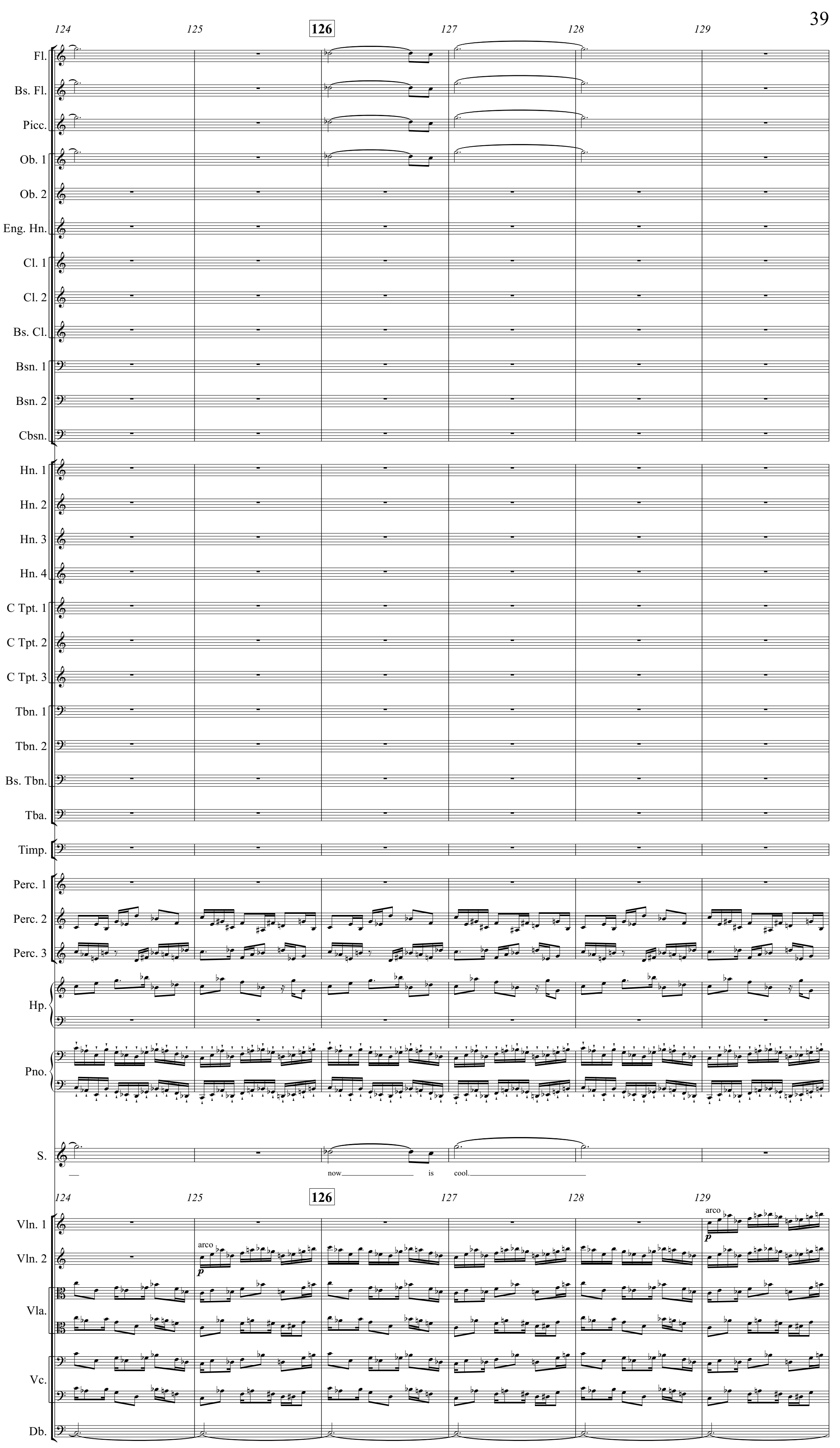


40
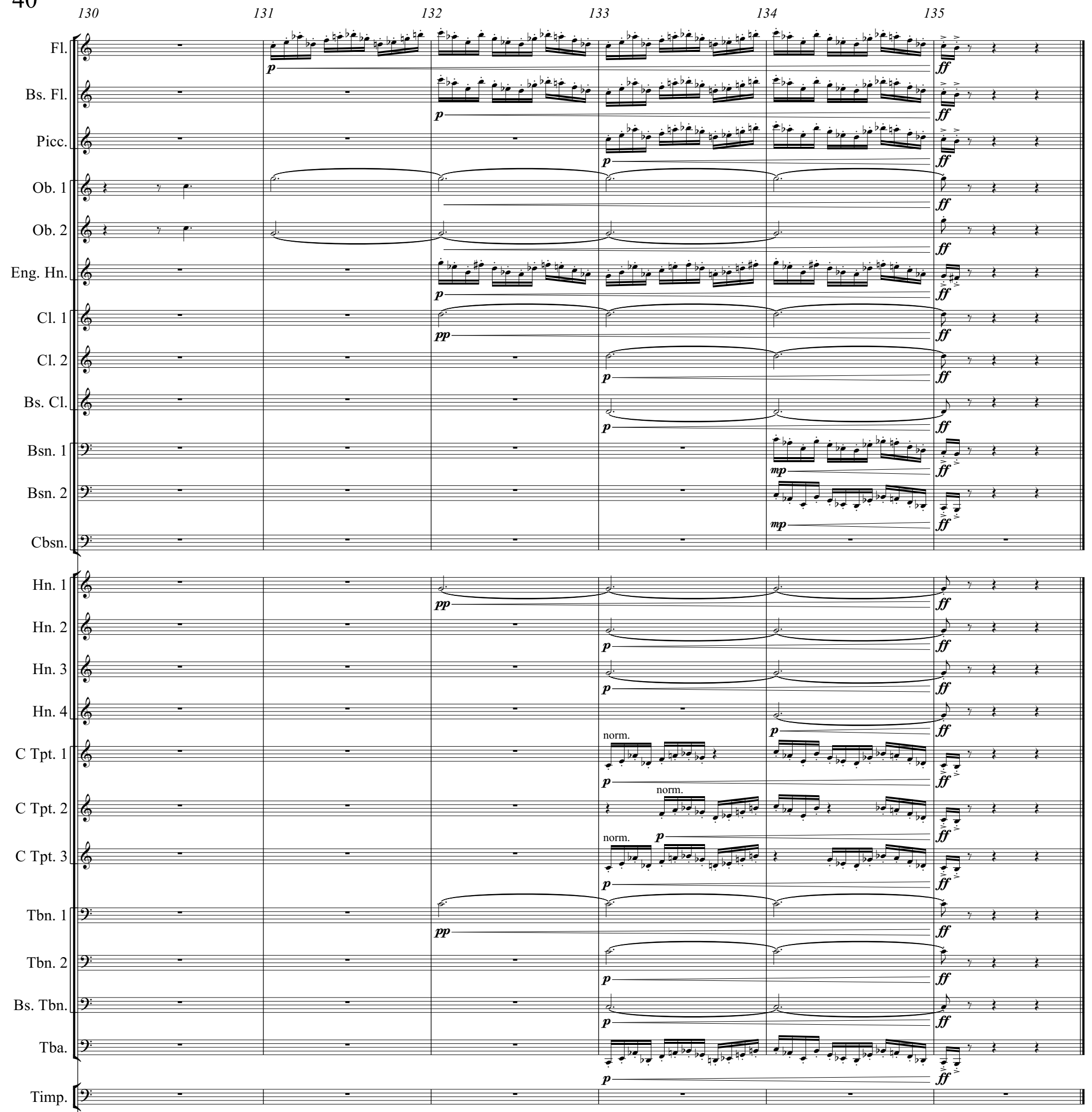
Perc. 2 Perc. 1 (1)

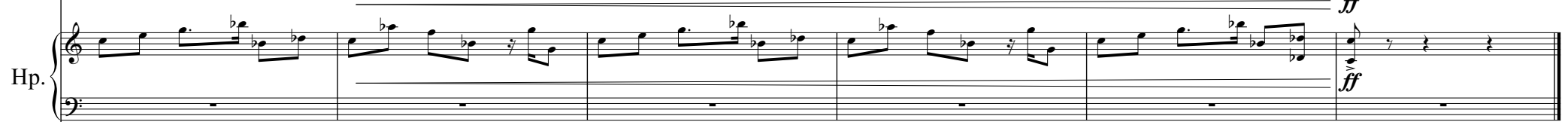
Pno. be $\begin{array}{llllll}130 & 131 & 132 & 133 & 134 & 135\end{array}$

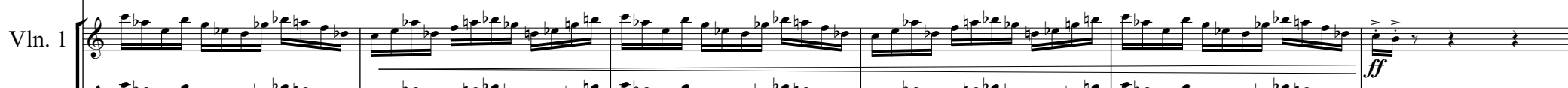

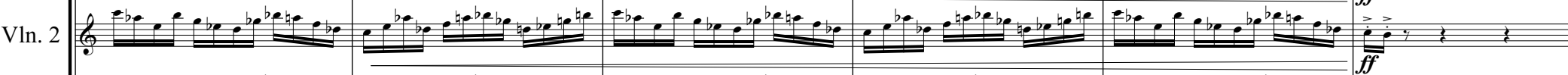
Vla. $\|_{20}$ $\mathrm{F}^{2} \mathrm{C}$

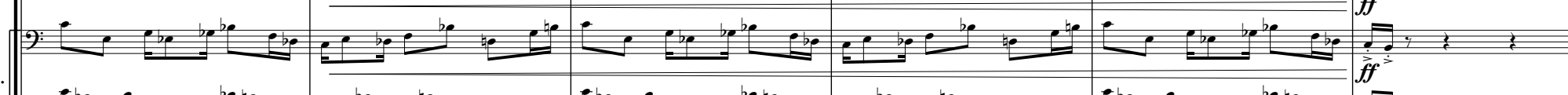
CO

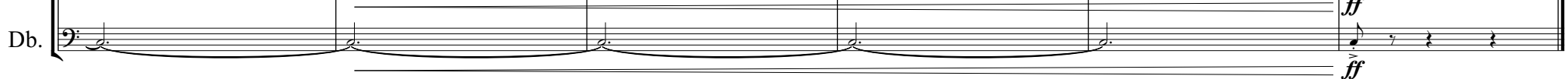




\title{
EDUCATION
}

University of Louisville, MM in composition, to be completed in 2017

- Studying composition with Dr. Steve Rouse and piano with Dr. Dror Biran and Dr. Krista Wallace-Boaz Brigham Young University - Idaho, BMA in music composition and piano, 2013

- Studied composition with Darrell Brown and Daniel Kerr, and piano with Stephen Allen

- Minored in Education and Entrepreneurship

- GPA 3.93

Clark College (WA), music composition and piano, 2010

- Studied piano with Kathy Charles, and studied composition with Ben Moll

- GPA 3.96

\section{NOTABLE COMPOSITIONS WITH PERFORMANCES}

\section{Short Cut - for orchestra (2016)}

- Premiered by the University of Louisville Orchestra at the University of Louisville's 2016 New Music Festival Duo - for oboe and piano

- Commissioned and Premiered by Dr. Jennifer Potochnic, Mar, 2017

Fairy Tale - for soprano and piano (2017)

- Premiered by Emily Johnson (sop) and Alex Isackson (pno), Mar, 2017 Nos Immortales, II. - for soprano and piano (2015-2016)

- Premiered by Emily Yocum Brown (sop) and Alex Isackson (pno), Nov. 16, 2016 Piano Concerto - for concert band with piano soloist (2014-16)

- Premiered the second movement, July 2015 by the BYU-Idaho University Band; Darrell Brown, conductor; Alex Isackson, soloist.

On a Short Leash - for electronics (2016)

- $\quad$ Premiered by the composer, March, 2016

Time Does Not Stand Still - for piano solo (2014)

- Premiered by the composer, April 2014

Cataclysmania - for concert band (2013)

- Commissioned and premiered by the Gem State Clinic Band; Darrell Brown, conductor, Feb. 2014

Chasing the J Train - for piano solo (2013)

- Premiered by the composer, Dec. 2013

The Great Conflict - cantata for tenor, baritone, and 2 pianos (2013)

- Premiered by the BYU-Idaho Opera Group, Dec. 2013

Box 'o' Chocolates - for three players (2013)

- Premiered by student performers at Brigham Young University - Idaho, 2013

- Performance by University of Louisville New Music Ensemble, April 2016 the space between light and dark - for concert band (2012)

- Premiered by the BYU-Idaho University Band, March 2013

\section{PROFESSIONAL POSITIONS AND TEACHING EXPERIENCE}

\author{
Adjunct Faculty \\ Brigham Young University - Idabo (2015) \\ - Course: -Musicianship II (Music Theory and Aural Skills) \\ - Duties: -Full responsibility for instruction and grading \\ Staff Accompanist \\ Brigham Young University - Idabo (2012-2015) \\ - Duties: -Accompany various recitals for music faculty and full-time position candidates \\ Adjudicator \\ UMTNA Composition Competition - Utah (2014-present) \\ - Judge and provide feedback for works entered by elementary to high school student composers \\ Teaching Assistant/Vocal Coach \\ Brigham Young University - Idabo (2011-2015) \\ - Course: -Group Vocal (non-music major vocal instruction) \\ - Duties: -Accompanied classes and voice labs; occasional class instruction; vocal coaching; creation of song \\ arrangements to fit students' needs
}




\section{PROFESSIONAL POSITIONS AND TEACHING EXPERIENCE - CONT.}

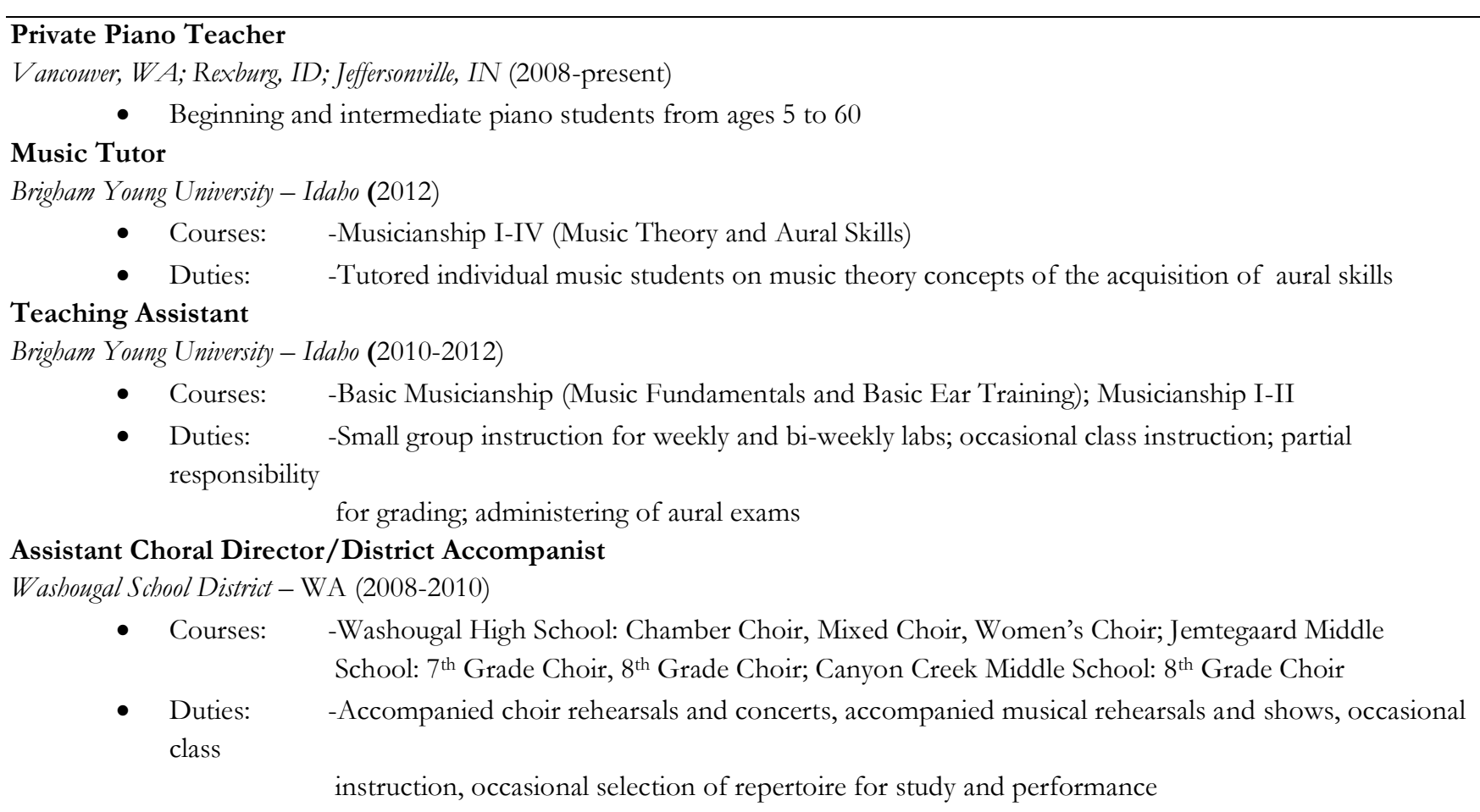

\section{PERFORMING EXPERIENCE - LARGE ENSEMBLE}

- University Wind Ensemble, University of Louisville, 2016

- University Band, Brigham Young University - Idaho, 2013-2014

- Including Soloist - Henry Cowell Concerto Piccolo for Piano and Wind Ensemble, Dec 2014

- Symphony Band, Brigham Young University - Idaho, 2014

Included CBDNA regional conference in Reno, NV

- RixStix Percussion Ensemble, Brigham Young University - Idaho, 2013

- Rexburg Tabernacle Orchestra, 2012-2013

- Piano Trio, Brigham Young University - Idaho, 2012 Included coaching from the Parisii String Quartet

- Piano Ensemble, Brigham Young University - Idaho, 2010-2011

- Symphony Band and Orchestra - Clark College, 2009-2010

- Jazz Band, Clark College, 2008-2010 Included jazz festivals in Puerto Vallarta, Mexico and Vancouver, WA

\section{PERFORMING EXPERIENCE - SMALL ENSEMBLE/ACCOMPANYING}

\section{Ensemble NjP (2015)}

- For the premier of Gene Coleman's Systole for chamber ensemble

New Music Ensemble (2015-2016)

Staff Accompanist

Brigham Young University - Idaho (2012-2015)

- Duties: -Accompany various recitals for music faculty and full-time position candidates

Private Accompanist

Brigham Young University - Idaho, Rexburg, ID; and Clark College, Vancouver, WA (2009-2015)

- Duties: -Accompany individual music students for private lessons, rehearsals, recording sessions, and performances of various types

Teaching Assistant/Vocal Coach

Brigham Young University - Idabo (2011-2015)

- Course: -Group Vocal (non-music major vocal instruction)

- Duties: -Accompanied classes and voice labs; occasional class instruction; vocal coaching; creation of song arrangements to fit students' needs

\section{AWARDS/ACHIEVEMENTS/RECOGNITION}

- Bomhard Fellowship in Music Composition, University of Louisville, 2015

- Graduate Student Dean Citation, University of Louisville, 2017

- Winner - Student Composition Competition, with She Walks in Beauty for tenor and piano, 2013.

- Featured Composer - Hymns Today Magazine, with two original hymns, May I Be In Tune and Tis On This Sweetest Day of Rest, 2013.

- Piano Scholarship - Brigham Young University - Idaho, 2011

- Piano Scholarship - Clark College, WA, 2009. 